
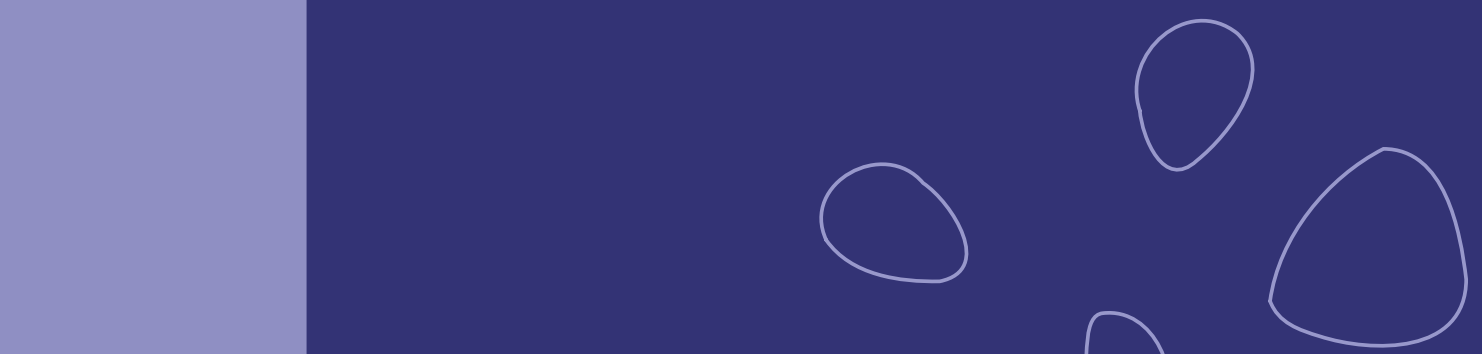

K.V. Nikolaev

Multi-dimensional analysis of nano-scale periodic structures using EUV and X-ray characterization

Theoretical concepts and applications 


\section{MUlTI-Dimensional ANALYSIS OF NANO-SCALE PERIODIC STRUCTURES USING EUV AND X-RAY CHARACTERIZATION \\ THEORETICAL CONCEPTS AND APPLICATIONS}

Konstantin Vladimirovich Nikolaev 



\title{
Multi-Dimensional ANALYSis OF NANO-SCALE PERIODIC STRUCTURES USING EUV AND X-RAY CHARACTERIZATION
}

\author{
THEORETICAL CONCEPTS AND APPLICATIONS
}

\section{DISSERTATION}

to obtain

the degree of doctor at the University of Twente, on the authority of the rector magnificus, prof.dr. T.T.M. Palstra,

on account of the decision of the Doctorate Board,

to be publicly defended

on Thursday $4^{\text {th }}$ of July 2019 at 10:45 hours

by

\section{Konstantin Vladimirovich Nikolaev}

born on the $30^{\text {th }}$ of August 1990

in Novocheboksarsk, USSR. 
This dissertation has been approved by:

Supervisor: $\quad$ prof. dr. F. Bijkerk

Co-supervisor: dr. I.A. Makhotkin

Graduation committee:

Chairman/secretary:

prof.dr. J.L. Herek University of Twente, TNW

Supervisor:

prof.dr. F. Bijkerk University of Twente, TNW

Co-supervisor:

dr. I.A. Makhotkin IMEC, Belgium

Referee:

dr. M. Gateshki

Malvern Panalytical

Members:

prof.dr. M.M.A.E. Claessens

University of Twente, TNW

prof.dr.ir J.J.W. van der Vegt

University of Twente, EWI

prof.dr. W.M.J.M. Coene

prof.dr. C. Schroer

dr.ir. I.M. Vellekoop

Delft University of Technology

Universität Hamburg, Germany

University of Twente, TNW

This work is part of the research programme of the IndustrialFocus Group XUV Optics, being part of the MESA + Institute for Nanotechnology and the University of Twente. It is supported by ASML, Carl Zeiss SMT AG and Malvern Panalytical, as well as the Province of Overijssel and the Netherlands Organization for Scientific Research (NWO).

Keywords: nano-scale structures, dynamical diffraction theory, X-ray reflectivity, extreme ultraviolet reflectivity, grazing-incidence small-angle X-ray scattering, grazing-incidence X-ray diffraction, X-ray standing waves.

Design: Cover art and artwork in the chapters headings were designed by K.V. Nikolaev. They are inspired by the numeric simulations of correlation functions, X-ray standing waves and X-ray fluorescent intensity.

ISBN: 978-90-365-4798-7

DOI: $10.3990 / 1.9789036547987$

Copyright (C) 2019 by K.V. Nikolaev. All rights reserved. No parts of this thesis may be reproduced, stored in a retrieval system or transmitted in any form or by any means without permission of the author. Alle rechten voorbehouden. Niets uit deze uitgave mag worden vermenigvuldigd, in enige vorm of op enige wijze, zonder voorafgaande schriftelijke toestemming van de auteur. 
To Natalia Ivanovna Komissarova the teacher who introduced me to physics. And to all the teachers who guided me along the way. 



\section{List of Publications}

This thesis is based on the following publications:

Chapter 2: S.N. Yakunin, I.A. Makhotkin, K.V. Nikolaev, R. W. E. van de Kruijs, M.A. Chuev, and F. Bijkerk, "Combined EUV reflectance and X-ray reflectivity data analysis of periodic multilayer structures." Opt. Express 22, 20076-20086 (2014)

Chapter 3: K.V. Nikolaev, S.N Yakunin, I.A. Makhotkin, J. de la Rie, R.V. Medvedev, A.V. Rogachev, I.M. Trunckin, A.L. Vasiliev, C.P. Hendrikx, M. Gateshki, R.W.E. van de Kruijs and F. Bijkerk, "Grazingincidence small-angle X-ray scatteringstudy of correlated lateral density fluctuations in W/Si multilayers." Acta Cryst. A75, 342-351, (2019)

Chapter 4: K.V. Nikolaev, I.A. Makhotkin, S.N. Yakunin, R.W.E. van de Kruijs, M.A. Chuev, and F. Bijkerk. "Specular reflection intensity modulated by grazing-incidence diffraction in a wide angular range." Acta Cryst. A74, 545-552, (2018).

Chapter 5: K.V. Nikolaev, V. Soltwisch, J. de la Rie, P. Hönicke, F. Scholze, R.V.E. van de Kruijs, S.N. Yakunin, I.A. Makhotkin and F. Bijkerk, "A computational scheme for the characterization of 3D nano-structures using grazing-incidence X-ray fluorescence." (to be submitted)

Chapter 6: J. de la Rie, K.V. Nikolaev, V. Soltwisch, A. Fernández-Herrero, F. Scholze, S.N. Yakunin, I.A. Makhotkin and F. Bijkerk, "EUV near field calculation for nano-scalegratings: comparison between finite element method and dynamical diffraction theory." (to be submitted) 



\section{Contents}

$\begin{array}{ll}\text { List of Publications } & 7\end{array}$

1 Introduction $\quad \mathbf{1 3}$

1.1 Motivation ........................ . . 14

1.2 Structure of this thesis . . . . . . . . . . . . . . . . 15

1.2.1 Periodical multilayer as a 1D system . . . . . . . . . . 15

1.2.2 Effect of 3D imperfections in 1D systems . . . . . . . . . . 16

1.2.3 Single-crystal surface as a 2D periodical system . . . . . . . 17

1.2.4 Artificial 3D shaped nanoscale systems . . . . . . . . . . 18

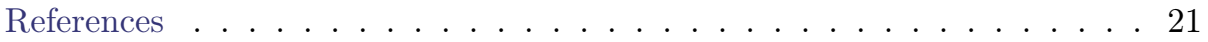

2 Combined EUV and XRR analysis $\quad 27$

2.1 Introduction. . . . . . . . . . . . . . . . . . . 28

2.2 Modeling of reflectivity from periodic multilayers. . . . . . . . . . . . 29

2.3 Parameterization of a multilayer structure . . . . . . . . . . . 30

2.4 Reconstruction and error analysis of structural parameters . . . . . . 31

2.5 Experiment layout and data processing . . . . . . . . . . . . . 32

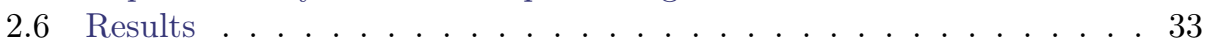

2.7 Discussion. . . . . . . . . . . . . . . . . . . 35

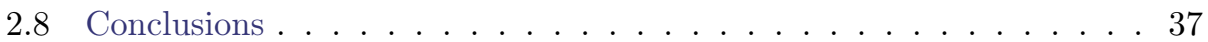

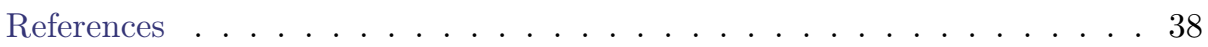

3 GISAXS study of correlated density fluctuations $\quad 41$

3.1 Introduction. . . . . . . . . . . . . . . . . . . . 42

3.2 Sample preparation and characterization . . . . . . . . . . . . . 42

3.2.1 Sample preparation. . . . . . . . . . . . . . . . 42

3.2.2 Preliminary sample characterization

with XRR and HAADF-STEM . . . . . . . . . . . . . . 42

3.2.3 GISAXS experiment . . . . . . . . . . . . . . . . . . . . . . . 44

3.3 GISAXS theoretical background . . . . . . . . . . . . . 45

3.3.1 Scattering on correlated interface roughness . . . . . . . . . 46

3.3.2 Scattering on the density fluctuations . . . . . . . . . . . 46

3.4 Results and discussion . . . . . . . . . . . . . . . . . . . . 47

3.4.1 Analysis of correlated interface roughness . . . . . . . . . 47

3.4.2 Analysis of correlated density fluctuations . . . . . . . . . . 48

3.5 Conclusions...................... . . 51

3.6 Appendix ........................ 51

3.6.A Correlation function of the para-crystal model . . . . . . . . 51

3.6.B Variation of statistical parameters ... . . . . . . . . 53

3.6.C Statistical analysis of HAADF-STEM image . . . . . . . . 56 
3.6.D XRR analysis . . . . . . . . . . . . . . . 57

References ..................... . . 60

4 Specular-reflection intensity modulated by GID $\quad 63$

4.1 Introduction. . . . . . . . . . . . . . . . . . . 64

4.2 Theoretical background . . . . . . . . . . . . . . . . 65

4.3 Geometry of the diffraction of evanescent X-rays. . . . . . . . . . . . 67

4.4 Diffraction of an evanescent wave . . . . . . . . . . . . . . 68

4.5 Conclusions . . . . . . . . . . . . . . . . . . 73

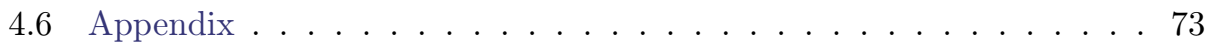

4.6.A Numerical simulation of the dispersion surface . . . . . . . . 73

4.6.B Comparison of simulations that use three roots and four roots matrices .................. . 74

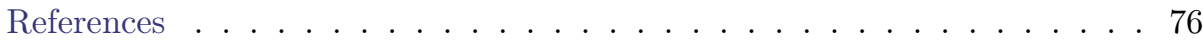

$5 \quad 3 \mathrm{D}$ X-ray standing waves $\quad \mathbf{7 9}$

5.1 Introduction. . . . . . . . . . . . . . . . . . 80

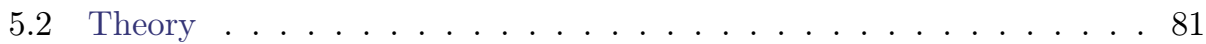

5.2.1 Many beam dynamical diffraction theory . . . . . . . . . . . 81

5.2 .2 Characteristic equation ................... 83

5.2 .3 Boundary conditions . . . . . . . . . . . . . . . 84

5.2 .4 Numerical stability . . . . . . . . . . . . . . . . . 84

5.2.5 X-ray fluorescence intensity . . . . . . . . . . . . . . . 86

5.3 Numerical simulations . . . . . . . . . . . . . . . . . . . 87

5.3.1 2D structure: $\mathrm{Si}_{3} \mathrm{~N}_{4}$ lamelar grating . . . . . . . . . . 88

5.3.2 3D structure: Cr nano-columns . . . . . . . . . . . . 90

5.4 Discussion. . . . . . . . . . . . . . . . . . . 94

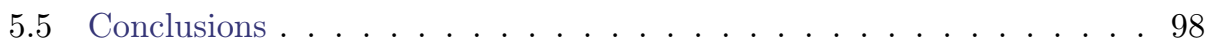

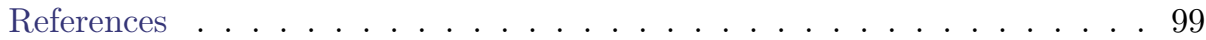

6 EUV near field calculation for nano-scale gratings 103

6.1 Introduction. . . . . . . . . . . . . . . . . . . 104

6.2 Numerical simulations . . . . . . . . . . . . . . . . . . . . . . . 105

6.2.1 Dispersion surface . . . . . . . . . . . . . . . 108

6.2.2 Comparison to scalar formulation . . . . . . . . . . . 109

6.3 Conclusions . . . . . . . . . . . . . . . . . . . . 111

6.4 Appendix ............................ 111

6.4.A The parametrization of test samples and geometry of the simulations . . . . . . . . . . . . . . . . 111

6.4.B Dynamical solution to the wave equation . . . . . . . . . . 112

6.4.C Boundary conditions . . . . . . . . . . . . . . 115

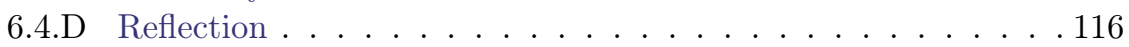

6.4.E Near field . . . . . . . . . . . . . . . . . . . . . . . . . . . . . . . . . .

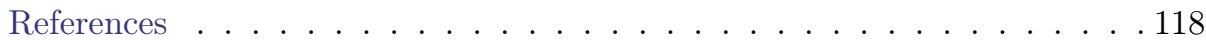

$\begin{array}{ll}\text { Valorization and outlook } & 121\end{array}$

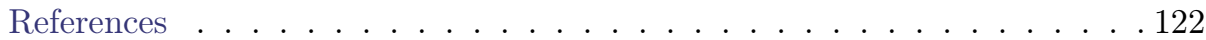


Summary 123

Samenvatting 125

Заключение 127

Acknowledgments 129 



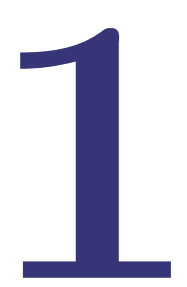

\section{INTRODUCTION}




\subsection{Motivation}

There is a long way from the initial discovery of physical phenomena until they can be routinely used in science laboratories and in industrial processes. In that regard, X-ray metrology is not an exception. Starting with its discovery in 1913, X-ray diffraction on crystals [1] has been used as a unique tool for fundamental research in solid state physics and quantum physics, among other things. The use of very short probe wavelengths, like X-ray radiation, has revealed materials properties with extreme resolution. However, the effects of this discovery have only become tangible in everyday life after half century. This was when Rietveld developed a mathematical model for the analysis of X-ray powder diffraction, the Rietveld refinement algorythm [2], which allowed the analysis of the crystalline structure of complex compounded materials. Consequently, this boosted developments in many different fields of industry like energy, semiconductor, pharmaceutical and medical industries (see examples in [3-6])

A similar tool for analytical research is X-ray reflectivity. Its potential for the characterization of thin-film structures was demonstrated by Kiessig in a landmark experiment [7] in 1933. Later, in 1954 Parratt derived equations to account for interference, refraction and absorption processes in complex multilayered structures with layer thicknesses down to sub-tenth nanometers [8]. This allowed the use of $\mathrm{X}$-ray reflectivity for structural analysis of multilayers, and found its application in the development of lasers [9], instruments for astronomy observations [10], and in the fabrication of integrated circuits elements [11]. Initially, the technological applications were focused on the internal, nm-thin subsurface structure of the sample and were later also aimed at the characterization of the internal structure of crystals and thin film multilayered structures.

Currently applied X-ray analytical methods have an extremely broad spectrum of applications, both in fundamental and in industrially driven research. An exemple of the first is the recent ultra-fast Einstein-de Haas effect study, which used X-ray diffraction [12]. This effect consists of conversion of the magnetic moment into mechanical angular momentum during rapid demagnetization of certain types of ferromagnets. This was predicted and experimentally observed in 1996 [13], although the physical process involved in the momentum transfer remained unknown until recently. In [12], surface X-ray diffraction has been used to characterize this process. It revealed that such momentum transfer involves vibration of the crystal lattice on an ultra-fast time scale. This is a vivid example of the use of X-ray diffraction for fundamental research. In parallel, this high resolution X-ray diffraction is routinely applied in industrially driven research. For example, it is used for the analysis of thin epitaxial films employed in LEDs [14]. X-ray reflectivity is applied for the characterization of the thickness of optical coatings [15] and various metallic coatings [16] in semiconductor devices. X-ray scattering can be routinely applied by pharmaceutical industry for the characterization of nano-particles [17], colloid solutions and protein shapes [18]. Recent developments of nano-surface patterning and nano-fabrication have demonstrated unique capabilities of surface based devices, for example topological insulators [19], spintronics [20] and superconducting computing[21]. 
Although many techniques are considered to be fully developed, in practice their direct application to objects developed for cutting edge technologies often has room for improvement. An example is the manufacturing of periodic multilayer structures for short wavelength optics. Interest in studies on such 'mirrors' is driven by the fact that the performance of these devices is very sensitive to interface imperfections. The requirements of the modern industry necessitates a reduction of the thin film dimensions, down to the level where the characteristic depth of the interface imperfection is comparable to the thickness of the layer itself. This raises two main questions. Firstly, how does mutual diffusion affect the optical parameters of each layer in the device? Conventional X-ray reflectivity analysis is sensitive to the diffusion depth, but does not have sensitivity to the elemental composition of each layer. This may lead to incorrect conclusions on the optical properties of multilayer mirrors, for instance in the Extreme UV range. Secondly, can interface roughness in such thin film systems be affected by the bulk imperfections of the layer itself? Grazing-incidence small-angle X-ray scattering is commonly applied to the study of interface roughness. However, this technique does not consider bulk imperfections and interface roughness simultaneously. In addition to the example of multilayer structures other examples can be given such as 2D and 3D periodically patterned nano-scale structures. Among other structural parameters, the performance of such devices depends on their structure along the lateral direction. Recently, a state of the art experimental scheme [22, 23], based on the X-ray standing wave technique, has been demonstrated for an element-selective study of the atomic distribution along the lateral plane as well as in-depth. However, novel computational schemes were found to be required for the analysis of this experimental data [23]. Many scientific challenges of using X-ray diffraction and X-ray reflectivity can be addressed by using lab-scale X-ray tools, while grazing-incidence small-angle X-ray scattering, surface X-ray diffraction typically require higher brilliance, but less accessible sources like synchrotron light sources or free-electron lasers. As we discussed above, a broad availability of metrological tools is of high importance for industry.

The above depicted state of short-wavelength metrology justifies a further, specific metrological development. In this thesis we revisit several known techniques in order to extend their applicability for the characterization of ultra-thin multilayers, single-crystal surfaces and 3D patterned nanoscale devices. The work covered in this thesis is of a theoretical character with many cases demonstrated by experimental verifications. Our effort is focused on modern nano-metrology characterization techniques with evidence of improvements by better understanding of the underlaying physics. In the following section we will discuss the problems posed above and ways to solve them in detail.

\subsection{Structure of this thesis}

\subsubsection{Periodical multilayer as a 1D system}

X-ray reflectivity (XRR) measurements are widely used in the physics of thin films [24]. They allow in-depth analysis of optical properties of thin film single- and multilayer structures. More specifically, this implies solving the inverse problem of 
$\mathrm{X}$-ray reflectivity of the multilayer systems, i.e. one can reconstruct the depth profile of the refractive index that represents the atomic distribution in the multilayer. In that regard a multilayer is basically a $1 \mathrm{D}$ structure since the optical properties of a multilayer vary only with the depth in the layered stack.

However, different multilayer systems with different chemical compounds and/or stoichiometry can have a similar profile. This ambiguity might be problematic when the XRR data is used to predict optical properties of the structure in a different spectral range. This is especially important in the EUV spectral range since photon interaction with matter is stronger here than in the X-ray range [18]. This problem is dealt with in Chapter 2; we simultaneously analyze reflectivity curves measured at different photon energy, namely in the X-ray and EUV range. We use the covariation analysis based on the so-called inverse Hessian matrix [25] to estimate the stoichiometry values of the multilayer and their uncertainties. The error analysis showed that simultaneous fitting of the X-ray and EUV reflectivity data reduces errors in the stoichiometry. This can be explained by different absorption coefficients in the X-ray and EUV ranges, consequently reducing the cross-correlation between parameters of the system. Covariation analysis confirms this assumption. This allowed us to simulate optical properties of the multilayer correctly (in accordance to the experiment) in the EUV regime. Moreover, covariation analysis allowed us to separately investigate contribution of different sets of data to verify the correctness of the characterization of the multilayer structure.

\subsubsection{Effect of 3D imperfections in 1D systems}

As discussed above, optical properties of thin film multilayers are strongly dependent on their 1D structure. This approximation is valid when the lateral fluctuations of interface roughness or layer densities can be neglected. However, in samples with large interface roughness or contamination inclusions in the form of nanoparticles, part of the incident beam elastically scatters from these imperfections causing diffuse scattering. Generally, these imperfections have a 3D character and thus diffusely scattered photons can scatter off-specularly. One can think of diffuse scattering as a sub-specular reflection event i.e. a specular reflection from the effective interface of the imperfection. Consequently, emerging patterns in the spatial distribution of defects leads to patterns in the distribution of diffuse scattering intensity along different scattering directions. Small-angle X-ray scattering is a technique which consists of a set of theoretical and experimental tools for the study of the morphology of structural imperfections. In theoretical physics such characterization tasks are assessed using the perturbation theory [26].

The perturbation theory is used for finding approximate solutions for systems in which only a part can be solved exactly while system as a whole lacking exact solution. Take for instance an example of the motion of our planet around the sun. Our planet deviates slightly form its Kepler orbit, being attracted by other planets in the solar system. Amplitude of such deviations can be calculated by using the perturbation thory, where only the interaction of our planet only with the sun yields an exact solution (Kepler orbit) and the complex, yet weak, attraction to other planets is considered as perturbation. Analogously, in our case a multilayer model 
is composed of two parts: a perfect $1 \mathrm{D}$ multilayer system and a $3 \mathrm{D}$ distribution of structural imperfections. Propagation through the perfect multilayer is calculated using the Parratt formalism and structural imperfections are considered as a source of diffusely-scattered photons. Sequentially, their propagation is considered as a propagation in an ideal multilayer. The quality of such approximation is considered good when the characteristic sizes of the imperfections are lower than those of an ideal structure [27].

In Chapter 3 we study a multilayer system using Grazing-Incidence Small-Angle X-ray scattering (GISAXS). We found that off-specular scattering from our W/Si multilayer, prepared by magnetron sputtering deposition, contains a pattern that could not be described in conventional GISAXS theory simulating scattering at interface roughness. Subsequent characterization of our multilayers with scanning transmission electron microscopy revealed peculiar density fluctuations within the Si layers. To investigate these, and to confirm STEM observation, GISAXS is used. To the best of our knowledge such density fluctuations in multilayer systems were not covered by GISAXS study before. Therefore we build a mathematical model of diffuse X-ray scattering on density fluctuations, based on the para-crystal model [28, 29]: a model in which defects are considered to be distributed similarly to atoms in a crystal that have a short range order but lack a long range order.

The study in Chapter 3 covers effects appearing in diffuse X-ray scattering due to density fluctuations, and how these effects are distinguished from those resulting from interface roughness. We also discuss how diffuse scattering changes with a change of parameters of density fluctuations, based on mathematical simulations. Independently, Maruyama et.al. found similar density fluctuations in Ge-containing multilayers [30], emphasizing the relevance of such research.

\subsubsection{Single-crystal surface as a $2 \mathrm{D}$ periodical system}

In the previous sections we considered specular reflection and diffuse scattering of $\mathrm{X}$-rays from multilayer structures. In most of the practical cases, specular reflection intensity is much higher than the intensity of diffuse scattering at grazing incidence (see Eqs. 7-9 in [31]). Therefore, the effect of interference between specularly reflected photons and diffusely scattered photons on the specular reflection itself is negligible. Generally speaking, the reverse is not true: interference between reflected and scattered photons give rise to resonant Bragg sheets in diffuse scattering intensity distribution [32-34]. But what if the intensities of the reflected and scattered photons are of the same order of magnitude? Such a scattering mechanism can be observed in grazing incidence diffraction (GID) experiments [35, 36].

In the geometry of GID, the incident beam and the sample are aligned such that the angle of incidence is between $\theta_{c}$ and $2 \theta_{c}$, with $\theta_{c}$ being the critical angle, while the sample is azimuthally rotated to meet the Bragg conditions for crystal lattice planes perpendicular to the surface of the sample. In the publications of Bushuev et.al. [37-39] it is shown that in these conditions the specular reflection intensity is modulated by the diffraction near the Bragg conditions. These modulations are extremely sensitive to the structure of the surface of the crystal. In that regard one can consider the surface sample as a $2 \mathrm{D}$ system where the change of the lateral 
crystal structure is taken into account as a function of its depth.

Using the dynamical diffraction theory with a two-beam approximation in Chapter 4 , we predict an interesting effect. For the samples with modified sub-structure (for example due to the interaction with oxygen in atmosphere or a nm-thick epitaxial layer on top of a single-crystal) we predict specular reflectivity modulations in a wide angular range far from the Bragg conditions. One can think of this effect as an analogy to an acoustic beat when two acoustic waves with a slight difference in phase interfere, creating time-dependent modulations in the amplitude of the wave. Similarly, in GID a photon diffracted from a modified surface of the sample can interfere with photons diffracted from the bulk of the sample, creating thickness oscillations which consequently modulate the specular reflectivity.

In Chapter 4 we analyze these modulations of specular reflectivity, and how they depend on the crystal structure of the sub-surface with respect to depth. We show that these modulations potentially can be used for the crystal surface characterization using lab-based instruments unlike standard implementations of GID using large-scale, but difficult-to-access synchrotron radiation sources.

\subsubsection{Artificial 3D shaped nanoscale systems}

A common feature of the above techniques is that they are directly sensitive to the spatial distribution of the electron density. The photon energy of XRF is characteristic to each element, hence XRF is directly sensitive to the probe beam-averaged elemental composition of the irradiated sample. That is the basis of XRF spectroscopy. There are techniques sensitive to the spatial distribution of the electron density and techniques that are sensitive to the spatially averaged elemental composition. We now focus on the analysis which is selectively sensitive to the spatial distributions of different elements.

Such analysis is possible using the X-ray standing wave (XSW) technique: transmitted and elastically scattered X-rays interfere, generating a standing wave. The $\mathrm{X}$-ray standing wave is represented by a time-independent spatial distribution of the amplitude inside the sample, with minima and maxima in the nodes and anti-nodes of the standing wave, respectively. As an analogy, one can think of the periodic nano-scale structure as of an optical cavity, used in lasers to create a standing wave. However, unlike a simple optical cavity which simply consists of two parallel mirrors, nano-scale devices have a complex structure and as such the configuration of the XSW requires more advanced calculations. A configuration of the spatial distribution of the X-ray standing wave is dependent on the incidence angle and the parameters of the structure. Consequently, the probability of emission of XRF photons is spatially modulated in XSW analysis. By detecting intensities of characteristic XRF lines of different elements with respect to different incidence angles, one can analyze the spatial distribution of their atomic density. That constitutes the basis of the XSW technique (see comprehensive rewiev in [40]). In the pioneering works of Batterman [41, 42] XSW was used to study the Borrmann effect [43, 44] (anomalous transmittance of a crystal near the Bragg conditions). Nowadays it is a powerful tool to study the atomic structure of Langmuir-Blodgett films [45, 46], epitaxial films, thin film multilayers [47] and multilayer mirrors[48]. 
We now have discussed that XSW allows to resolve atomic concentration distributions of selected elements in depth. This is 1D element selective characterization of the structure in which the lateral distribution is averaged. However, with advances in science and technology the demand in analysis, capable of the genuine 3D characterization of the atomic distribution, is growing. This challenge is obvious in the work of Dialameh et.al. [22]. There, the structure of 3D Cr nano-columns were studied using grazing incidence XRF (GIXRF). Two samples were prepared by means of e-beam lithography [49]: the first one had a stochastic arrangement of nano-columns on top of a substrate, the second had a regular periodic arrangement of the columns. To study the lateral structure, the $\mathrm{Cr}-\mathrm{K} \alpha$ line intensity was measured, not only for various incidence angles, but also under various azimuthal orientations of the sample. It is expected that the lateral structure of the stochastically arranged sample is averaged and that the GIXRF curves are independent on the azimuthal orientation of the sample. Therefore, in this case, in-depth XSW characterization is sufficient. In contrast, in the case of periodically arranged structures, the GIXRF signal is strongly dependent on the azimuthal orientation, demonstrating the sensitivity to the lateral distribution of the element atomic concentration. Thus, there is a need for a mathematical model capable of taking into account the lateral structure of standing X-ray waves.

In recent work of Soltwisch et.al. [23] this problem is approached by using finite element (FEM) simulations. There, the $2 \mathrm{D}$ structure of $\mathrm{Si}_{3} \mathrm{~N}_{4}$ lamellar gratings was studied. GIXRF was measured under various azimuthal orientation of a grating for the N-K $\alpha$ line. The distribution of the XSW amplitude was calculated by solving the Helmholtz equation [50] using FEM [51] on a 2D mesh (depth and one lateral axis). Simulations of GIXRF curves for various azimuthal orientation showed good agreement, with the possibility to analyze the lateral distribution of elements. FEM has a major advantage of high precision, but also a disadvantage of high computational cost. This disadvantage significantly limits the application of FEM for 3D XSW analysis: in particular, the computational effort increases with the computational domain which depends on the wavelength of the incident beam and characteristic size of the structure. For instance, the wavelength of the incident radiation which is sufficient to excite the $\mathrm{N}-\mathrm{K} \alpha$ line, is an order of magnitude larger than the one needed to excite the $\mathrm{Cr}-\mathrm{K} \alpha$ line, as in the previous nano-columns example. The characteristic size of the $\mathrm{Si}_{3} \mathrm{~N}_{4}$ lamellar grating studied was less by a factor of 10. XSW analysis demands more computational effort for simulation of XSW induced by harder X-ray radiation (smaller wavelength) and larger structures. Concerning the 3D arrangement of nano-columns, this renders FEM calculation practically impossible for GIXRF analysis of Cr nano-columns. That necessitates the development of a novel calculation scheme for the XSW field. This request was also expressed in [23].

In Chapter 5 we present a semi-analytical, mathematical model based on the dynamical diffraction theory in many beam approximation [52] (also known as rigorous coupled wave analysis [53]). The semi-analytical nature of that theory allowed us to derive equations for the calculation of GIXRF intensity in linear-algebraic form. These equations negate the problem of computational effort for XSW analysis of systems as complex as 3D periodic structures. A new mathematical model is 
tested on the same experimental data published in [23] showing qualitatively similar quality of fit as compared with FEM calculations in [23]. Calculations using new computational scheme are faster by three orders of magnitude. Next, we implement our approach to the 3D arranged nano-columns structure and the simulations shows excellent quantitative agreement with experimental data published in [22].

Further, in Chapter 6 we compare near field calculations using a dynamical diffraction theory directly with FEM calculations. We choose a lamellar grating irradiated by an EUV beam as a benchmark model since it can be used in EUV lithography and since EUV requires less computational effort in TEM than the X-ray regime. Unlike the equations in Chapter 5, where for simplicity scalar approximation is used to solve the Helmholz equation, in Chapter 6 we rederive equations for the dynamical diffraction theory in the vector form. This approach shows a better agreement with FEM, which we believe to be more precise since its computational scheme is based on fundamental principles.

In conclusion of the status of short-wavelength metrology and the major analytical challenges described above, this thesis captures several analytical approaches to extend the scope of current X-ray and EUV analysis. This notably concerns the broadening of the dimenstionality of studied nano-scale devices and structures, and methods to deliver an enhanced precission by combining different wavelength ranges. 


\section{References}

[1] W. H. Bragg and W. L. Bragg, The reflection of X-rays by crystals, Proceedings of the Royal Society A: Mathematical, Physical and Engineering Sciences 88, 428 (1913).

[2] H. M. Rietveld, A profile refinement method for nuclear and magnetic structures, Journal of Applied Crystallography 2, 65 (1969).

[3] Q. Liu, D. E. Bugaris, G. Xiao, M. Chmara, S. Ma, H.-C. zur Loye, M. D. Amiridis, and $\mathrm{F}$. Chen, $\mathrm{Sr}_{2} \mathrm{Fe}_{1.5} \mathrm{Mo}_{0.5} \mathrm{O}_{6-\delta}$ as a regenerative anode for solid oxide fuel cells, Journal of Power Sources 196, 9148 (2011).

[4] S. S. Iyengar, N. V. Phadnis, and R. Suryanarayanan, Quantitative analyses of complex pharmaceutical mixtures by the rietveld method, Powder Diffraction 16, 20-24 (2001).

[5] A. Martucci, L. Pasti, N. Marchetti, A. Cavazzini, F. Dondi, and A. Alberti, Adsorption of pharmaceuticals from aqueous solutions on synthetic zeolites, Microporous and Mesoporous Materials 148, 174 (2012).

[6] T. Torres, V. Sagredo, L. de Chalbaud, G. Attolini, and F. Bolzoni, Magnetic and structural characterization of the semiconductor $\mathrm{FeIn}_{2} \mathrm{Se}_{4}$, Physica B: Condensed Matter 384, 100 (2006).

[7] H. Kiessig, Untersuchungen zur totalreflexion von röntgenstrahlen (in german), Annalen der Physik 402, 715 (1931).

[8] L. G. Parratt, Surface studies of solids by total reflection of X-rays, Phys. Rev. 95, 359 (1954).

[9] S. Wirths, D. Buca, Z. Ikonic, P. Harrison, A. Tiedemann, B. Holländer, T. Stoica, G. Mussler, U. Breuer, J. Hartmann, D. Grützmacher, and S. Mantl, SiGeSn growth studies using reduced pressure chemical vapor deposition towards optoelectronic applications, Thin Solid Films 557, 183 (2014).

[10] D. L. Windt, S. Donguy, C. J. Hailey, J. Koglin, V. Honkimaki, E. Ziegler, F. E. Christensen, H. Chen, F. A. Harrison, and W. W. Craig, W/SiC X-ray multilayers optimized for use above 100 keV, Appl. Opt. 42, 2415 (2003).

[11] P. Pisecny, K. Husekova, K. Frohlich, L. Harmatha, J. Soltys, D. Machajdik, J. Espinos, M. Jergel, and J. Jakabovic, Growth of lanthanum oxide films for application as a gate dielectric in CMOS technology, Materials Science in Semiconductor Processing 7, 231 (2004).

[12] C. Dornes, Y. Acremann, M. Savoini, M. Kubli, M. J. Neugebauer, E. Abreu, L. Huber, G. Lantz, C. A. F. Vaz, H. Lemke, E. M. Bothschafter, M. Porer, V. Esposito, L. Rettig, M. Buzzi, A. Alberca, Y. W. Windsor, P. Beaud, U. Staub, D. Zhu, S. Song, J. M. Glownia, and S. L. Johnson, The ultrafast Einstein-de Haas effect, Nature 565, 209 (2019). 
[13] E. Beaurepaire, J.-C. Merle, A. Daunois, and J.-Y. Bigot, Ultrafast spin dynamics in ferromagnetic nickel, Phys. Rev. Lett. 76, 4250 (1996).

[14] J. P. Zhang, M. A. Khan, W. H. Sun, H. M. Wang, C. Q. Chen, Q. Fareed, E. Kuokstis, and J. W. Yang, Pulsed atomic-layer epitaxy of ultrahigh-quality $\mathrm{Al}_{x} G a_{1-x} N$ structures for deep ultraviolet emissions below $230 \mathrm{~nm}$, Applied Physics Letters 81, 4392 (2002).

[15] I. A. Makhotkin, E. Zoethout, R. van de Kruijs, S. N. Yakunin, E. Louis, A. M. Yakunin, V. Banine, S. Müllender, and F. Bijkerk, Short period La/B and LaN/B multilayer mirrors for $6.8 \mathrm{~nm}$ wavelength, Opt. Express 21, 29894 (2013).

[16] B. Krause, D. S. Kuznetsov, A. E. Yakshin, S. Ibrahimkutty, T. Baumbach, and F. Bijkerk, In situ and real-time monitoring of structure formation during non-reactive sputter deposition of lanthanum and reactive sputter deposition of lanthanum nitride, Journal of Applied Crystallography 51, 1013 (2018).

[17] E. Metwalli, V. Körstgens, K. Schlage, R. Meier, G. Kaune, A. Buffet, S. Couet, S. V. Roth, R. Röhlsberger, and P. Müller-Buschbaum, Cobalt nanoparticles growth on a block copolymer thin film: A time-resolved GISAXS study, Langmuir 29, 6331 (2013).

[18] A. Mezei, R. Pons, and M. C. Morán, The nanostructure of surfactant-DNA complexes with different arrangements, Colloids and Surfaces B: Biointerfaces 111, 663 (2013).

[19] P. Ngabonziza, R. Heimbuch, N. de Jong, R. A. Klaassen, M. P. Stehno, M. Snelder, A. Solmaz, S. V. Ramankutty, E. Frantzeskakis, E. van Heumen, G. Koster, M. S. Golden, H. J. W. Zandvliet, and A. Brinkman, In situ spectroscopy of intrinsic $\mathrm{Bi}_{2} \mathrm{Te}_{3}$ topological insulator thin films and impact of extrinsic defects, Phys. Rev. B 92, 035405 (2015).

[20] O. Perales-Perez, A. Parra-Palomino, R. Singhal, P. M. Voyles, Y. Zhu, W. Jia, and M. S. Tomar, Evidence of ferromagnetism in $Z_{n_{1-x}} M_{x} O(M=N i, C u)$ nanocrystals for spintronics, Nanotechnology 18, 315606 (2007).

[21] K. Nakayama, Y. Ishimaru, H. Wakana, S. Adachi, Y. Tarutani, and K. Tanabe, Fabrication of high-quality multilayer structure for HTS-SFQ circuits using surface treatments, IEEE Transactions on Applied Superconductivity 15, 157 (2005).

[22] M. Dialameh, F. Ferrarese Lupi, P. Hönicke, Y. Kayser, B. Beckhoff, T. Weimann, C. Fleischmann, W. Vandervorst, P. Dubček, B. Pivac, et al., Development and synchrotron-based characterization of Al and $\mathrm{Cr}$ nanostructures as potential calibration samples for $3 D$ analytical techniques, physica status solidi (a) 215, 1700866 (2018).

[23] V. Soltwisch, P. Hönicke, Y. Kayser, J. Eilbracht, J. Probst, F. Scholze, and B. Beckhoff, Element sensitive reconstruction of nanostructured surfaces with 
finite elements and grazing incidence soft X-ray fluorescence, Nanoscale 10, 6177 (2018).

[24] E. Louis, A. Yakshin, T. Tsarfati, and F. Bijkerk, Nanometer interface and materials control for multilayer EUV-optical applications, Progress in Surface Science 86, 255 (2011).

[25] A. N. Shiryaev, Probability (Springer New York, 2016).

[26] L. D. Landau and E. M. Lifshitz, Quantum mechanics: non-relativistic theory, Vol. 3 (Elsevier, 2013).

[27] J. Daillant and A. Gibaud, X-ray and neutron reflectivity: principles and applications, Vol. 770 (Springer, 2008).

[28] R. Lazzari, IsGISAXS: a program for grazing-incidence small-angle X-ray scattering analysis of supported islands, Journal of Applied Crystallography 35, 406 (2002).

[29] M. Buljan, N. Radić, S. Bernstorff, G. Dražić, I. Bogdanović-Radović, and V. Holy, Grazing-incidence small-angle X-ray scattering: application to the study of quantum dot lattices, Acta Crystallographica Section A: Foundations of Crystallography 68, 124 (2012).

[30] R. Maruyama, D. Yamazaki, K. Akutsu, T. Hanashima, N. Miyata, H. Aoki, M. Takeda, and K. Soyama, Development of high-polarization Fe/Ge neutron polarizing supermirror: Possibility of fine-tuning of scattering length density in ion beam sputtering, Nuclear Instruments and Methods in Physics Research Section A: Accelerators, Spectrometers, Detectors and Associated Equipment 888, 70 (2018).

[31] A. P. Payne and B. M. Clemens, Influence of roughness distributions and correlations on X-ray diffraction from superlattices, Phys. Rev. B 47, 2289 (1993).

[32] V. Holỳ and T. Baumbach, Nonspecular X-ray reflection from rough multilayers, Physical Review B 49, 10668 (1994).

[33] V. Kaganer, S. Stepanov, and R. Köhler, Bragg diffraction peaks in X-ray diffuse scattering from multilayers with rough interfaces, Physical Review B 52, 16369 (1995).

[34] V. Kaganer, S. Stepanov, and R. Köhler, Effect of roughness correlations in multilayers on bragg peaks in X-ray diffuse scattering, Physica B: Condensed Matter 221, 34 (1996).

[35] W. C. Marra, P. Eisenberger, and A. Y. Cho, X-ray total-externalreflection-bragg diffraction: A structural study of the gaas-al interface, Journal of Applied Physics 50, 6927 (1979).

[36] P. L. Cowan, Diffraction of evanescent X-rays: Results from a dynamical theory, Phys. Rev. B 32, 5437 (1985). 
[37] V. A. Bushuev, R. M. Imamov, É. K. Mukhamedzhanov, and A. P. Oreshko, Specular reflection of X-rays under the conditions of grazing diffraction in a crystal with an amorphous surface layer, Crystallography Reports 46, 909 (2001).

[38] V. A. Bushuev and A. P. Oreshko, Specular X-ray reflection from a crystal coated with an amorphous film under the conditions for strongly asymmetric noncoplanar diffraction, Physics of the Solid State 43, 941 (2001).

[39] V. A. Bushuev and A. P. Oreshko, X-ray specular reflection under conditions of extremely asymmetric noncoplanar diffraction from a bicrystal, Crystallography Reports 48, 180 (2003).

[40] Z. Jörg and A. Kazimirov, X-ray Standing Wave Technique, The: Principles and Applications (Series on Synchrotron Radiation Techniques and Applications) (World Scientific Pub Co Inc, 2013).

[41] B. W. Batterman, Effect of dynamical diffraction in X-ray fluorescence scattering, Phys. Rev. 133, A759 (1964).

[42] B. W. Batterman and H. Cole, Dynamical diffraction of $x$ rays by perfect crystals, Rev. Mod. Phys. 36, 681 (1964).

[43] G. Borrmann, Die absorption von röntgenstrahlen im fall der interferenz (in german), Zeitschrift für Physik 127, 297 (1950).

[44] E. J. Saccocio and A. Zajac, Simultaneous diffraction of $x$ rays and the borrmann effect, Phys. Rev. 139, A255 (1965).

[45] M. Bedzyk, D. Bilderback, G. Bommarito, M. Caffrey, and J. Schildkraut, $X$-ray standing waves: a molecular yardstick for biological membranes, Science 241, 1788 (1988).

[46] N. N. Novikova, S. I. Zheludeva, O. V. Konovalov, M. V. Kovalchuk, N. D. Stepina, I. V. Myagkov, Y. K. Godovsky, N. N. Makarova, E. Y. Tereschenko, and L. G. Yanusova, Total reflection X-ray fluorescence study of langmuir monolayers on water surface, Journal of Applied Crystallography 36, 727 (2003).

[47] I. Kröger, B. Stadtmüller, C. Kleimann, P. Rajput, and C. Kumpf, Normalincidence $X$-ray standing-wave study of copper phthalocyanine submonolayers on cu(111) and au(111), Phys. Rev. B 83, 195414 (2011).

[48] S. N. Yakunin, I. A. Makhotkin, R. W. E. van de Kruijs, M. A. Chuev, E. M. Pashaev, E. Zoethout, E. Louis, S. Y. Seregin, I. A. Subbotin, D. V. Novikov, F. Bijkerk, and M. V. Kovalchuk, Model independent X-ray standing wave analysis of periodic multilayer structures, Journal of Applied Physics 115, 134303 (2014).

[49] M. Altissimo, E-beam lithography for micro-/nanofabrication, Biomicrofluidics 4, 026503 (2010). 
[50] L. D. Landau, L. P. Pitaevskii, and E. Lifshitz, Electrodynamics of Continuous Media: Volume 8 (Course of Theoretical Physics) (Butterworth-Heinemann, 1984).

[51] J. Pomplun, S. Burger, L. Zschiedrich, and F. Schmidt, Adaptive finite element method for simulation of optical nano structures, physica status solidi (b) 244, 3419 (2007).

[52] P. Mikulík and T. Baumbach, X-ray reflection by rough multilayer gratings: Dynamical and kinematical scattering, Phys. Rev. B 59, 7632 (1999).

[53] J. J. Hench and Z. Strakoš, The RCWA method - a case study with open questions and perspectives of algebraic computations, Vol. 31 (Institute of Computational Mathematics, 2008) pp. 331-357. 



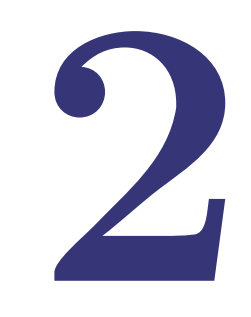

\section{COMBINED EUV AND X-RAY}

\section{ANALYSIS OF PERIODIC MULTILAYER STRUCTURES}

We present a way to analyze the chemical composition of periodical multilayer structures using the simultaneous analysis of grazing incidence hard X-Ray reflectivity $(G I X R)$ and normal incidence extreme ultraviolet reflectance (EUVR). This allows to combine the high sensitivity of GIXR data to layer and interface thicknesses with the sensitivity of EUVR to the layer densities and atomic compositions. This method was applied to the reconstruction of the layered structure of a LaN/B multilayer mirror with $3.5 \mathrm{~nm}$ periodicity. We have compared profiles obtained by simultaneous EUVR and GIXR and GIXR-only data analysis, both reconstructed profiles result in a similar description of the layered structure. However, the simultaneous analysis of both EUVR and GIXR by a single algorithm lead to $a \sim 2 x$ increased accuracy of the reconstructed layered model, or a more narrow range of solutions, as compared to the GIXR analysis only. It also explains the inherent difficulty of accurately predicting EUV reflectivity from a GIXR-only analysis. 


\subsection{Introduction}

Layered materials find many applications nowadays. These range from nanomaterials in general to XUV reflecting Bragg optics, also down to atomic scale dimensions. Traditional characterization of such, periodic multilayer mirrors usually involves two types of measurements of the reflectance, one performed using hard X-rays at grazing incidence (GIXR), and a second performed at an application relevant wavelength. A structural model obtained from hard X-ray reflectometry analysis is generally not able to accurately predict the application relevant reflectivity data. The reason for this is that the reflectivities at different wavelengths have different sensitivities to the multilayer structural parameters. For example, while hard Xrays are very sensitive to the layer thicknesses in the multilayer period, it is less sensitive to the chemical composition of the layers. Soft X-rays are extremely sensitive to the compositional parameters of the layers, such as stoichiometry and the presence of impurity atoms, but the analysis of such data suffers from the large correlation between model parameters that describe the measurement curves.

A recent attempt to obtain a consistent model of a multilayer period structure that describes both hard X-ray reflectivity (GIXR, $0.154 \mathrm{~nm}$ ) and extreme ultraviolet reflectivity (EUVR, $\sim 6.7 \mathrm{~nm}$ ) measurements is discussed in [1]. In that article authors have analyzed sequentially GIXR and EUVR data, concluding that interface roughness values for the model that describes EUVR should be higher than interface roughness values for the model that describes GIXR data. One of the possible reasons of inconstancy between these models was attributed to neglecting the atomic composition of diffused layers.

In this chapter we will discuss simultaneous fitting of GIXR and EUVR data using a single model that simultaneously describes both sets of data. This approach is expected to result in a reliable and accurate model of the multilayer structure that provides more accurate information about the internal structure, as well as enabling a more accurate prediction of the reflectivity of multilayers with changing model parameters such as a variation of the multilayer period thickness or a variation of the number of periods as discussed in [2, 3]. In order to account for the different sensitivities of X-rays and EUV radiation to the chemical composition of the layers, we propose to add the chemical composition of layers and interfaces as a parameters during the combined fit of GIXR and EUVR data.

Basic mathematical techniques optimized for the simulation of reflectivity data for a periodic multilayer structure are discussed. To study the benefits of using several sets of data for the reconstruction of the material parameters such as densities and atomic compositions of layers, we have performed an extensive analysis of errors of the reconstructed optical constants profiles and correlations between fit parameters. To illustrate the performance of a combined GIXR and EUVR analysis, we will analyze a LaN/B multilayer optimized for normal incidence reflectivity at a wavelength of $6.8 \mathrm{~nm}$ and discuss reconstructed profiles from GIXR-only and from combined GIXR and EUVR analysis. This material combination is of particular interest because of its current application as spectroscopic element in XRF analysis equipment and its potential application as reflective optical element in next generation EUV photolithography. 


\subsection{Modeling of reflectivity from periodic multilayer structures}

In this part we present a brief description of electromagnetic wave propagation, optimized for fast calculation of the reflectivity from a periodic multilayer structure. The wave propagation in a homogeneous layer can be characterized using the transfer matrix [4] $M_{i}$ that connects the electric field and it's first derivative at the interfaces between neighboring layers $i$ and $i+1$ :

$$
\mathrm{M}_{i}=\left(\begin{array}{cc}
\cos k_{z, i} d_{i} & 1 / k_{z, i} \sin k_{z, i} d_{i} \\
-k_{z, i} \sin k_{z, i} d_{i} & \cos k_{z, i} d_{i}
\end{array}\right),
$$

where $d_{i}$ is the layer thickness and $k_{z, i}$ is a projection of the wave vector on to the $z$-direction in layer $i$. In general case, $k_{z, i}$ depends on the polarization [5] of the incident radiation:

$$
k_{z, i}= \begin{cases}k_{0} \sqrt{n_{i}^{2}-n_{0}^{2} \cos ^{2} \theta} & \text { s polarization; } \\ k_{0} n_{i}^{2} / \sqrt{n_{i}^{2}-n_{0}^{2} \cos ^{2} \theta} & \text { p polarization }\end{cases}
$$

where $n_{i}=1-\delta_{i}-i \beta_{i}$ is the complex refractive index inside layer $i[6], k_{0}=\left|\mathbf{k}_{0}\right|=$ $2 \pi / \lambda$ is the absolute value of the wave vector in vacuum, $\lambda$ is the incident beam wavelength and $\theta$ is the grazing incident angle.

The wave propagation through a system with $N$ layers is then represented by the characteristic matrix:

$$
\mathrm{M}=\mathrm{M}_{N} \mathrm{M}_{N-1} \cdots \mathrm{M}_{2} \mathrm{M}_{1}=\prod_{i=N}^{1} \mathrm{M}_{i}
$$

For periodic multilayer structures with identical periods, the multiplication of matrices $M_{i}$ can be calculated via the exponentiation formula [4]:

$$
\begin{aligned}
\tilde{\mathrm{M}}^{K}=\left(\begin{array}{ll}
\tilde{m}_{11} & \tilde{m}_{12} \\
\tilde{m}_{21} & \tilde{m}_{22}
\end{array}\right)^{K}= \\
\quad=\left(\begin{array}{cc}
\tilde{m}_{11} U_{K-1}(a)-U_{K-2}(a) & \tilde{m}_{12} U_{K-1}(a) \\
\tilde{m}_{21} U_{K-1}(a) & \tilde{m}_{22} U_{K-1}(a)-U_{K-2}(a)
\end{array}\right) .
\end{aligned}
$$

Here $U_{K}(a)=\sin [(n+1) \arccos a] / \sqrt{1-a^{2}}$ is the Chebyshev polynomial of the second kind [7], where $a=1 / 2\left(\tilde{m}_{11}+\tilde{m}_{22}\right), \widetilde{M}$ is the characteristic matrix calculated for a multEilayer period and $K$ is the number of periods in a multilayer structure. This approach is valid for unimodular matrices and can be applied to the characteristic matrices discussed here because $\operatorname{det}\left(\mathrm{M}_{i}\right)=1$. Using Chebyshev polynomials allows to save computational resources, proportionally to the number of periods in a multilayer stack in comparison with standard matrix multiplication procedures in Eq. 2.3.

The reflectance amplitude is now given by [4]:

$$
r=\frac{k_{z, N+1} k_{z, 0} M_{12}+i k_{z, 0} M_{22}-i k_{z, N+1} M_{11}+M_{21}}{k_{z, N+1} k_{z, 0} M_{12}+i k_{z, 0} M_{22}+i k_{z, N+1} M_{11}-M_{21}},
$$


where $k_{z, 0}$ and $k_{z, N+1}$ are the wave vector projections in ambient and substrate media respectively. Reflected beam intensity can then be calculated by:

$$
I^{\text {calc }}(\theta, \lambda, \mathbf{p})=|r|^{2} I_{0},
$$

where $\mathbf{p}$ is the set of structural parameters (layer thicknesses and refractive indices) and $I_{0}$ is the incident beam intensity. Formulas Eq. 2.1 - Eq. 2.6 will further be used for model simulations of GIXR and EUVR curves.

\subsection{Parameterization of a multilayer structure}

For simulations analysis of GIXR and EUVR data, Eq. 2.6 can be written as

$$
I=\left\{\begin{array}{l}
\left.I_{\mathrm{GIXR}}^{\mathrm{calc}}(\theta, \lambda, \mathbf{p})\right|_{\lambda=\lambda_{0}} ; \\
\left.I_{\mathrm{EUVR}}^{\text {calc }}(\theta, \lambda, \mathbf{p})\right|_{\theta=\theta_{0}},
\end{array}\right.
$$

where $\lambda_{0}$ is a fixed wavelength used for the measurements of GIXR, and $\theta_{0}$ is a fixed angle used for measurements of EUVR. According to Eq. 2.1 - Eq. 2.6, a multilayer is described by a set of individual layers with thicknesses $d_{i}$ and complex refractive indices $n_{i}$.

The refractive index of the $i$-th layer $\left(n_{i}=1-\delta_{i}-i \beta_{i}\right)$ depends on it's chemical composition and density according to [8]:

$$
\begin{aligned}
& \delta_{i}=2.7007 \times 10^{-4} \times \frac{\rho_{i} \lambda^{2}}{\mu_{i}} \sum_{j=1}^{\Omega_{i}} \omega_{i j} f_{j}^{(1)}(\lambda) ; \\
& \beta_{i}=2.7007 \times 10^{-4} \times \frac{\rho_{i} \lambda^{2}}{\mu_{i}} \sum_{j=1}^{\Omega_{i}} \omega_{i j} f_{j}^{(2)}(\lambda) .
\end{aligned}
$$

Here $\rho_{i}$ is the density, $\mu_{i}$ is the molar weight of a compound with $\Omega_{i}$ different atomic species, $\omega_{i j}$ is the atomic concentration of atoms $j$ in layer $i$, and $f_{j}$ is the atomic scattering factor for atomic species $j[9]$.

Although $\mathbf{p}$ contains the thicknesses needed to describe the layered model for reflectivity simulations, periodic multilayer mirrors are often described using technological parameters such as period thickness $D$ and layer thickness ratio $\Gamma$ instead of the individual layer thicknesses $d_{i}$. In analogy to the technological parameters $D$ and $\Gamma$ we introduce the relative (to the period) interface imperfections parameter $S$ and the interface imperfections ratio parameter $S_{\Gamma}$. Thus for a two-layer model, it is convenient to use a set of effective parameters:

$$
\left\{\begin{array}{l}
D=d_{1}+d_{2}+\sigma_{1}+\sigma_{2} \\
\Gamma=\left(2 d_{2}+\sigma_{1}+\sigma_{2}\right) / 2 D \\
S=\left(\sigma_{1}+\sigma_{2}\right) / D \\
S_{\Gamma}=\sigma_{2} /\left(\sigma_{1}+\sigma_{2}\right)
\end{array}\right.
$$

Interface imperfections between layers $i$ and $i+1$, resulting from intermixing and/or surface roughness over a depth range of $\sigma_{i}$, effectively create a gradual change in 
$\delta$ and $\beta$ from layer $i$ to layer $i+1$. This gradual change is taken into account in the model by replacing this depth range $\sigma_{i}$ by a finite set of layers with total thickness $\sigma_{i}$ that introduce a gradual stepwise profile from $\delta_{i}$ to $\delta_{i+1}$ and from $\beta_{i}$ to $\beta_{i+1}$ [10]. Here the profile is chosen according to a sinusoidal distribution of optical characteristics between homogeneous media. This approach maintains the continuity of the electric field at the interfaces and properly considers dynamic effects, unlike the commonly used Debye-Waller or Nevot-Croce statistical factors [5, 11]. Also it takes into account the shift of the diffraction peaks caused by interface imperfections. Furthermore this description of the interfaces does not affect the unimodularity condition for the characteristic matrix Eq. 2.1, and therefore allows the application of the exponentiation formula Eq. 2.4.

\subsection{Reconstruction and error analysis of structural parameters}

The reconstruction of the structural parameters is formulated as an optimization problem [12]:

$$
\tilde{\mathbf{p}}=\min _{\mathbf{p}} \chi^{2}(\mathbf{p})
$$

where $\tilde{\mathbf{p}}$ is a resulting set of reconstructed parameters and $\chi^{2}$ is a goodness of fit value similar to Pearson's criterion.

In order to reconstruct parameters from two sets of experimental data the criterion for fit goodness has the form:

$$
\begin{aligned}
\chi^{2}=\frac{1}{L_{\mathrm{GIXR}}+L_{\mathrm{EUVR}}-l}\left[\sum_{\theta} \frac{\left(\left.I_{\mathrm{GIXR}}^{\mathrm{calc}}(\theta, \mathbf{p})\right|_{\lambda=\lambda_{0}}-I_{\mathrm{GIXR}}^{\exp }(\theta)\right)^{2}}{\sigma_{\mathrm{GIXR}}^{2}(\theta)}+\right. \\
\left.+\sum_{\lambda} \frac{\left(\left.I_{\mathrm{EUVR}}^{\mathrm{calc}}(\lambda, \mathbf{p})\right|_{\theta=\theta_{0}}-I_{\mathrm{EUVR}}^{\exp }(\lambda)\right)^{2}}{\sigma_{\mathrm{EUVR}}^{2}(\lambda)}\right],
\end{aligned}
$$

where $L_{\mathrm{GIXR}}$ and $L_{\mathrm{EUVR}}$ are numbers of measured data points, $l$ is the number of parameters that are used to describe the layered structure, and $\sigma_{\text {GIXR }}$ and $\sigma_{\text {EUVR }}$ are the uncertainties in the measured GIXR and EUVR data respectively. Both $\sigma_{\text {GIXR }}$ and $\sigma_{\text {EUVR }}$ are calculated according to $\sigma^{2}(\theta)=\sigma_{\text {sys }}^{2}+\sigma_{\text {stat }}^{2}$, where $\sigma_{\text {sys }}$ is a systematic error that relates to uncertainties in the measurement setup, and $\sigma_{\text {stat }}$ is the statistical error in the measured data relates to the discrete nature of radiation. If errors in the experimental data are normally distributed and the number of experimental points is much larger than the number of fit parameters, a goodness of fit for a perfect model has a value of $\chi^{2}=1$.

In order to solve the optimization problem of Eq. 2.10, a Levenberg-Marquardt algorithm is used [12]. Standard deviations of reconstructed parameters $\Delta p_{i}$ are calculated by the least squares method [13]. To estimate standard deviations the covariance matrix $\mathbf{C}$ is used [14] which can be calculated as inverse modifyed Hessian 
matrix $\mathbf{C}=\mathbf{H}^{-1}$, where elements of modifyed Hessian have a form:

$$
H_{i j}=\sum_{k=1}^{L} \frac{1}{\sigma_{k}^{2}} \frac{\partial I_{k}}{\partial p_{i}} \frac{\partial I_{k}}{\partial p_{j}} .
$$

In case of simultaneous analysis of GIXR and EUVR data the concatenation of two experimental data sets is taken into account in Eq. 2.12. Consequently $I=$ $\left(I_{\mathrm{GIXR}}, I_{\mathrm{EUVR}}\right)$ is a cumulative set of measured data, $\sigma=\left(\sigma_{\mathrm{GIXR}}, \sigma_{\mathrm{EUVR}}\right)$ is a cumulative error, and $L=L_{\mathrm{GIXR}}+L_{\mathrm{EUVR}}$ is the total number of data points. Structural parameters $p_{i}$ are considered as normally distributed random variables to obtain standard deviations:

$$
\Delta p_{i}=\sqrt{C_{i i}}
$$

The degree of linear dependency of the parameters is determined by the matrix of Pearson correlation coefficients [12]:

$$
R_{i j}=\frac{C_{i j}}{\sqrt{C_{i i} C_{j j}}} .
$$

Elements of matrix $R$ are ranging from -1 to 1 . As an example a large absolute value of correlation coefficient $\left|R_{i j}\right|$ implies a large dependence between structural parameters $p_{i}$ and $p_{j}$. If $R_{i j}>0$, an increase of parameter $p_{i}$ can be compensated by an increase of parameter $p_{j}$ and vice versa, keeping the same $\chi^{2}$ value. If $R_{i j}<0$, an increase of parameter $p_{i}$ can be compensated by a decrease of the parameter $p_{j}$ and vice versa.

Based on the reconstructed parameters of the structure, one can obtain the depth distribution of the dispersion parameter $\delta(z)$. For the analysis of $\delta(z)$ the uncertainties correlation analysis is used [15]:

$$
\varepsilon(z)=\left[\sum_{i, j} \frac{\partial \delta(z)}{\partial p_{i}} \frac{\partial \delta(z)}{\partial p_{j}} C_{i j}\right]^{1 / 2}
$$

\subsection{Experiment layout and data processing}

A simultaneous analysis of GIXR and EUVR was performed for a 50 period LaN/B multilayer. Both La and B were deposited using DC magnetron sputtering. The LaN layer was created using nitrogen assisted growth similar to the approach described in [16]. The layer thicknesses were controlled by pre-calibrated deposition rates.

For the detailed analysis of the accuracy of the measurements it is essential to take all uncertainties into account. Unlike statistical error which strictly depends on experimental data, a systematic error is included that arises from the specific geometry of experimental setup.

The hard X-ray reflectivity measurements were carried out on a laboratory difractometer (PanAlytical Empyrean) using the characteristic $\mathrm{CuK}_{\alpha 1}$ radiation with a wavelength of $\lambda=0.15406 \mathrm{~nm}$. The monochromatization and primary collimation of the incident beam was done using a four bounce asymmetricaly cut 
germanium monochromator which gives a beam divergence of $\Delta \theta \approx 0.015^{\circ}$. For the calculation of errors we have also taken into account the fluctuation of the direct beam within $-2.5 \%$ of intensity, and possible errors in determination of incidence angle of $\Delta \theta \approx 0.017^{\circ}$.

The geometry of the experimental scheme, the cross section of the beam and the sample size were used for calculation of geometrical effects near the angle of total external reflection for GIXR data analysis. The measurement of EUV reflectivity was performed at PTB (Physikalisch-Technische Bundesanstalt) [17-19]. The accuracy of measurement was: intensity stability $-0.02 \%$; fluctuations in the detector $-0.04 \%$; the presence of high-order harmonics $-0.02 \%$; diffusely scattered radiation $-0.08 \%$. The total systematic error did not exceed $0.1 \%$.

To reconstruct the multilayer structure, the calculations of GIXR and EUVR data were fitted to the measured data. Initially only GIXR data were fitted, having effective parameters Eq. 2.9 and layer densities as free parameters. The fit model consisted of 49 periods with identical parameters and one additional top period with independent parameters to account for the effect of surface contamination (e.g. oxidation). The best fit model from GIXR analysis was subsequently used as the initial model for the simultaneous fit of GIXR and EUVR data, where the material compositions of layers are added as additional fit parameters.

For the analysis of a LaN/B multilayer, the LaN layer composition is defined as $(\mathrm{LaN})_{\omega_{\mathrm{LaN}}} \mathrm{B}_{1-\omega_{\mathrm{LaN}}}$ and the $\mathrm{B}$ layer composition is defined as $\mathrm{B}_{\omega_{\mathrm{B}}}(\mathrm{LaN})_{1-\omega_{\mathrm{B}}}$. Especially for a wavelength in the vicinity of the B-K $\mathrm{K}_{\alpha}$ absorption edge the EUVR simulations are very sensitive to the B optical constants [20, 21] and therefore to the B layer composition. For calculations of EUVR measured boron optical constants were used [21].

To estimate uncertainties in reconstructed parameters, standard deviations of fit parameters were calculated according to Eq. 2.13. Matrices of Pearson correlation coefficients are calculated for GIXR, EUVR and cumulative fits using Eq. 2.14, in order to analyze the stability of the solution of the optimization problem. The correlation matrix is calculated separately for each experiment to analyze sensitivity of the various experimental techniques to the parameters of the structure.

\subsection{Results}

The results of GIXR-only fitting are shown in Fig. 2.1 and the parameters of best fit models are presented in Table 2.1. Fig. 2.1 shows experimental data and best fit calculations, as well as the residuals $u=\left(I_{\exp }-I_{\text {calc }}\right) / \sigma$. The good agreement between fit calculations and experimental data can be recognized from the residuals that stay well within a range of $(-3 \div 3)$, and the fit quality value of $\chi^{2}=1.01$.

Fig. 2.2 shows measured and calculated EUVR curves. The dashed reflectivity curve was calculated based on the model obtained after the GIXR-only fit. Although the GIXR curve was fitted almost perfectly, the calculated EUVR curve does not fit to the measurements at all. It is clear that the structure parameters obtained from a GIXR-only fit are not sufficient to predict the multilayer characteristics in the EUV range. 


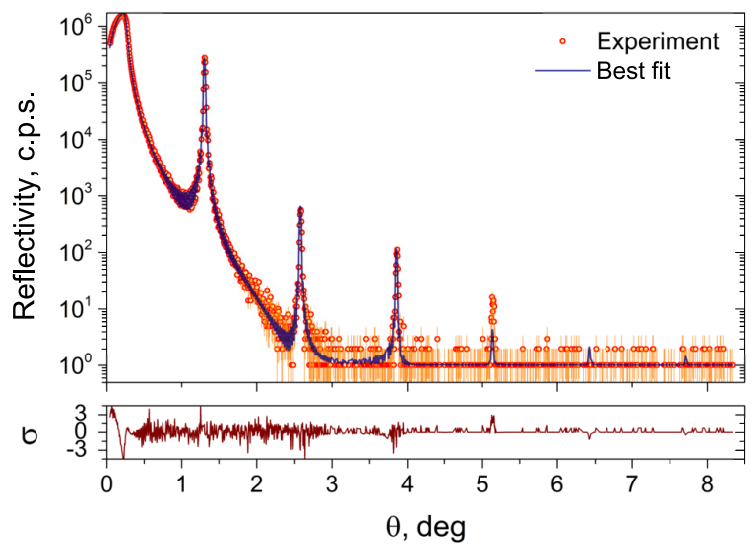

Figure 2.1: Calculated and measured GIXR curves for a LaN/B multilayer (top section), and the fit residuals (bottom section).

When a simultaneous analysis of GIXR and EUVR data is performed, the EUVR data can be reproduced accurately, as shown in Fig. 2.2 (solid line). The fit quality of the GIXR data, as obtained from the simultaneous GIXR and EUVR analysis, remained similar to that shown in Fig. 2.1. Resulting fit parameters from the simultaneous analysis are also shown in Table 2.1.

To explore the discrepancy between the calculated EUVR response from GIXRonly and simultaneous GIXR and EUVR analysis, the $\delta$-profiles and their tolerance areas were calculated, based on the parameters presented in Table 2.1. The tolerance areas are calculated using Eq. 2.15. The $\delta$-profiles and their tolerance areas were calculated for two wavelengths: $0.15 \mathrm{~nm}$ and $6.8 \mathrm{~nm}$. The $\delta$-profiles calculated for a wavelength of $0.15 \mathrm{~nm}$ are indicated as $\delta_{\mathrm{CuK}}$ in Fig. 2.3a. Profiles that are calculated

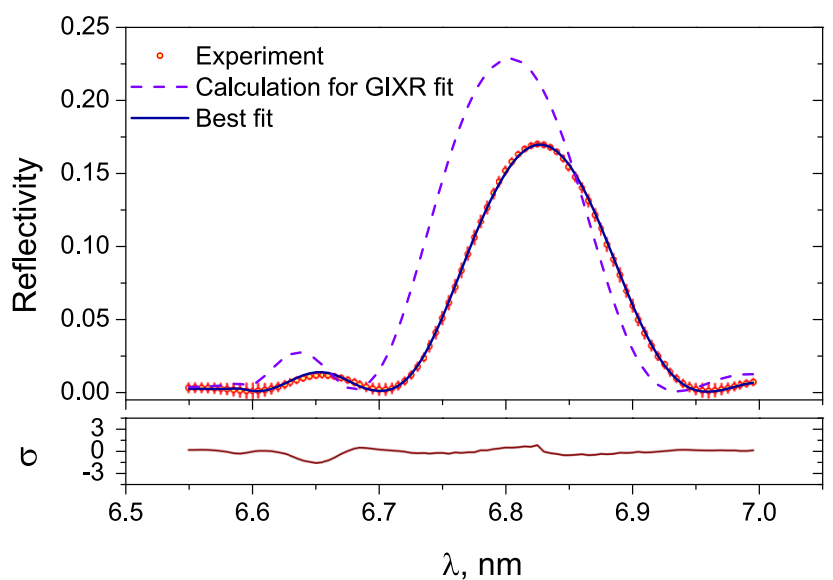

Figure 2.2: Calculated (see text for details) and measured curves for EUVR fit (top section) and the residual between best fit solution and the measured data (bottom section). 

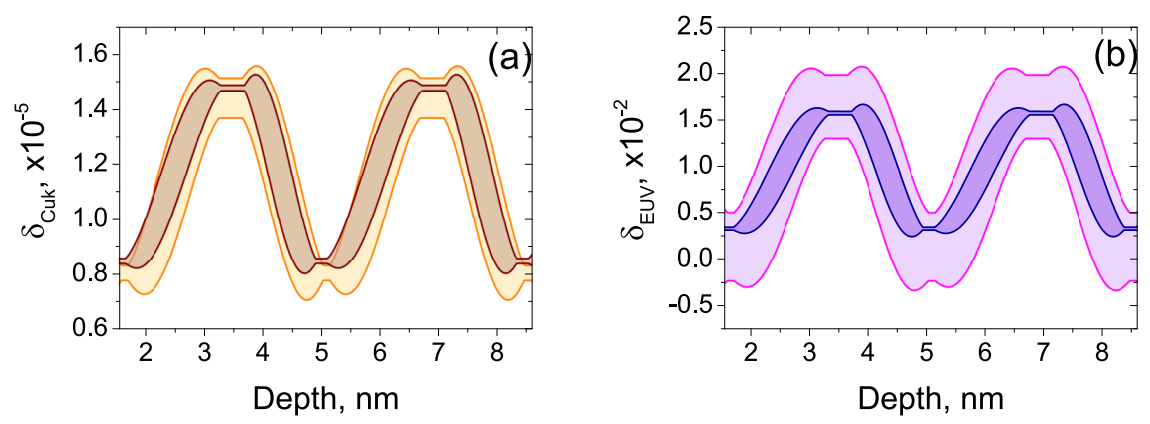

Figure 2.3: Tolerance areas of $\delta$-profiles of double period, calculated for $0.154 \mathrm{~nm}$ (a) and $6.8 \mathrm{~nm}$ (b) obtained for GIXR data fit (light) and cumulative GIXR and EUVR fit (dark).

Table 2.1: Resulting model of the periodic multilayer structure:

\begin{tabular}{c|c|c}
\hline & GIXR & Simultaneous \\
\hline \hline$D, \mathrm{~nm}$ & $3.432 \pm 0.001$ & $3.434 \pm 0.001$ \\
$\Gamma$ & $0.542 \pm 0.005$ & $0.529 \pm 0.004$ \\
$S$ & $0.85 \pm 0.01$ & $0.846 \pm 0.006$ \\
$S_{\Gamma}$ & $0.529 \pm 0.007$ & $0.573 \pm 0.005$ \\
$\rho_{\mathrm{B}}, \mathrm{g} / \mathrm{cm}^{3}$ & $2.7 \pm 0.2$ & $2.94 \pm 0.07$ \\
$\rho_{\mathrm{LaN}}, \mathrm{g} / \mathrm{cm}^{3}$ & $5.4 \pm 0.6$ & $5.58 \pm 0.14$ \\
$\omega_{\mathrm{B}}$ & $1.00 \pm 0.04$ & $0.977 \pm 0.002$ \\
$\omega_{\mathrm{LaN}}$ & $1.0 \pm 0.7$ & $1.00 \pm 0.03$ \\
\hline
\end{tabular}

for a wavelength of $6.8 \mathrm{~nm}$ are indicated as $\delta_{\mathrm{EUV}}$ in a Fig. 2.3b. The profiles that were calculated for a structural model obtained from the GIXR-only analysis will be referred to further as $\delta_{\mathrm{CuK}}^{\mathrm{G}}$ and $\delta_{\mathrm{EUV}}^{\mathrm{G}}$, while the profiles that correspond to the simultaneous GIXR and EUVR analysis will be referred to as $\delta_{\mathrm{CuK}}^{\mathrm{S}}$ and $\delta_{\mathrm{EUV}}^{\mathrm{S}}$.

The profiles can be divided into two types of regions, one region where the value of $\delta$ is constant, related to the thicknesses $d_{1}$ and $d_{2}$ of the LaN and B layers respectively, and another region where a gradual transition of $\delta$ occurs between the LaN and B layers and between the B and LaN layers, corresponding to the interface widths $\sigma_{1}$ and $\sigma_{2}$, respectively.

\subsection{Discussion}

The comparison of $\delta_{\mathrm{CuK}}^{\mathrm{G}}$ and $\delta_{\mathrm{CuK}}^{\mathrm{S}}$ profiles as plotted in Fig. 2.3a explains why the fit quality of GIXR was not changed. The solution of the simultaneous fit stays within the tolerance corridor of the solution of the GIXR-only fit. In Fig. 2.3a we can also see that the introduction of EUVR data into the analysis strongly increases the accuracy of the determination of optical constants, in particular at the position of the La and B layers. Table 2.1 shows that after the simultaneous fit, the error in 

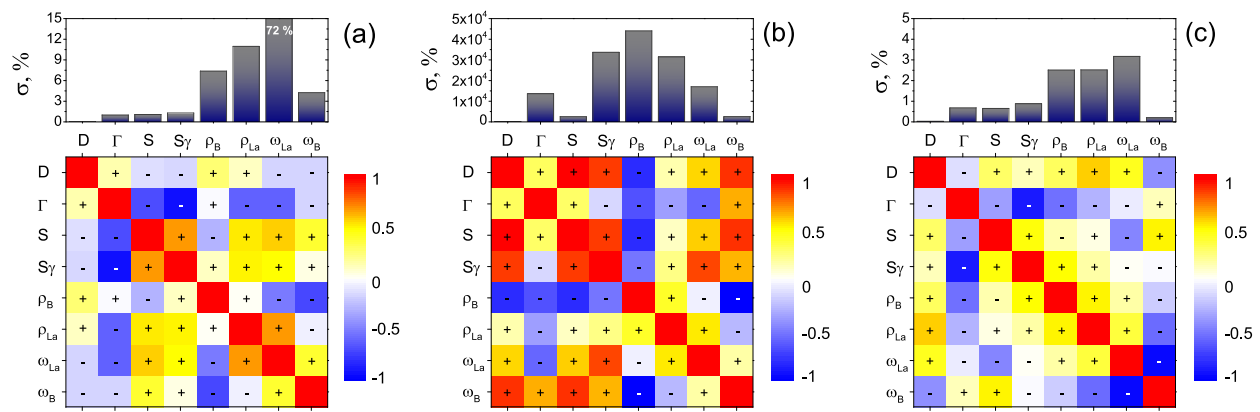

Figure 2.4: (top section) The relative errors of structural parameters. (bottom section) Matrices of Pearson's correlation coefficients. (left) for GIXR, (middle) for EUVR and (right) for simultaneous optimization.

the determination of densities decreases significantly.

The comparison of $\delta_{\mathrm{EUV}}^{\mathrm{G}}$ and $\delta_{\mathrm{EUV}}^{\mathrm{S}}$ profiles shows that within the tolerance corridor of the $\delta_{\mathrm{EUV}}^{\mathrm{G}}$, a large variety of optical profiles calculated for $6.8 \mathrm{~nm}$ wavelength can be placed. The tolerance corridor of $\delta_{\mathrm{EUV}}^{\mathrm{S}}$ is dramatically narrower than that of $\delta_{\mathrm{EUV}}^{\mathrm{G}}$.

The $\delta_{\mathrm{EUV}}^{\mathrm{G}}$ profile, which corresponds to the best fit model of GIXR-only analysis, does not fit into the $\delta_{\mathrm{EUV}}^{\mathrm{S}}$ corridor, which explains the poor prediction of EUVR data from the GIXR-only analysis as shown in Fig. 2.2. The main reason for the large tolerance regions of $\delta_{\mathrm{EUV}}^{\mathrm{G}}$ is that a variation of $\omega_{\mathrm{LaN}}, \omega_{\mathrm{B}}, \rho_{\mathrm{LaN}}$ and $\rho_{\mathrm{B}}$ parameters would lead to only a small change in $\delta_{\mathrm{CuK}}$ while leading to much larger changes in $\delta_{\mathrm{EUV}}$.

Fig. 2.4 shows the errors of the determined parameters as well as Pearson's correlation coefficient matrices, calculated using Eq. 2.14, for the GIXR (a), EUVR (b) and simultaneous (c) GIXR and EUVR analysis. Although we did not fit EUVR curves separately, we have calculated errors of possible EUVR-only fit for discussions. From Fig. 2.4a it can be concluded that the effective parameters $(D, \Gamma, S$ and $S_{\Gamma}$ ) are determined with high accuracy from the GIXR-only fit. Specifically the period $D$ of the multilayer mirror can be determined within an uncertainty of $\varepsilon D \approx 0.01 \%$. This high accuracy can be explained due to the fact that $D$ is strongly associated with the angular positions of the diffraction peaks, where a slight change in $D$ leads to a large change in $\chi^{2}$. As shown in Fig. 2.4a the parameter $D$ is only weakly correlated with other parameters. This is due the fact that shifting peaks position cannot be compensated by the change of other structural parameters.

Effective parameters $\Gamma, S$ and $S_{\Gamma}$ determine the shape of the $\delta_{\mathrm{CuK}}$-profile which determines the intensity ratio of the diffraction peaks. The accuracy with which these parameters can be determined from the GIXR analysis alone is typically in the order of $\varepsilon \approx 0.1 \%$. One can notice that the correlation between $\Gamma, S$ and $S_{\Gamma}$ is much larger than between $D$ and the other parameters. This is related to the fact that a change in the layer asymmetry parameter $\Gamma$ can be partially compensated by a change in the interface parameters $S$ and $S_{\Gamma}$. This large correlation explains the large tolerance areas in the interface regions of $\delta_{\mathrm{CuK}}$, and indicates that GIXR-only 
data analysis is not sensitive to the exact shape of $\delta$-profile in the interface regions. In effect, the same fit goodness can be achieved with a linear or Gaussian interface shape instead of the sinusoidal shape that was used in the analysis. To increase the sensitivity to the interface shape, reflectivity information from a much larger measured angular range is required. For GIXR reflectivity the minimal resolvable feature can be estimated by the formula $\delta z=\lambda / 2 \pi \sin \theta_{\max }$, were $\theta_{\max }$ is the maximal measured angle. For the measurements presented here $\theta_{\max }=5^{\circ}$, therefore the resolution of the optical contrast profile determination is limited by $0.3 \mathrm{~nm}$.

Fig. 2.3a and Table 2.1 show that the addition of EUVR data to the reflectivity analysis does not significantly increase the accuracy of determination of $\Gamma$ and $S_{\Gamma}$. This is primarily because of the high correlations between $S$ and $S_{\Gamma}$ for EUVR data as showed on Fig. 2.4b. However the error of determination $S$ was reduced by a factor two as a result of the simultaneous data analysis of the EUV and X-ray range. Fig. 2.4b shows that the EUVR-only analysis would not provide accurate information about multilayer structure because of the large correlation between parameters. The analysis of correlated errors in simultaneous EUVR and GIXR data analysis showed only a minor decrease of the correlation coefficients as compared to the GIXR-only analysis.

The analysis of correlated errors in simultaneous EUVR and GIXR data analysis showed only a minor decrease of the correlation coefficients as compared to the GIXR-only analysis. However, the simultaneous analysis does significantly increase the accuracy of the determination of the optical constants of the layers in the multilayer structure. According to the Table 2.1, the largest increase of sensitivity was observed for the determination of the density of the LaN layer $\left(\rho_{\mathrm{LaN}}\right)$ and for the determination of the LaN atomic fraction in B layer $\left(\omega_{\mathrm{B}}\right)$. The reason for it is the sensitivity of EUVR data to the optical contrast between spacer and reflector layers in the multilayer. A reduction of the LaN layer density and an increase of the B layer impurity would decrease the optical contrast and result in a decrease of the EUV reflectivity and strong increase of the EUVR $\chi^{2}$. The precise reconstruction of the optical constant profile and especially the optical contrast provides a valuable towards comparing multilayer multilayer mirror deposition processes [2] and towards predicting the reflectivity of multilayerswith different thicknesses or number of periods [3].

\subsection{Conclusions}

In conclusion, a simultaneous analysis of both GIXR and EUVR significantly increases the accuracy of the reconstruction of layer densities and material combination compared to GIXR-only analysis, which will be essential for the use of the reconstructed models for the prediction of EUV reflectivity. The refractive index profiles and their uncertainties can be accurately obtained by GIXR-only data analysis. The addition of EUVR data to the analysis marginally increases the accuracy of the determination of the dimensional parameters. The analysis of correlations indicated that EUVR-only fit will not give accurate representation of multilayer period structure, and therefore can be used only in combination with GIXR. 


\section{References}

[1] S. S. Andreev, M. M. Barysheva, N. I. Chkhalo, S. A. Gusev, A. E. Pestov, V. N. Polkovnikov, D. N. Rogachev, N. N. Salashchenko, Y. A. Vainer, and $\mathrm{S}$. Y. Zuev, Multilayer $\mathrm{X}$-ray mirrors based on $\mathrm{La} / \mathrm{B}_{4} \mathrm{C}$ and $\mathrm{La} / \mathrm{B}_{9} \mathrm{C}$, Technical Physics 55, 1168 (2010).

[2] I. A. Makhotkin, E. Zoethout, R. van de Kruijs, S. N. Yakunin, E. Louis, A. M. Yakunin, V. Banine, S. Müllender, and F. Bijkerk, Short period La/B and LaN/B multilayer mirrors for $6.8 \mathrm{~nm}$ wavelength, Opt. Express 21, 29894 (2013).

[3] I. A. Makhotkin, R. W. E. van de Kruijs, E. Zoethout, E. Louis, and F. Bijkerk, Optimization of LaN/B multilayer mirrors for 6.x $\mathrm{nm}$ wavelength, Proc.SPIE 8848, 8848 (2013).

[4] M. Born and E. Wolf, Principles of optics: electromagnetic theory of propagation, interference and diffraction of light (Elsevier, 2013).

[5] J. Daillant and A. Gibaud, X-ray and neutron reflectivity: principles and applications, Vol. 770 (Springer, 2008).

[6] L. D. Landau, L. P. Pitaevskii, and E. Lifshitz, Electrodynamics of Continuous Media: Volume 8 (Course of Theoretical Physics) (Butterworth-Heinemann, 1984).

[7] V. Kohn, On the theory of reflectivity by an X-ray multilayer mirror, physica status solidi (b) 187, 61 (1995).

[8] K. Stoev and K. Sakurai, Recent theoretical models in grazing incidence X-ray reflectometry, The Rigaku Journal 14, 22 (1997).

[9] B. L. Henke, E. M. Gullikson, and J. C. Davis, X-ray interactions: photoabsorption, scattering, transmission, and reflection at $E=50-30000 \mathrm{eV}, Z=1-92$, Atomic data and nuclear data tables 54, 181 (1993).

[10] V. G. Kon, On the theory of X-ray reflection with multilayer mirrors. DebyeWaller and Nevot-Croce approximations for taking into account the roughness of interfaces (in russian), Journal of Surface Investigation. X-Ray, Synchrotron and Neutron Techniques, 23 (2003).

[11] A. Gibaud and S. Hazra, X-ray reflectivity and diffuse scattering, Current Science 78, 1467 (2000).

[12] I. Hughes and T. Hase, Measurements and their uncertainties: a practical guide to modern error analysis (Oxford University Press, 2010).

[13] A. M. Afanas'ev and M. A. Chuev, Discrete forms of mossbauer spectra, Journal of Experimental and Theoretical Physics 80, 560 (1995). 
[14] G. A. Korn and T. M. Korn, Mathematical handbook for scientists and engineers: definitions, theorems, and formulas for reference and review (Courier Corporation, 2000).

[15] M. Chuev, On correlation analysis and uncertainties estimation of permittivity profiles, personal communication (2013).

[16] T. Tsarfati, R. W. E. van de Kruijs, E. Zoethout, E. Louis, and F. Bijkerk, Nitridation and contrast of $B_{4} C / L a$ interfaces and $X$-ray multilayer optics, Thin Solid Films 518, 7249 (2010).

[17] F. Scholze, J. T. mmler, and G. Ulm, High-accuracy radiometry in the EUV range at the PTB soft X-ray beamline, Metrologia 40, S224 (2003).

[18] J. Tummler, H. Blume, G. Brandt, J. Eden, B. Meyer, H. Scherr, F. Scholz, F. Scholze, and G. Ulm, Characterization of the PTB EUV reflectometry facility for large EUVL optical components, Proc.SPIE 5037, 5037 (2003).

[19] F. Scholze, C. Laubis, C. Buchholz, A. Fischer, A. Kampe, S. Plöger, F. Scholz, and G. Ulm, Polarization dependence of multilayer reflectance in the EUV spectral range, Proc.SPIE 6517, 6517 (2007).

[20] I. A. Makhotkin, E. Zoethout, E. Louis, A. M. Yakunin, S. Müllender, and F. Bijkerk, Spectral properties of La/B - based multilayer mirrors near the boron K absorption edge, Opt. Express 20, 11778 (2012).

[21] M. Fernández-Perea, J. I. Larruquert, J. A. Aznárez, J. A. Méndez, M. VidalDasilva, E. Gullikson, A. Aquila, R. Soufli, and J. L. G. Fierro, Optical constants of electron-beam evaporated boron films in the 6.8-900 eV photon energy range, J. Opt. Soc. Am. A 24, 3800 (2007). 



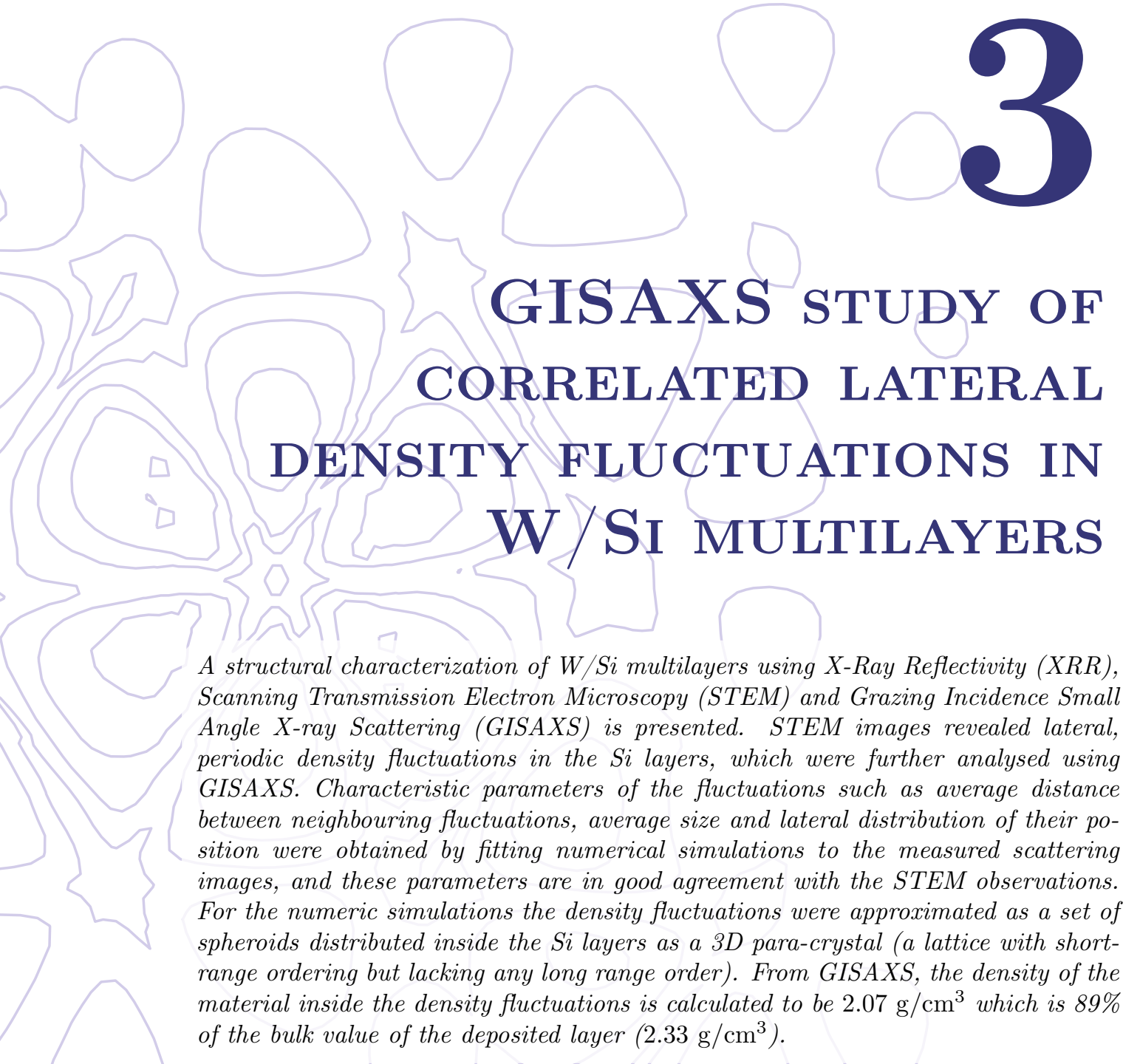




\subsection{Introduction}

Thin film periodic multilayer reflective coatings are used in spectroscopy and optical instruments in the photon energy range of few tens of $\mathrm{eV}$ up to few $\mathrm{keV}$, i.e. from the XUV to the soft X-ray range [1, 2]. Depending on the application, various aspects of the structural quality of reflective coatings should be prioritized during the coating development process. As an example, often the reflectivity should be maximized over a certain bandwidth around a particular wavelength. However, for some applications like X-ray focusing optics the minimization of the off specular diffuse scattering can be more important than maximization of the reflectivity. Primarily, diffuse scattering in multilayers is caused by interface roughness. In specific cases, diffuse scattering in multilayers may also be caused by a $3 \mathrm{D}$ distribution of defects in the volume of the structure [3]. Analysis of the diffuse scattering provides information about the growth process including defects [4]. Such information can indicate directions for further optimization of the reflective properties of the multilayers.

In this study we focus on the characterization of the structural imperfections of a $\mathrm{W} / \mathrm{Si}$ periodic multilayer with a $4.5 \mathrm{~nm}$ period. Such a multilayer is typically being used for fluorescent spectroscopy analysis in the XUV range. A first multilayer structure characterization using Scanning Transmission Electron Microscopy (STEM) suggested that the Si spacer layers have lateral density fluctuations, as opposed to what was expected from the growth model [5]. This observation was confirmed using Grazing Incidence Small Angle X-ray Scattering (GISAXS). Numeric simulations of the GISAXS experimental data were performed to analyse the statistics of the distribution of the density fluctuations and the morphology of the interface roughness.

\subsection{Sample preparation and characterization}

\subsubsection{Sample preparation}

$\mathrm{W} / \mathrm{Si}$ multilayers were deposited on Si superpolished substrates using a magnetron sputtering system [2]. A $6.35 \mathrm{~mm}$ thick substrate was used in order to avoid a deformation (curvature) of the substrate caused by the tensile stress in the multilayer. A W target of $99.95 \%$ and Si target of $99.99 \%$ purity were used. Ar was used as a sputtering gas at a base pressure of $10^{-4}$ mbar. Magnetrons were set at a constant power mode equal to $86.7 \mathrm{~W}$ and $213 \mathrm{~W}$ for the $\mathrm{W}$ and Si targets respectively. The multilayer coating prepared contained $50 \mathrm{~W} / \mathrm{Si}$ bi-layers with a period thickness $D=4.5 \mathrm{~nm}$ and $\mathrm{W}$ layer-thickness to period-thickness ratio of $\Gamma=0.23$.

\subsubsection{Preliminary sample characterization with XRR and HAADF-STEM}

As a post-growth characterization of the manufactured sample, X-ray reflectivity (XRR) was used. The XRR curve was measured on an Empyrean laboratory diffractometer from Malvern Panalytical using characteristic $\mathrm{CuK}_{\alpha 1}$ radiation from a long fine focus line source. Monochromatization and primary collimation of the incident 


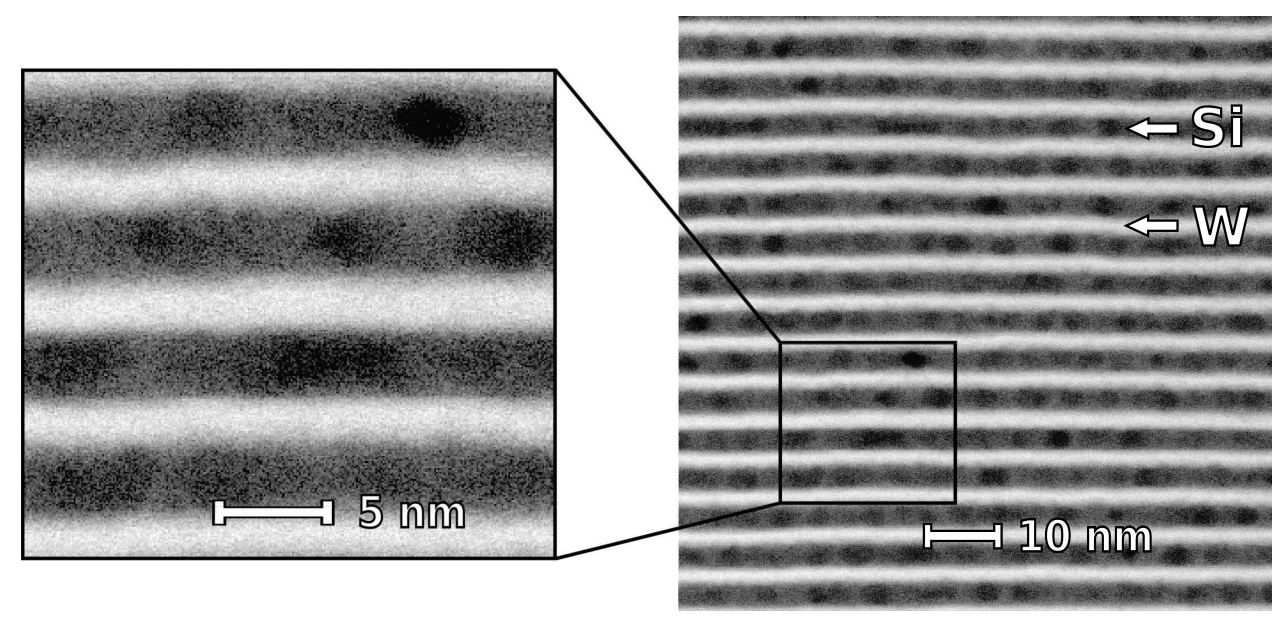

Figure 3.1: HAADF-STEM image of W/Si multilayer. The W layers are shown as brighter areas and $\mathrm{Si}$ as darker areas.

beam was achieved using a four bounce asymmetrically cut germanium monochromator, which yields a beam divergence of $0.012^{\circ}$. Collimation at the detector side was achieved by an antiscatter slit in combination with a tunable electronic receiving slit of a PIXcel3D detector. XRR data was analysed using the assumptionindependent approach [6]. The resulting electron density depth profile was used to verify initial estimations of $D$ and $\Gamma$ resulting in: $D=4.46 \mathrm{~nm}$ and $\Gamma=0.2$. The reconstructed parameters differ slightly from the design values because of the interdiffusion between $\mathrm{W}$ and Si occurring during the deposition. These parameters are used further for the GISAXS numerical simulations presented in Section 3.4 for the qualitative investigation of the structure of the Si layer.

STEM measurements were performed in a Titan 80-300 equipped with a spherical aberration corrector (probe corrector), using an acceleration voltage of $300 \mathrm{kV}$. The microscope was equipped with an energy-dispersive X-ray $\mathrm{Si}(\mathrm{Li})$ spectrometer EDAX; high angle annular dark field electron detector Fischione and Gatan image filter. High resolution bright field TEM measurements and electron diffraction pattern (not shown here) indicated the presence of $\mathrm{W}$ nano-crystals in the $\mathrm{W}$ layers, with a crystallite size comparable to the $\mathrm{W}$ layer thickness, typical for nanometer thickness metal layers grown by sputter deposition. To study the microstructure of the Si layers, high angle annular dark field scanning transmission electrom microscopy (HAADF-STEM) measurements were performed. The HAADF-STEM images (see Fig. 3.1) revealed the presence of inhomogeneities inside the Si layers. Such inhomogeinities are unexpected, since to our knowledge amorphous Si is not known to develop any such density fluctuations during growth.

In Fig. 3.1 Si layers appear darker than the W layers due to both atomic number contrast in HAADF-STEM and thickness effects as considered in [7]. The inhomogeneities inside the Si layers appear to be quasi-periodic, and their distribution is statistically analysed in Appendix 3.6.C. The HAADF-STEM image however does not provide more detailed information on the density fluctuations including their 
positional correlations throughout the multilayer. To extract such information, a GISAXS study was performed, since GISAXS is highly sensitive to correlated structural imperfections (see comprehensive review in [8]).

\subsubsection{GISAXS experiment}

GISAXS measurements were done on the bending magnet beamline Langmuir of the synchrotron radiation source Siberia-2 at the Kurchatov Institute [9]. Monochromatization at the beamline is carried out by a thermally stabilized two bounce $\mathrm{Si}$ monochromator with (111) reflection. Higher harmonics of the monochromatized beam are suppressed with quartz and tungsten X-ray mirrors. The synchrotron beam was collimated with three sets of slits. The resulting beam size is $50 \times 300 \mu \mathrm{m}^{2}$ and the corresponding average direct beam intensity is approximately $3 \cdot 10^{7}$ c.p.s. The vertical beam divergence is 4 arcsec and 20 arcsec horizontally.

Experimental data was measured with a Pilatus100k 2D detector. Measurements were taken at the wavelength $\lambda=0.1 \mathrm{~nm}$ in 12 exposures of 15 minutes each, in order to avoid detector saturation. The angle of incidence was set to $\theta_{0}=0.4^{\circ}$ in between total external reflection and the first Bragg peak. The sum of 12 GISAXS measurements is shown in Fig. 3.2 at a logarithmic scale, where the colorscale of Fig. $3.2 \mathrm{a}$ is chosen to emphasize high intensity scattering, whereas the same data is given in Fig. 3.2b with the colorscale adjusted to emphasize lower intensity details.

The most pronounced feature in the GISAXS scattering are the resonant diffuse scattering sheets [10]. The series of these sheets is marked by arrows 1 in Fig. 3.2a. These sheets are caused by the correlated interface roughness [11]. In literature, resonant diffuse scattering sheets are also being referred to as Bragg sheets [12]. Scattering geometry for the Bragg sheets is shown in Fig. 3.2a'. Here, $\mathbf{k}_{\mathrm{in}}, \mathbf{k}_{\mathrm{sc}}$ are the wave vectors for incidence and scattered beam respectively and $\mathbf{q}=\mathbf{k}_{\mathrm{sc}}-\mathbf{k}_{\mathrm{in}}$ is the reciprocal space vector. Bragg sheets are located at $q_{z} \approx 2 \pi m / D$.

A second feature marked by arrows 2 in Fig. 3.2a is known in literature as the Bragg singularity lines [13]. These Bragg singularity lines are minima caused by the distructive interference of the scattered waves with each other $\left(\mathbf{k}_{\mathrm{sc}}\right.$ and $\mathbf{k}_{\mathrm{sc}}^{\prime}$ in Fig. 3.2a $\mathrm{a}^{\prime \prime}$, while Bragg sheets are due to the Bragg interference of incident and scattering waves. Bragg singularity lines are located at positions for which exit angle of scattered beam $\theta_{\mathrm{sc}}$ satisfies Bragg condition $2 D \sin \left(\theta_{\mathrm{sc}}\right)=m \lambda$ for the multilayer structure.

In addition to the features discussed above one can observe two other features in Fig. 3.2b. The first feature is the "halo" in between Bragg sheets (marked with arrows 3 in Fig. 3.2b. The second feature is the "shadow" in between the third and the fourth Bragg sheets (marked with arrows 4 in Fig. 3.2b). In Section 3.4 we will show that these "halo" and "shadow" features can be reproduced by simulations taking scattering on correlated density fluctuations of the material in the Si layers into account. Within this approach the position of the "shadow" is due to the size of the density fluctuations and the "halo" is due to the correlation in the positions of fluctuations. 

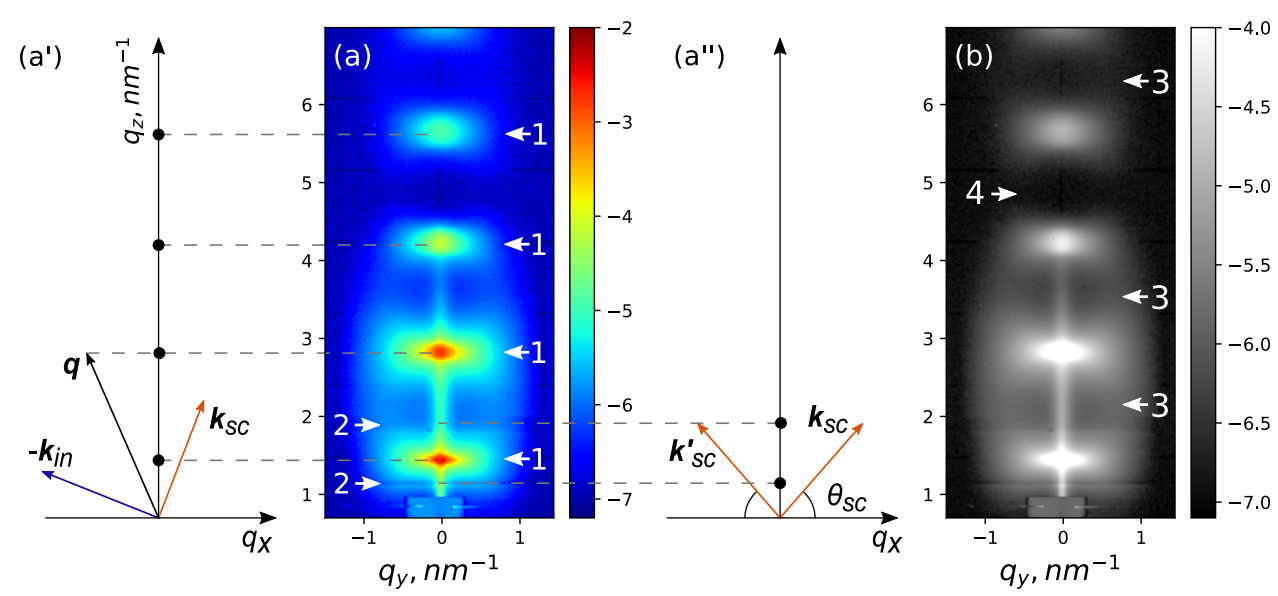

Figure 3.2: a) Experimentally measured GISAXS intensity in arbitrary units in logarithmic scale. b) Same experimental data, though the color scheme and color depth is chosen to emphasize low intensity features. $\mathrm{a}^{\prime}$ ) Scattering geometry for the Bragg sheets. $\mathrm{a}^{\prime \prime}$ ) Scattering geometry for the Bragg singularity lines.

\subsection{GISAXS theoretical background}

Calculations of diffuse X-ray scattering are performed using a perturbation theory [14]. A rigorous formulation of the second order perturbation theory is given in [10]. There, the theory is formulated in terms of the reciprocity theorem of electrodynamics [15]. In this theorem, the scattering length $f$ of the diffuse scattering wave $E_{\text {diff }}=f / r \exp (-i k r)$ has the form:

$$
f=\frac{k_{0}^{2}}{4 \pi} \int E_{\mathrm{in}}(\mathbf{r}) E_{\mathrm{sc}}(\mathbf{r}) \delta \chi(\mathbf{r}) d^{3} V,
$$

where the function $\delta \chi(\mathbf{r})=\chi(\mathbf{r})-\chi_{0}$ represents a deviation of the actual dielectric susceptibility $\chi(\mathbf{r})$ of a structure from the value $\chi_{0}$ of an ideal structure. Eq. 3.1 is written in a scalar approximation. This $\chi_{0}$ value is used in the calculation of the wave fields in the structure: the field $E_{\text {in }}$ is induced by the incident beam, and the field $E_{\mathrm{sc}}$ by the diffusely scattered wave. Eq. 3.1 is commonly referred to as the distorted wave Born approximation (DWBA). We have considered here s-polarized radiation, as this was used in the measurements. Therefore, the fields $E_{\mathrm{in}}$ and $E_{\mathrm{sc}}$ are represented as scalar functions.

The intensity of the diffuse scattering is described with the scattering differential cross section:

$$
\frac{d \sigma}{d \Omega}=\left\langle|f|^{2}\right\rangle .
$$

Here averaging is applied to account for the random nature of imperfections $\delta \chi(\mathbf{r})$ of the structure. $\delta \chi(\mathbf{r})$ is considered to be a stochastic variable, describing a spatial distribution and a structure of the imperfections. In this work we will consider the form of Eq. 3.2 explicitly written for interface roughness (in Section 3.3.1) and 3D paracrystal of the density fluctuations (in Section 3.3.2). Finally, the wave fields 
$E_{\text {in,sc }}$ are considered as plane waves with phase terms $\exp \left( \pm i \mathbf{k}_{\mathrm{in}, \mathrm{sc}} \cdot \mathbf{r}\right)$. Therefore, considering the integration in Eq. 3.1 it is convenient to represent the imperfection of the structure in the form of a Fourier transform: $\delta \hat{\chi}(\mathbf{q})$, where $\mathbf{q}=\mathbf{k}_{\mathrm{sc}}-\mathbf{k}_{\mathrm{in}}$. The way of defining the probability density function of $\delta \hat{\chi}(\mathbf{q})$ defines a recipe for the simulation of the diffuse scattering on various types of imperfections. We now briefly review the theoretical models.

\subsubsection{Scattering on correlated interface roughness}

For the calculation of the diffuse scattering on interface roughness of the multilayer, the wave field within the $j$-th layer is considered to be $E^{(j)}(z)=E_{t}^{(j)} \exp \left(i k_{z} z\right)+$ $E_{r}^{(j)} \exp \left(-i k_{z} z\right)$. Amplitudes of transmitted $E_{t}^{(j)}$ and reflected $E_{r}^{(j)}$ components of the standing wave can be calculated based on a model of the layered structure reconstructed from XRR measurements (see [16] among others). In reference [17], the integration for the scattering length in DWBA (similar to Eq. 1) was done, considering the wave-fields described above, resulting in:

$$
\frac{d \sigma}{d \Omega}=\frac{k_{0}^{4}}{16 \pi^{2}} \sum_{j, k} \sum_{\substack{l, m, n, p \\ \in\{r, t\}}} E_{l, \mathrm{in}}^{(j)} E_{m, \mathrm{sc}}^{(j)} E_{n, \mathrm{in}}^{*(k)} E_{p, \mathrm{sc}}^{*(k)} C_{j k}(\mathbf{q})
$$

The second summation in Eq. 3.3, where the indices $l, m, n, p$ denote the transmitted $(t)$ and reflected $(r)$ waves, has sixteen terms. Each term has a correlation function $C_{j k}(\mathbf{q})=\left\langle\delta \hat{\chi}_{j}(\mathbf{q}) \delta \hat{\chi}_{k}^{*}(\mathbf{q})\right\rangle$ that describes the interface roughness morphology [5] of each $j, k$ pair of interfaces.

In our numeric simulations, we employ Ming's model [18] for the calculation. For the characterization of roughness morphology, Ming's model involves the following parameters: r.m.s. roughness amplitude $\sigma$; lateral correlation length $\xi$; Hurst parameter $H$ that characterizes the jaggedness of a sample and the vertical correlation length $L_{\text {vert }}$. For the detailed description of these parameters, see [4, 18].

\subsubsection{Scattering on the density fluctuations}

Here we consider lateral density fluctuations as an array of spheroids included into a homogeneous matrix of the Si spacer layer. In the STEM image (see Fig. 3.1), one can note that the fluctuations are confined within the Si layers. We note that the vertical sizes are close to the value of the Si layer thickness and are not correlated with the positions along the vertical direction. One can also note that the density fluctuations appear as spheroids of comparable sizes. Additionally, we assume that the density fluctuations are isotropically arranged in the lateral plane inside the $\mathrm{Si}$ layers and we neglect correlations between their sizes and positions. Based on these considerations, the density fluctuations are best described using a 3D para-crystal model employing the mono-dispersion and decoupling approximations.

A comprehensive model for the simulation of diffuse scattering on a 3D paracrystal [19] is given in [20], where scattering from quantum-dots is investigated. There, an expression for the differential cross-section is derived using a decoupling approximation. In addition to the decoupling approximation we simplify the ex- 
pression for the differential cross-section given in [20] assuming the mono-dispersion approximation:

$$
\frac{d \sigma}{d \Omega}=\frac{k_{0}^{4}}{16 \pi^{2}}\left|t_{i} t_{f}\right|^{2}|\Delta \chi|^{2}|\hat{F}(\mathbf{q})|^{2} G(\mathbf{q}) .
$$

That equation is derived assuming:

$$
\delta \chi(\mathbf{r})=\Delta \chi \sum_{\mathbf{R}} F_{\mathbf{R}}(\mathbf{r}) .
$$

Here $F_{\mathbf{R}}(\mathbf{r})$ is the shape function of the density fluctuation. It is equal to unity inside the density fluctuation located at $\mathbf{R}$ and equal to zero elswhere.

Taking the Fourier transform of Eq. 3.5 in Eq. 3.1 results in two functions: $\hat{F}(\mathbf{q})$ and $G(\mathbf{q})$. Form factor function $\hat{F}(\mathbf{q})$ is a Fourier transform of a single density fluctuation shape function $F_{\mathbf{R}}(\mathbf{r})$ located at the origin $\mathbf{R}=0$. This function is deterministic due to the mono-dispersion approximation. We considered a spheroid shape of the density fluctution which has a form factor [21] that can be approximated by:

$$
\hat{F}(\mathbf{q})=4 \pi V_{\mathrm{sp}} \frac{\sin \phi-\phi \cos \phi}{\phi^{3}},
$$

where $\phi=q_{\|} d_{L}+q_{z} d_{z}, d_{L}$ and $d_{z}$ are lateral diameter and height of the spheroid respectively and $V_{\mathrm{sp}}$ is the spheroid volume.

The correlation function $G(\mathbf{q})$ is a sum of phase displacements related to positions of the density fluctuations:

$$
G(\mathbf{q})=\left\langle\sum_{\mathbf{R}, \mathbf{R}^{\prime}} \exp \left(-i\left\{\mathbf{q} \cdot \mathbf{R}-\mathbf{q}^{*} \cdot \mathbf{R}^{\prime}\right\}\right)\right\rangle .
$$

Explicit mathematical expressions for this model are given in Appendix 3.6.A. The characteristic parameters of this model are: lateral mean distance $a_{L}$ between each two neighbouring density fluctuations, dispersion $\sigma_{L}$ of the lateral mean distance, vertical mean distance $a_{z}$ and dispersion $\sigma_{z}$ defined analogously to the lateral parameters, lateral $d_{L}$ and vertical $d_{z}$ sizes of the density fluctuations. Thus, parameters $a_{L}, a_{z}, \sigma_{L}, \sigma_{z}$ describe positions of the density fluctuations and $d_{L}, d_{z}$ describe the size of the density fluctuations.

\subsection{Results and discussion}

\subsubsection{Analysis of correlated interface roughness}

The diffuse scattering due to interface roughness was analysed by fitting model simulations, as described in Section 3.3.1 to three line-extractions of the data: the first line-extraction is taken in the plane of incidence (along $q_{z}$ direction in $q_{y}=$ $0 \mathrm{~nm}^{-1}$ ), while the second and third line-extractions are in the Bragg sheet planes (along $q_{y}$ in $q_{z}=1.5 \mathrm{~nm}^{-1}$ and $q_{z}=2.9 \mathrm{~nm}^{-1}$ ). Experimental data and best fits are shown in Fig. 3.3.

The best-fit parameters are: Hurst parameter $H=1$, roughness r.m.s. amplitude $\sigma=0.2 \mathrm{~nm}$, vertical correlation length $L_{\mathrm{vert}}=48.3 \mathrm{D}$ and lateral correlation 

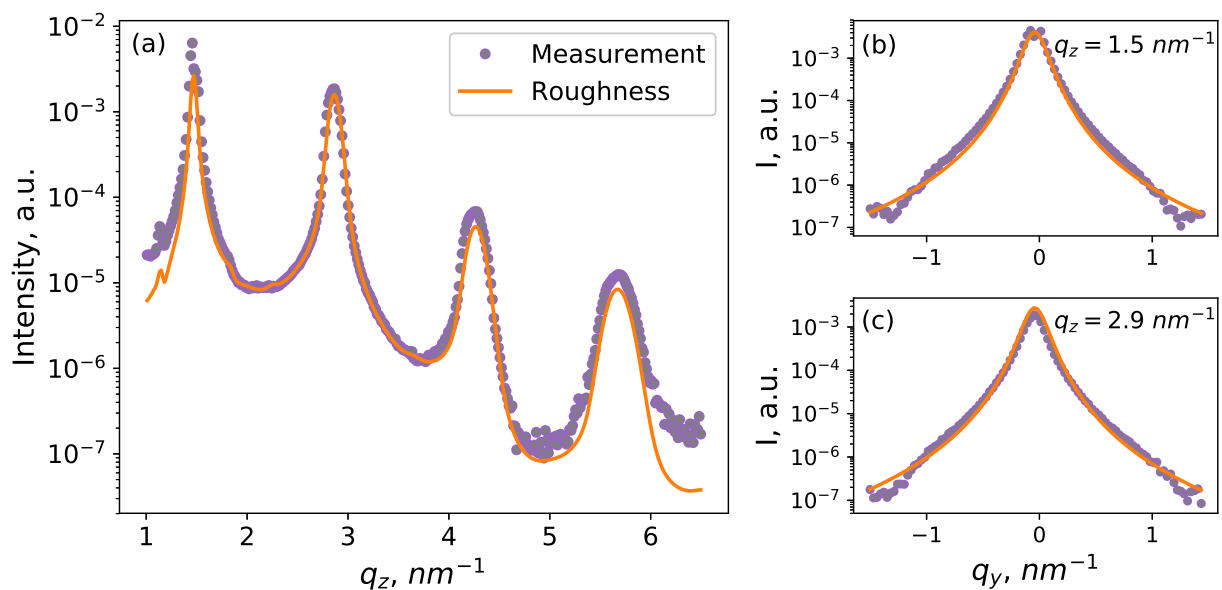

Figure 3.3: Experimental data (markers) and model simulations based on interface roughness (solid line), for line-extractions of data at (a) $q_{y}=0 \mathrm{~nm}^{-1}$, (b) $q_{z}=1.5 \mathrm{~nm}^{-1}$, (c) $q_{z}=2.9 \mathrm{~nm}^{-1}$.

length $\xi=8 \mathrm{~nm}$. From the good agreement between simulations of the interface roughness and the experimental data, we conclude that the diffuse scattering along the plane of incidence Fig. 3.3a and in the Bragg sheets Fig. 3.3b,c is primarily caused by interface roughness, which is well described by Ming's model. The interface roughness at $\sigma=0.2 \mathrm{~nm}$ is considered small. The value of $H=1$ suggests that interfaces are smooth with no jaggedness. The vertical correlation length $\left(L_{\mathrm{vert}}=48.3 D\right)$ is close to the full stack thickness, indicating a high degree of roughness replication from interface to interface.

The obtained parameters allowed us to simulate the scattering caused by the interfaces for the full range of experimental values of $q_{y}, q_{z}$. The results are shown in Fig. 3.4a. As a visual aid, Fig. 3.4b shows the experimental data, together with a contour line based on the simulations of interface roughness scattering that is shown in Fig. 3.4a. We attribute the scattering intensity inside this contour line to the correlated interface, and roughness and intensity outside the contour to the density fluctuations.

\subsubsection{Analysis of correlated density fluctuations}

The intensity of the scattering from density fluctuations is strongly depending on the correlation function and the form factor that describe the distribution of the fluctuations. To build the initial model for fitting of the cross sections one can already assume the characteristic parameters of the density fluctuations distribution.

In the approximation of an ideally ordered distribution of density fluctuations (described in Appendix 3.6.A), the scattering will have a peak at $q_{y} \approx 2 \pi / a_{L}$. In Fig. 3.5 one can see maxima at $q_{y} \approx \pm 0.8 \mathrm{~nm}^{-1}$. Thus, as an initial guess we assume that the mean lateral distance $a_{L}=8 \mathrm{~nm}$. Tails of the curves in Fig. 3.5 are primarily determined by the form factor (see Appendix 3.6.A). Thus, simulating the 

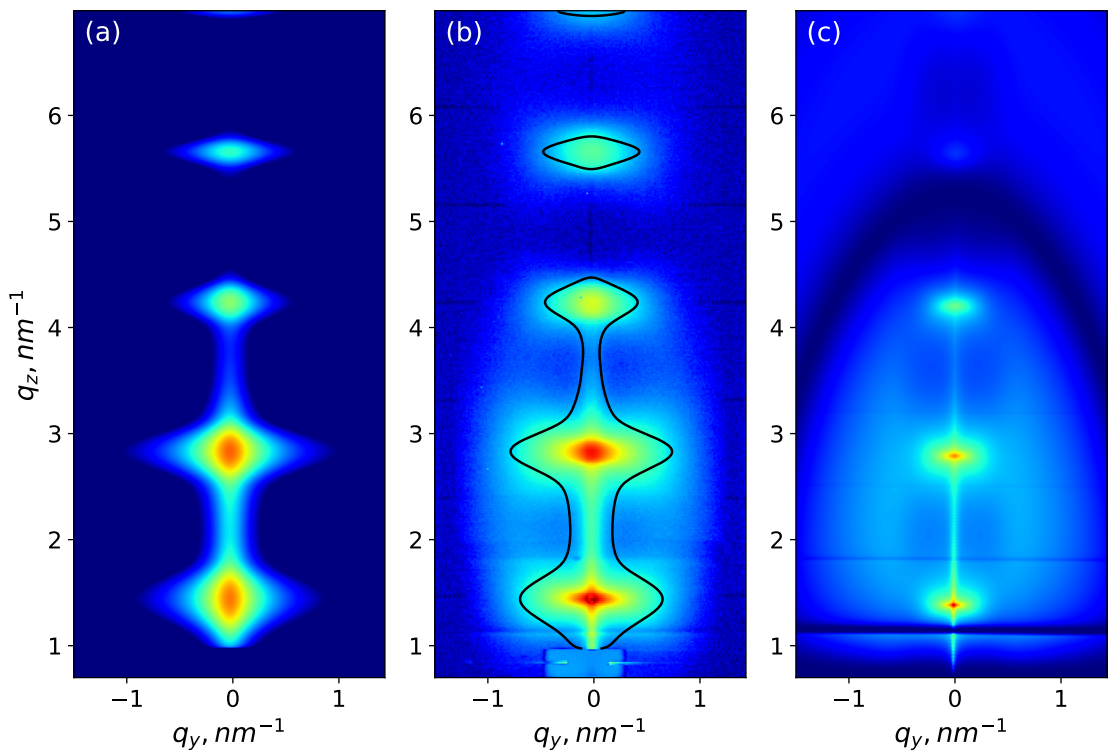

Figure 3.4: GISAXS (a) Simulation of scattering from interface roughness (b) experimental data; black solid contour separates two areas: inner area is mostly affected by interface roughness; the outer area is mostly affected by density fluctuations. (c) Simulation of scattering from density fluctuations inside the Si layers.

slopes of the cross section curves in Fig. 3.5 ( $q_{y}$ range from $0.9 \mathrm{~nm}^{-1}$ untill $1.5 \mathrm{~nm}^{-1}$ ) as an initial guess, we assume the sizes of the density fluctuations $d_{L}=4 \mathrm{~nm}$, $d_{z}=2 \mathrm{~nm}$.

Statistical parameters of the density fluctuations were estimated using a best fit to the GISAXS line-extractions of experimental data. The confidence intervals of these parameters were estimated using a Hessian matrix calculated at the local minima of the best fit. Statistical parameters of the density fluctuations were also estimated using STEM data (see Appendix 3.6.C). The results are shown in Table 3.1.

Visible in Table 3.1, the excellent agreement between STEM and GISAXS in estimates of the positional parameters $\left(a_{L}, a_{z}, \sigma_{L}\right.$ and $\left.\sigma_{z}\right)$. It is noted that the mean lateral distance of the density fluctuations matches very well with interface roughness lateral correlation $a_{L} \approx \xi=8 \mathrm{~nm}$. It hints that formation of the density fluctuations affects interface roughness morphology. The mean vertical distance $a_{z}$ matches very well to the period of the multilayer $D=a_{z}=4.5 \pm 0.2 \mathrm{~nm}$ and dispersion of the mean vertical distance $\sigma_{z}$ is lower than the r.m.s. roughness amplitude $\sigma$ estimated by fitting an interface roughness model, $\sigma_{z}<\sigma$ (see Table 3.1). Thus, we conclude that the density fluctuations are confined in the Si layer and do not penetrate into the $\mathrm{W}$ layer. This observation is consistent with the STEM image shown in Fig. 3.1.

Considering the absolute value of the scattered intensity, the size of the density fluctuations and correlated parameters, using Eq. 3.4 we find the density contrast: 

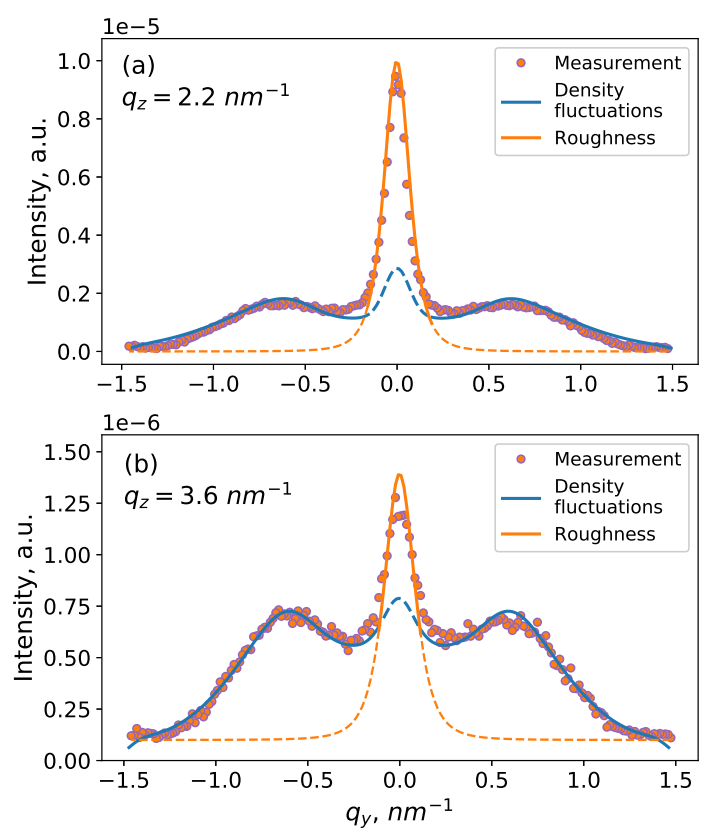

Figure 3.5: Experimental data (markers) and model simulations based on interface roughness (red line) and density fluctuations (blue line), for line-extractions of data at (a) $q_{z}=2.2 \mathrm{~nm}^{-1}$, (b) $q_{z}=3.6 \mathrm{~nm}^{-1}$.

$\Delta \rho=0.26 \pm 0.05 \mathrm{~g} / \mathrm{cm}^{3}$, which is approximately $11 \%$ percent of bulk Si density in normal conditions.

Using the GISAXS best fit parameters, the intensity of the diffuse scattering was simulated for the full area of the 2D detector shown in Fig. 3.4c. In Fig. 3.4c one notes the interesting effect that scattering on the density fluctuations not only causes an enhancement of the scattered intensity between Bragg sheets ("halo"), but also a "shadow" of the scattering by destructive interference. This "shadow" is defined by the shapes of the density fluctuations in the statistical ensemble. The position of the shadow in Fig. 3.4c is consistent with experimental data in Fig. 3.4b. Numerical examples of how "shadow" and "halo" features change with the variation of the parameters of the para-crystal model are discussed in detail in Appendix 3.6.B. The model used for the numerical simulations is one of the simplest to describe the arrangement of the density fluctuations in the periodical structure. However, the good agreement of simulations with measurement data justifies the used approximation.

Comparing the absolute scattered intensities from interface roughness and density fluctuations, we conclude that diffuse scattering in the studied W/Si multilayer system is primarilly due to the interface roughness, with only approximately $8 \%$ of the total scattered intensity being caused by the density fluctuations. The formation of the density fluctuations in the Si layers of a W/Si multilayer system is unexpected. Additionaly, comparison of the mean lateral distance of the density 
Table 3.1: Calculated parameters of the density fluctuations using HAADF-STEM and GISAXS:

\begin{tabular}{c|c|c}
\hline & HAADF-STEM & GISAXS \\
\hline \hline$a_{L}, \mathrm{~nm}$ & $8.1 \pm 0.8$ & $8.0 \pm 0.6$ \\
$\sigma_{L}, \mathrm{~nm}$ & $4 \pm 1$ & $3.2 \pm 0.9$ \\
$a_{z}, \mathrm{~nm}$ & $4.92 \pm 0.09$ & $4.5 \pm 0.2$ \\
$\sigma_{z}, \mathrm{~nm}$ & $0.13 \pm 0.07$ & $0.11 \pm 0.09$ \\
$d_{L}, \mathrm{~nm}$ & $4 \pm 1$ & $4.7 \pm 0.4$ \\
$d_{z}, \mathrm{~nm}$ & $2.2 \pm 0.4$ & $1.7 \pm 0.2$ \\
\hline
\end{tabular}

fluctuations and lateral correlation length of the interface roughness hints that density fluctuations affect the interface roughness morphology. Thus, the analysis of these density fluctuations is of interest for understanding of the multilayer growth model.

Our hypothesis is that the density fluctuations in the Si layers are formed due to the interaction with the high energy back scattered ions present during the magnetron sputtering deposition. Interacting with the sample surface during the $\mathrm{Si}$ layer growth, these high energy ions distribute energy to the Si layers allowing the formation of the lower density phase i.e. density fluctuations. Preliminary analysis of various $\mathrm{W} / \mathrm{Si}$ multilayers deposited with various doses of ion assistance show that higher ion currents result in stronger "shadow" and "halo" effects. For a more detailed investigation of the density fluctuations, the formalism of physical kinetics [22] can be used. That theory allows to analyze the dynamics of statistical parameters of density fluctuations. In this work, we estimated the final statistical parameters of the density fluctuations, which can be used in further analysis in the formalism of physical kinetics as a subject of further research.

\subsection{Conclusions}

HAADF-STEM and GISAXS were used to study density fluctuations inside Si layers in periodic $\mathrm{W} / \mathrm{Si}$ multilayers of nanoscale thickness films. The fluctuations are ordered vertically with a dispersion of $\sigma_{z} \approx 0.11 \mathrm{~nm}$ and mean distance $a_{z} \approx 4.5 \mathrm{~nm}$ which is equal to the period of the multilayer sample. In the lateral direction, i.e. within the Si layer,these density fluctuations have a mean mutual distance of $a_{L} \approx 8 \mathrm{~nm}$, while the dispersion in the lateral direction is $\sigma_{L} \approx 3.2 \mathrm{~nm}$. The density fluctuations are strongly confined within the Si layers and have reduced density $\left(\Delta \rho \approx 0.26 \mathrm{~g} / \mathrm{cm}^{3}\right)$. This study exemplifies the level of detail on growth phenomena that can be found using a combination of STEM and GISAXS analysis.

\subsection{Appendix}

\subsection{A Correlation function of the para-crystal model}

For the simulations in Section 3.4.2, the distribution of the density fluctuations is taken into account using a para-crystal model. Considering the HAADF-STEM 
data (Fig. 3.1) we assume that fluctuations have short range ordering: the positions are correlated stronger for neighboring density fluctuations then for distant density fluctuations. Short range ordering is taken into account using cumulative position errors [20]. Within that approach the correlation function for a $1 \mathrm{D}$ para-crystal lattice has a form:

$$
G(\mathbf{q})=\Phi \gamma+2 \Phi \operatorname{Re}(\nu[\gamma-\tau]),
$$

where $\nu=\xi \eta /\left(\xi \xi^{*}-\xi \eta\right)$ and $\tau=\left(1-(\xi \eta)^{N}\right) /(1-\xi \eta), \xi=\exp (-i \mathbf{q} \cdot \mathbf{a})$ is the phase displacement related to the para-crystal lattice vector $\mathbf{a}, \eta=\langle\exp (\mathbf{q} \cdot \delta)\rangle$ is attributed to the position dispersion, $\delta$ is the cumulative position error vector and $\gamma$ is an effective parameter related to the number of irradiated particles $N$ and X-ray absorption:

$$
\gamma=\frac{1-\Phi(\mathbf{q}, N)}{1-\Phi(\mathbf{q}, 1)} ; \quad \Phi(\mathbf{q}, N)=\exp (-2 N \operatorname{Im}(\mathbf{q} \cdot \mathbf{a})) .
$$

Assuming that the positions of the density fluctuations are normally distributed, $\eta$ can be calculated using a general formula [23]:

$$
\eta=\exp \left\{\left(q_{x} \sigma_{x}\right)^{2} / 2\right\} \exp \left\{\left(q_{y} \sigma_{y}\right)^{2} / 2\right\} \exp \left\{\left(\operatorname{Re}\left(q_{z}\right) \sigma_{z}\right)^{2} / 2\right\}
$$

where $\sigma$ is the vector which components are position dispersion parameters along $x$, $y$, and $z$ axes. In the correlation function Eq. 3.8 absorption of X-rays is taken into account. Note here that Eq. 3.8 takes into account 3D displacement of an object from its original positions defined as a $1 \mathrm{D}$ para-crystal lattice.

Let us now consider limiting cases. In case of no absorption: $\gamma \rightarrow N$, correlation function converges to:

$$
G(\mathbf{q})=N+2 \operatorname{Re}(\nu[N-\tau]) .
$$

Both functions Eq. 3.8 and Eq. 3.11 have limits: $\lim _{|\mathbf{q}| \rightarrow 0} G \rightarrow N^{2}$ and $\lim _{|\mathbf{q}| \rightarrow \infty} G \rightarrow$ $N$. In Eq. 3.4 one can notice that dependence of the differential scattering crosssection on $q_{y}$ is defined with the correlation function and the form factor Eq. 3.6. For $|\mathbf{q}| \rightarrow \infty$ the correlation function is constant. Thus, in this case the scattering intensity is primarily due to the form factor Eq. 3.6. In other words we can assume that shape of the scattering "tales" in line-extractions of the experimental data shown in Fig. 3.5 is solely defined with the form factor. This assumption was used as an initial guess in the fitting procedure in Section 3.4.2. Finally in the limiting case of no dispersion $|\delta| \rightarrow 0$, we obtain:

$$
G=\frac{\sin ^{2}(\mathbf{q} \cdot \mathbf{a} N / 2)}{\sin ^{2}(\mathbf{q} \cdot \mathbf{a} / 2)} .
$$

In this case the correlation function converges to the interference function. In other words within the para-crystal model scattering on an ideal distribution of fluctuations is similar to diffraction from an ideal crystal.

For the numeric simulations in Section 3.4.2 we approximate the correlation function of the $3 \mathrm{D}$ distribution as:

$$
G_{3 \mathrm{D}}(\mathbf{q}) \approx G_{1}(\mathbf{q}) G_{2}(\mathbf{q}) G_{3}(\mathbf{q})
$$


where $G_{i}$ is the correlation function of the $1 \mathrm{D}$ para-crystal model Eq. 3.8, with 3D lattice basis vectors chosen as:

$$
\mathbf{a}_{1}=\left(a_{L}, 0,0\right) ; \quad \mathbf{a}_{2}=\left(0, a_{L}, 0\right) ; \quad \mathbf{a}_{3}=\left(0,0, a_{z}\right),
$$

and the dispersion is parametrized with vectors:

$$
\sigma_{1,2,3}=\left(\sigma_{L}, \sigma_{L}, \sigma_{z}\right)
$$

Strictly speaking, Eq. 3.13 is not taking into account position cross-correlations between different lattice vectors, i.e. displacements of an object along $\mathbf{a}_{i}$ and $\mathbf{a}_{j}$, $i \neq j$ are considered as statistically independent. To compensate for this in the simulations we performed azimuthal averaging of the correlation function: Eq. 3.13 is numerically integrated with respect to rotation of basis vectors around the $z$ axis. In the following sections we discuss how variation of the statistical parameters around the best fit solution affects the correlation function and the form factor.

\subsection{B Variation of statistical parameters}

The correlation function (Eq. 3.13), calculated for the best fit parameters (see Table 3.1) is shown in Fig. 3.6a,b,c and in Fig. 3.6d,e,f for various lateral and vertical mean distance parameters $a_{L}$ and $a_{z}$. Examples of the density fluctuations ensembles were made by Monte Carlo process using the same statistical parameters that were used to calculate the corresponding correlation functions, see Fig. 3.6a'$f^{\prime}$. Blue lines represent $W$ layers, white lines - Si layers. Positions of the density fluctuations are represented schematichally with dots.

It has been shown in Appendix 3.6.A that for the limiting case $|\mathbf{q}| \rightarrow \infty$ the correlation function converges to a constant value. Indeed, in Fig. 3.6 one can see that the correlation function is constant near the edges of the frame. Increasing and decreasing the value of lateral mean distance $a_{L}$ results in a change of the "halo" along the $q_{y}$ axis (see Fig. 3.6a,b,c). The width of the "halo" is proportional to $\sim 2 \pi / a_{L}$. A change of the vertical mean distance $a_{z}$ Fig. 3.6d,e,f results in a shift of Bragg sheets and the "halo" along the $q_{z}$ direction. It is important to note here that Bragg sheets in Fig. 3.6d,e, f are not caused by interface roughness. Here, Bragg sheets are due to the periodicity of the density fluctuations in the $z$ direction. In the experimental data in Fig. 3.4a these peaks are not resolvable due to interface roughness contribution to the diffuse scattering Fig. 3.4b. However, the vertical position of fluctuations can be estimated by the shape of the "halo" feature.

In the HAADF-STEM image Fig. 3.1 one can see that density fluctuations are confined within the Si-spacer layer. The same conclusion can be drawn from best fit parameters: mean vertical distance $a_{z}$ is equal to the period of the multilayer $D=a_{z}=4.5 \mathrm{~nm}$ and dispersion of the mean vertical distance $\sigma_{z}$ is lower than r.m.s. roughness amplitude $\sigma$ estimated by fitting interface roughness model, $\sigma_{z}<\sigma$. Let us consider the case in which fluctuations starts to penetrate $\mathrm{W}$ layer. One can simulate that situation with increasing parameter $\sigma_{z}$. In that case the "halo" features are blurred significantly already at the dispersion values of $\sigma_{z}=0.5 \mathrm{~nm}$ (see Fig. 3.7f). 
(a)

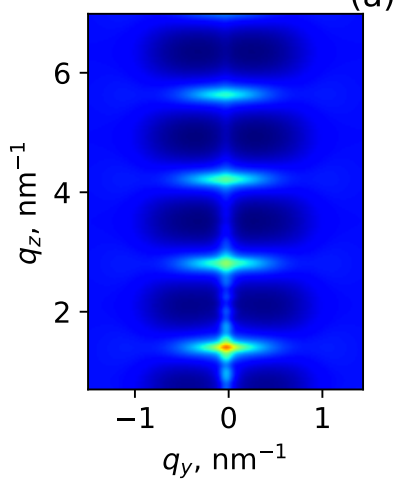

(a')

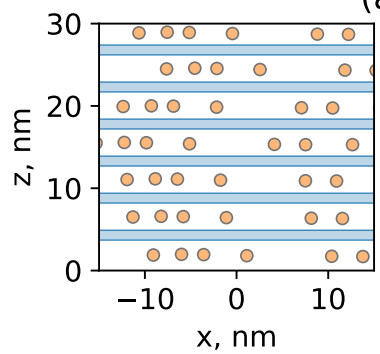

(d)

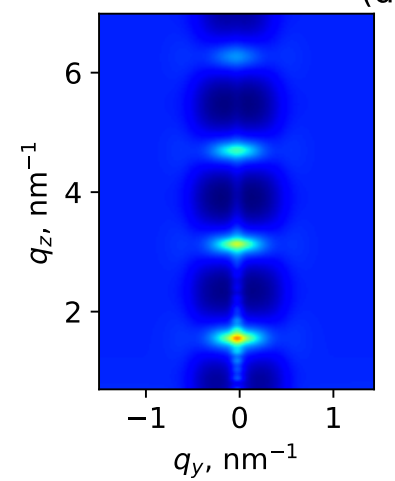

(d')

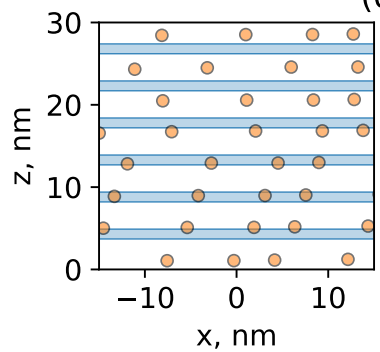

(b)
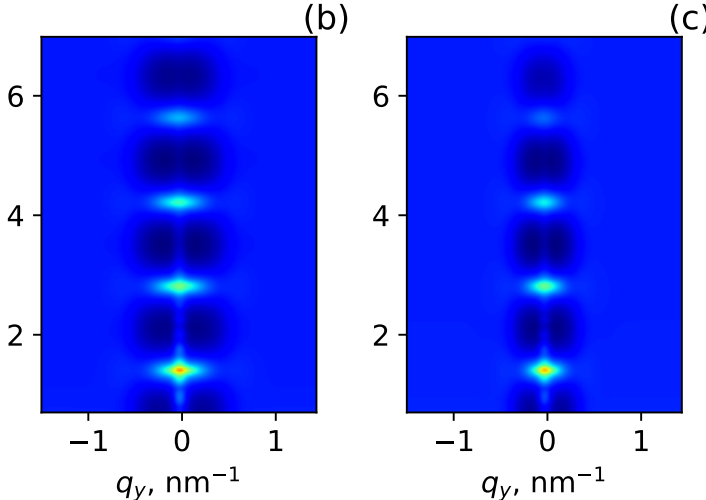

(b')

(c')
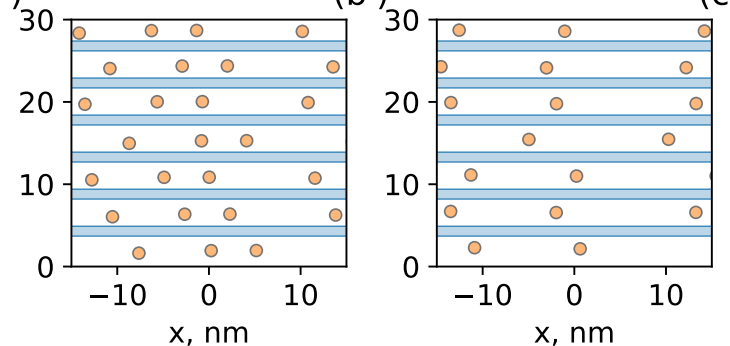

(e)

(f)

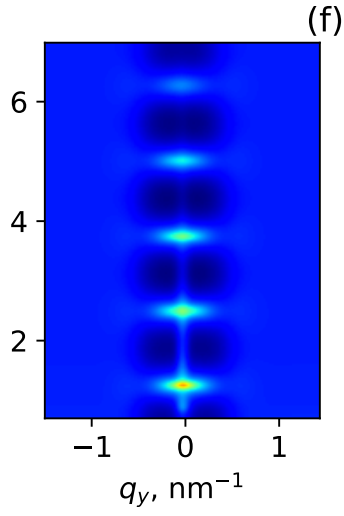

(e')

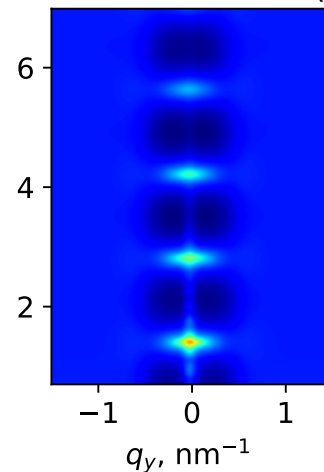

(f')
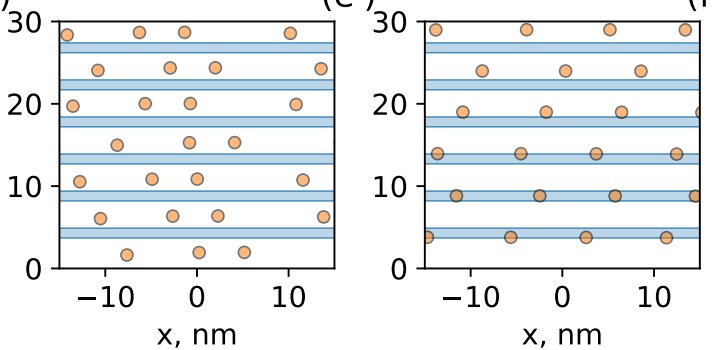

Figure 3.6: Correlation function $|G(\mathbf{q})|$ with various mean lateral distance: (a) $a_{L}=5 \mathrm{~nm}$, (b) $a_{L}=8 \mathrm{~nm}$, (c) $a_{L}=12 \mathrm{~nm}$; and with various mean vertical distance: (d) $a_{z}=4 \mathrm{~nm},(\mathrm{e})$ $a_{z}=4.5 \mathrm{~nm}$, (f) $a_{z}=5 \mathrm{~nm}$. $\left(\mathrm{a}^{\prime}\right)-\left(\mathrm{f}^{\prime}\right)$ Examples of the density fluctuations ensembles that satisfy the corresponding statistical parameters. 
(a)
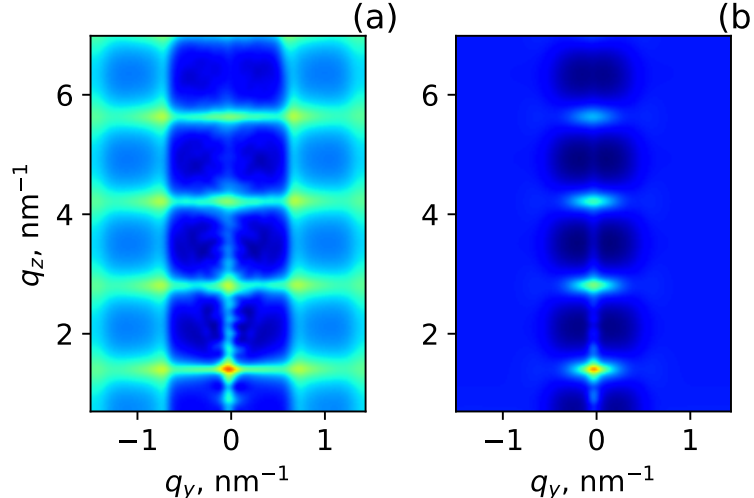

(b)

(c)

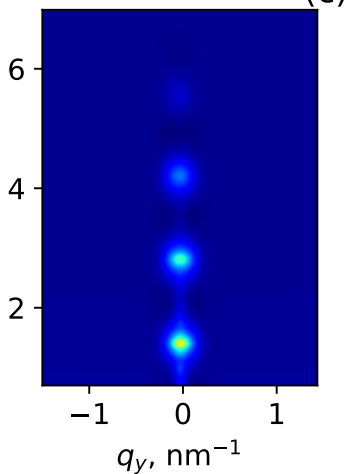

(a')
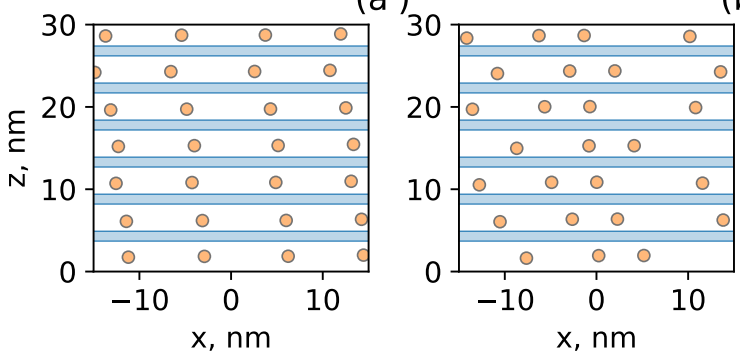

(b')

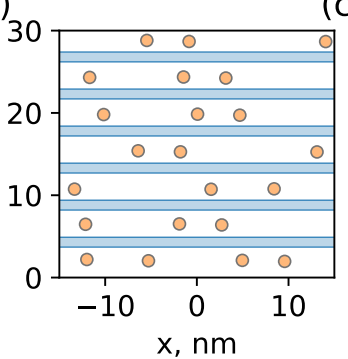

(d)

(e)

(f)
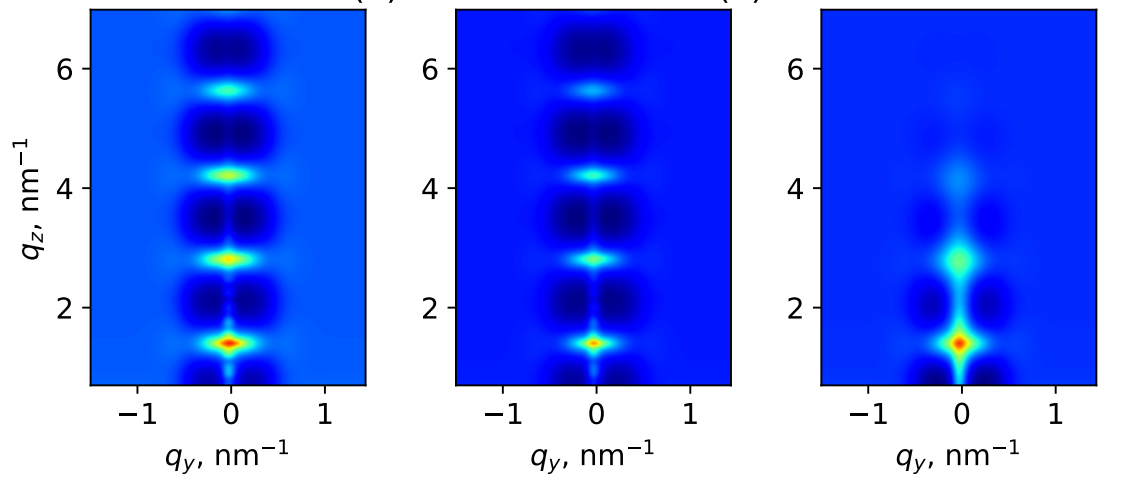

(d')
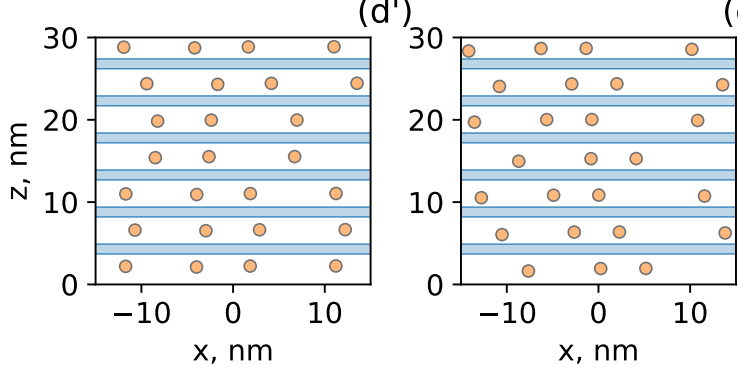

$\left(f^{\prime}\right)$

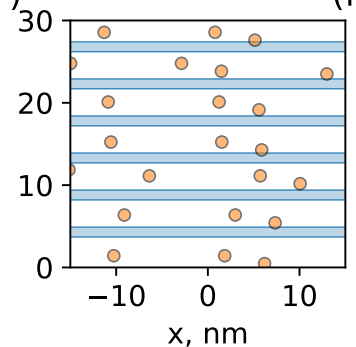

Figure 3.7: Correlation function $|G(\mathbf{q})|$ with various dispersion in lateral distance: (a) $\sigma_{L}=$ $0.8 \mathrm{~nm}$, (b) $\sigma_{L}=3.23 \mathrm{~nm}$, (c) $\sigma_{L}=8 \mathrm{~nm}$; and with various dispersion in vertical distance: (d) $\sigma_{z}=0.05 \mathrm{~nm}$, (e) $\sigma_{z}=0.11 \mathrm{~nm}$, (f) $\sigma_{z}=0.5 \mathrm{~nm}$. ( (a $\left.{ }^{\prime}\right)-\left(\mathrm{f}^{\prime}\right)$ Examples of the density fluctuations ensembles that satisfy the corresponding statistical parameters. 

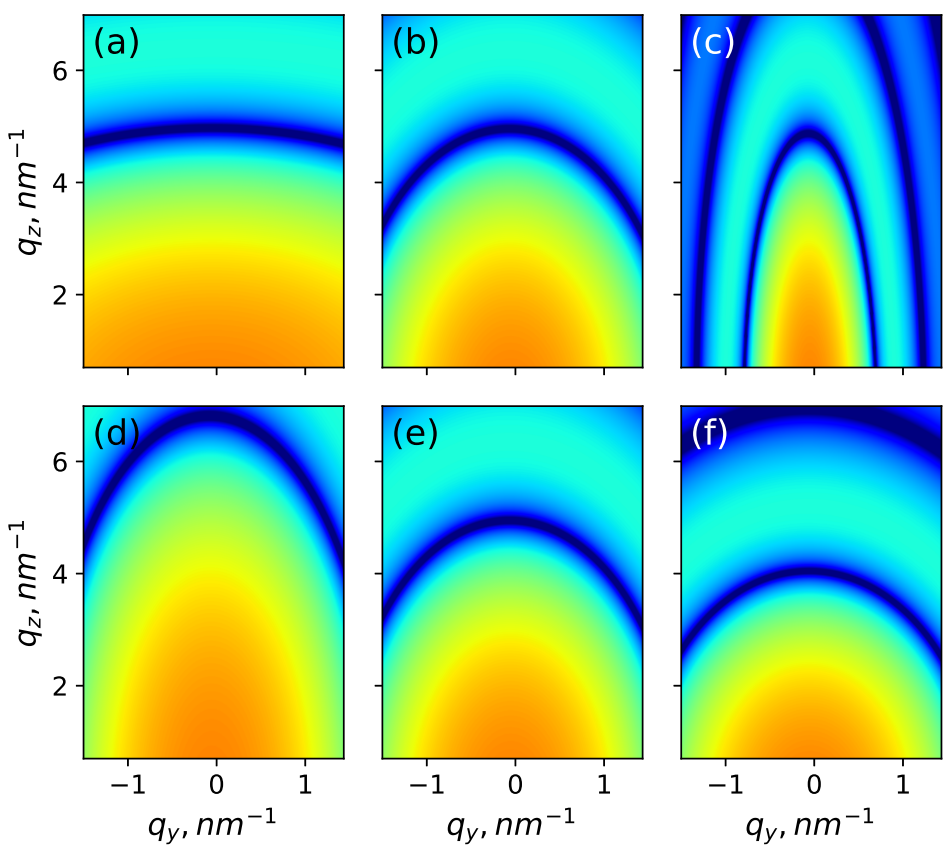

Figure 3.8: Form factor function $|\hat{F}(\mathbf{q})|^{2}$ with various lateral size: (a) $d_{L}=2 \mathrm{~nm}$, (b) $d_{L}=4.7 \mathrm{~nm}$, (c) $d_{L}=12 \mathrm{~nm}$; and with various vertical size: (d) $d_{z}=1.3 \mathrm{~nm}$, (e) $d_{z}=1.7 \mathrm{~nm}$, (f) $d_{z}=2.3 \mathrm{~nm}$.

In the opposite case of low values of distance dispersion $\sigma_{z}=0.11 \mathrm{~nm}$ and $\sigma_{L}=0.8 \mathrm{~nm}$ (see Fig. 3.7a) the correlation function similar to the diffraction on the crystal powder. That observation is consistent with Eq. 3.12 where the limiting case $|\sigma| \rightarrow 0$ is considered.

The form factor function Eq. 3.6 calculated for various size parameters is shown in Fig. 3.8. One can observe in Fig. 3.8 that position of the "shadow" from the best fit model (see Fig. 3.4c) is not as broad as it is in experimental data (see Fig. 3.4a). This might be due to the mono-dispersion approximation. Finally, one can observe in Fig. 3.8 that value of the form factor is decreasing along the $q_{z}$ direction. Since the scattering differential cross-section distribution along $q_{y}$ is defined only with the correlation function and the form factor (see Eq. 3.4) and the correlation function is constant near the edge of the frame in Fig. 3.6, the tails in Fig. 3.5 are defined by the form factor.

\subsection{Statistical analysis of HAADF-STEM image}

In this section we describe procedure that has been employed for the estimation of the statistical parameters in Table 3.1 from STEM image. Boundaries of fluctuations were manually marked on the STEM image four points for each dark object in Fig. 3.9. 


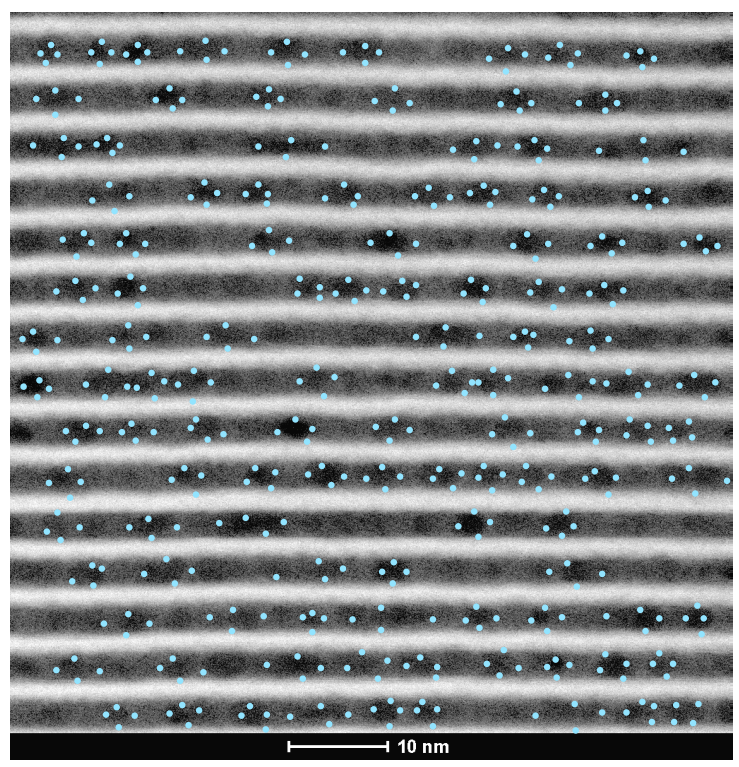

Figure 3.9: HAADF-STEM image of a W/Si multilayer. Each density fluctuation is manually marked with four dots for the statistical analysis.

Position of each fluctuation has been calculated as a center of mass of these four points. Lateral and vertical sizes has been calculated for each fluctuation as $\max \left(x_{i}\right)-\min \left(x_{i}\right)$ and $\max \left(y_{i}\right)-\min \left(y_{i}\right)$ respectively, where $i=1,2,3,4$. Thus statistical parameters has been estimated. To estimate confidence intervals of these parameters we used t-distribution. Position parameters $a_{L}, a_{z}, \sigma_{L}$ and $\sigma_{z}$ were considered to be normally distributed, while size parameters $d_{L}$ and $d_{z}$ were considered to be gamma-distributed, since variation of size parameters are big and negative values are nonphysical. Negative $a_{L}$ and $a_{z}$ parameters are nonphysical as well, however since variation of these parameters is smaller than that of the size parameters, therefore we consider normal distribution to be a valid assumption for these parameters.

\subsection{XRR analysis}

X-ray reflectivity (XRR) curve fitting has been used for a preliminary characterisation of the sample. XRR was measured on a laboratory diffractometer using the characteristic $\mathrm{CuK}_{\alpha}$ radiation (incident beam wavelength $\lambda=0.154 \mathrm{~nm}$ ). The free-form approach were used for XRR fitting, see [6] for detailed description. XRR curve was measured up until incidence angle $\theta_{\max }=8^{\circ}$. That corresponds to the maximal vertical momentum transfer $q_{z}^{(\max )}=11.4 \mathrm{~nm}^{-1}$. According to the sampling theorem, minimal discretisation step of the optical constant profile ( $\delta$-profile) is $d_{\min }=\pi / q_{z}^{(\max )}$. Fitting by a model with discretisation step lower than $d_{\min }$ 
do not provide relevant information about the structure of the sample. Therefore, the model of $\mathrm{W} / \mathrm{Si}$ bi-layer period contained 16 sublayers with individual thickness of approximately $0.28 \mathrm{~nm}$ each. In the model all 50 periods were assumed identical. Measured XRR curve were fitted by simulated curve using the derivative-free bound-constrained optimisation algorithm BOBYGA [24]. The $\chi^{2}$ functional [25] were used as a goodness of fit criterion. The measured XRR curve and the best fit simulated curve are shown in Fig. 3.10a. Residuals normalised on measurement uncertainty are shown in in Fig. 3.10b. The best fit model corresponds to the goodness of fit criterion of $\chi^{2}=2.3$. The $\delta$-profile of the best fit is shown in Fig. 3.10c. Model uncertainties (blue error corridors in Fig. 3.10c were calculated using the formalism of inverse Hessian.

In the model, the surface of the sample was assumed separately from the period to take oxidation into account. That allowed to improve goodness of fit in-between
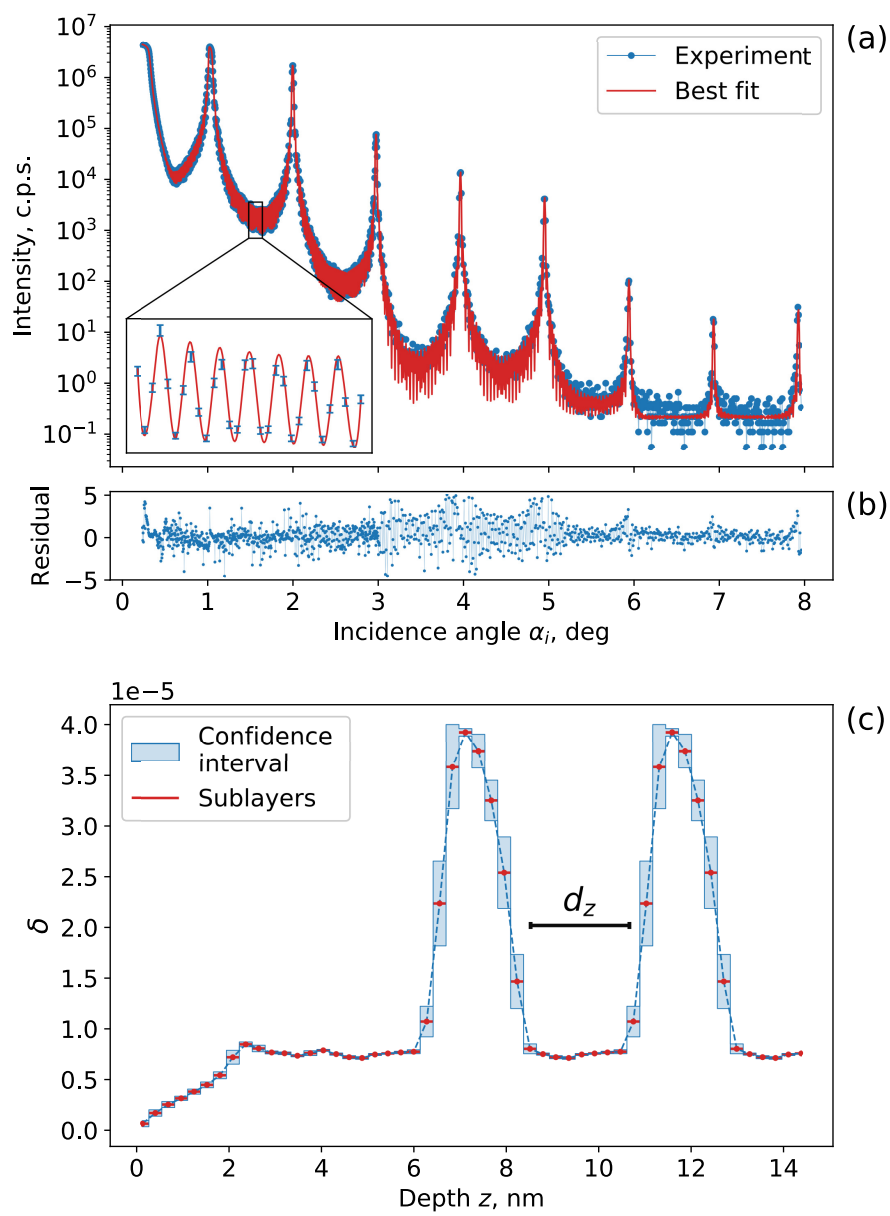

Figure 3.10: XRR analysis. (a) Measured XRR curve and best fit. (b) Residuals. (c) Best fit $\delta$-profile. 
Bragg peaks (see inset in Fig. 3.10a). As a reference the vertical size of the density fluctuations $d_{z}$ is shown in Fig. 3.10c. One can note that vertical size of the density fluctuation fits perfectly in the Si layer, which is consistent with GISAXS and TEM data. 


\section{References}

[1] P. F. Fewster, X-ray analysis of thin films and multilayers, Reports on Progress in Physics 59, 1339 (1996).

[2] E. Louis, A. Yakshin, T. Tsarfati, and F. Bijkerk, Nanometer interface and materials control for multilayer EUV-optical applications, Progress in Surface Science 86, 255 (2011).

[3] U. Pietsch, V. Holý, and T. Baumbach, High-Resolution X-Ray Scattering From Thin Films to Lateral Nanostructures (Springer New York, 2004).

[4] P. Siffalovic, M. Jergel, and E. Majkova, GISAXS-probe of buried interfaces in multilayered thin films, X-ray Scattering (2011).

[5] M. Pelliccione and T.-M. Lu, Evolution of thin film morphology, Vol. 108 (Springer New York, 2008).

[6] A. Zameshin, I. A. Makhotkin, S. N. Yakunin, R. W. E. van de Kruijs, A. E. Yakshin, and F. Bijkerk, Reconstruction of interfaces of periodic multilayers from X-ray reflectivity using a free-form approach, Journal of Applied Crystallography 49, 1300 (2016).

[7] W. Van den Broek, A. Rosenauer, B. Goris, G. Martinez, S. Bals, S. Van Aert, and D. Van Dyck, Correction of non-linear thickness effects in HAADF-STEM electron tomography, Ultramicroscopy 116, 8 (2012).

[8] G. Renaud, R. Lazzari, and F. Leroy, Probing surface and interface morphology with grazing incidence small angle $X$-ray scattering, Surface Science Reports 64, 255 (2009).

[9] V. Korchuganov, A. Belkov, Y. Fomin, E. Kaportsev, G. Kovachev, M. Kovalchuk, Y. Krylov, K. Kuznetsov, V. Kvardakov, V. Leonov, et al., The development of synchrotron radiation source of NRC "Kurchatov Institute", (Russian Particle Accelerator Conference (RuPAC), 2012) pp. 24-28.

[10] V. Kaganer, S. Stepanov, and R. Köhler, Effect of roughness correlations in multilayers on Bragg peaks in X-ray diffuse scattering, Physica B: Condensed Matter 221, 34 (1996).

[11] V. Holỳ and T. Baumbach, Nonspecular X-ray reflection from rough multilayers, Physical Review B 49, 10668 (1994).

[12] P. Siffalovic, E. Majkova, L. Chitu, M. Jergel, S. Luby, J. Keckes, G. Maier, A. Timmann, S. Roth, T. Tsuru, et al., Characterization of Mo/Si soft X-ray multilayer mirrors by grazing-incidence small-angle $X$-ray scattering, Vacuum 84, 19 (2009).

[13] V. Kaganer, S. Stepanov, and R. Köhler, Bragg diffraction peaks in X-ray diffuse scattering from multilayers with rough interfaces, Physical Review B 52, 16369 (1995). 
[14] S. Sinha, E. Sirota, S. Garoff, and H. Stanley, X-ray and neutron scattering from rough surfaces, Physical Review B 38, 2297 (1988).

[15] L. D. Landau, L. P. Pitaevskii, and E. Lifshitz, Electrodynamics of Continuous Media: Volume 8 (Course of Theoretical Physics) (Butterworth-Heinemann, 1984).

[16] S. N. Yakunin, I. A. Makhotkin, K. V. Nikolaev, R. W. E. van de Kruijs, M. A. Chuev, and F. Bijkerk, Combined EUV reflectance and X-ray reflectivity data analysis of periodic multilayer structures, Opt. Express 22, 20076 (2014).

[17] J. Daillant and O. Bélorgey, Surface scattering of X-rays in thin films. Part I. Theoretical treatment, The Journal of chemical physics 97, 5824 (1992).

[18] Z. Ming, A. Krol, Y. Soo, Y. Kao, J. Park, and K. Wang, Microscopic structure of interfaces in $S i_{1-x} G e_{x} /$ Si heterostructures and superlattices studied by $X$-ray scattering and fluorescence yield, Physical Review B 47, 16373 (1993).

[19] J. L. Eads and R. P. Millane, Diffraction by the ideal paracrystal, Acta Crystallographica Section A 57, 507 (2001).

[20] M. Buljan, N. Radić, S. Bernstorff, G. Dražić, I. Bogdanović-Radović, and V. Holỳ, Grazing-incidence small-angle X-ray scattering: application to the study of quantum dot lattices, Acta Crystallographica Section A: Foundations of Crystallography 68, 124 (2012).

[21] R. Lazzari, IsGISAXS: a program for grazing-incidence small-angle X-ray scattering analysis of supported islands, Journal of Applied Crystallography 35, 406 (2002).

[22] L. D. Landau, E. Lifshitz, and L. Pitaevskij, Course of theoretical physics. vol. 10: Physical kinetics (Oxford, 1981).

[23] A. P. Payne and B. M. Clemens, Influence of roughness distributions and correlations on X-ray diffraction from superlattices, Phys. Rev. B 47, 2289 (1993).

[24] M. J. Powell, The BOBYQA algorithm for bound constrained optimization without derivatives, Cambridge NA Report NA2009/06, University of Cambridge, Cambridge , 26 (2009).

[25] I. Hughes and T. Hase, Measurements and their uncertainties: a practical guide to modern error analysis (Oxford University Press, 2010). 




\subsection{Introduction}

The motivation for the characterization of crystal surfaces can be found in, for example, the development of topological insulators [1] and spin-injection structures [2] in which their properties depend on the crystal subsurface structure. However, the characterization of this crystal subsurface is challenging because it typically requires high brilliance synchrotron radiation [3] that is generally not as readily available as radiation from compact lab-scale X-ray source setups.

Theoretical study of diffraction of evanescent X-rays and its relation to dispersion surface [4] has shown that diffraction in grazing incidence (GID) geometry is sensitive to the surface structure of the crystals. In particular, grazing incidence ensure a limited penetration depth, thus the beam mainly irradiates the surface and subsurface of the crystal. Near-surface horizontally propagating X-rays diffract from crystal planes perpendicular to the surface if the angle between the X-ray beam and the crystal planes satisfies Bragg's condition. This effect has been demonstrated experimentally [5].

However, the intensity of the diffracted beam is very low in GID, necessitating synchrotron-based experiments. Yet the specular-reflection intensity, at grazing incidence is much higher than that of diffraction. It has been shown in $[6,7]$ that specular-reflection intensity is modulated by the Bragg peak in GID. The intensity of the specular-reflection in the Bragg's condition for GID gives information about the structure of the subsurface layer; namely, thickness, amorphization and deformation (for example with use of static factor [8]).

However, the analysis discussed above is limited to the Bragg's condition. Yet information about the depth profile of the lateral lattice parameter and the relative lattice orientation is within a range far from GID Bragg's condition. In order to obtain the structural parameters of ultra-thin crystal subsurface layers, we here propose to extend the analysed angular range further away from the Bragg's condition. In numeric simulations we observed that a GID beam far from Bragg's condition also modulates a specular-reflection intensity.

In this chapter, we present a theoretical study of reflection intensity modulation involving the use of the matrix formalism of the dynamical diffraction theory [9]. Minor difference in structures of the bulk and the surface of the crystal significantly affect the GID intensity. The phase of the wave diffracted from the surface is slightly different from the phase of the wave diffracted from a bulk crystal substrate. Both these waves interfere, in a similar way to an acoustic beat, creating thickness oscillations with two distinguishable sets of frequencies on the rocking curves far from the Bragg's condition.

We have derived approximate expressions for the dispersion surface in order to understand how the parameters of the structure affect specular-reflection intensity modulation. In addition to thickness, deformation and amorphization, one can analyse the depth profiles of the lateral lattice parameter and the lattice orientation. Finally, a wider angular range allows us to increase the precision of estimation of these parameters compared to estimates in previous studies.

To study the feasibility of such measurement using low intensity sources, we have conducted numerical simulation of specular-reflection intensity while assuming 


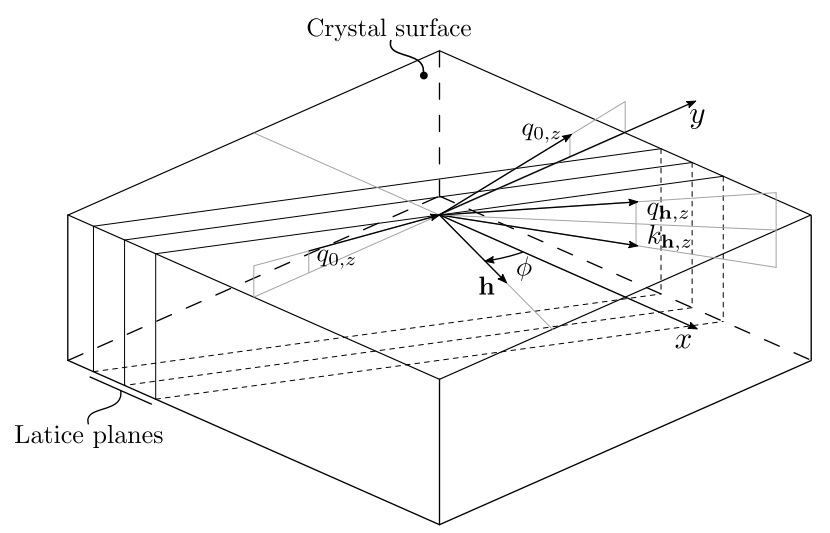

Figure 4.1: Geometry of the GID azimuthal rocking curves measurement.

typical parameters for lab-scale X-ray instrument. Based on the results of this simulation, we conclude that it is feasible to take such a measurement using the lab X-ray source.

\subsection{Theoretical background: Dynamical theory of diffraction}

In this section, we review the matrix formalism [10,11] of the dynamical diffraction theory [12] that we implement for the numerical simulations in Section 4.4. The problem of X-ray diffraction is approximated by the scalar wave equation [13]:

$$
\nabla^{2} E+[1+\chi(\mathbf{r})] q_{0}^{2} E=0
$$

where $E$ is the scalar amplitude of polarised electric wave, and $q_{0}=2 \pi / \lambda$ is the wave number of the wave in vacuum with wavelength $\lambda$. This equation was derived by assuming that diffraction is an elastic scattering process and the magnetic permittivity is equal to unity $\mu=1$. The crystal structure is represented with a dielectric susceptibility $\chi(\mathbf{r})$ as a function of coordinate $\mathbf{r}$.

In the GID geometry (see Fig. 4.1), one has to consider multiple scattering processes. Therefore, the dynamical theory of diffraction is used for GID. In that theory the solution of the wave equation is in the form of the Bloch wave [14]. The matrix formalism of the dynamical diffraction theory [10] considers two main scattering processes: reflection and diffraction. Within such a two-beam approximation, the wave field has the form:

$$
E(\mathbf{r})=E_{0} e^{i \mathbf{k}_{0} \cdot \mathbf{r}}+E_{\mathbf{h}} e^{i \mathbf{k}_{\mathbf{h}} \cdot \mathbf{r}},
$$

where $\mathbf{k}_{0}$ and $\mathbf{k}_{\mathbf{h}}=\mathbf{k}_{0}+\mathbf{h}$ are the wave vectors and $E_{0}$ and $E_{\mathbf{h}}$ are Fourier components of electric field corresponding to transmitted and diffracted waves respectively, 
and $|\mathbf{h}|=2 \pi / a$ is the reciprocal lattice vector, perpendicular to the lattice planes with spacing $a$. The dielectric susceptibility $\chi$ describes the optical properties of the medium [15]. Following the two-beam approximation, in a medium with a periodical local structure such as in a crystal, one can represent the susceptibility as a Fourier series:

$$
\chi(\mathbf{r})=\chi_{0}+\chi_{\mathbf{h}} e^{-i \mathbf{h} \cdot \mathbf{r}},
$$

where the $\chi_{0}$ component represents the amorphous properties of the medium, and $\chi_{\mathbf{h}}$ defines the local crystal structure. The reciprocal lattice vector $\mathbf{h}$ determines the family of lattice planes on which diffraction occurs.

Following the approximations in Eq. 4.2 and Eq. 4.3, one can derive the dispersion equation for s-polarized beam:

$$
\left(k_{0}^{2}-k^{2}\right)\left(k_{\mathbf{h}}^{2}-k^{2}\right)=q_{0}^{4} \chi_{\mathbf{h}} \chi_{-\mathbf{h}},
$$

where $k=\sqrt{1+\chi_{0}} q_{0}$ and is the wave number of the transmitted wave in the case of an amorphous medium. Wave numbers $k_{0}$ and $k_{\mathbf{h}}$ are associated with transmitted and diffracted waves in a crystal medium and differ from $k$ due to the dispersion in the crystal. An explicit analytical solution of the dispersion equation in GID geometry (close to the Laue-Bragg transition point) is given in [16]. For simplicity, we here consider the exact vector form of the dispersion equation and solve it numerically.

The tangential component of a wave vector is constant in all media as a result of translational invariance. The difference between the vertical components of the wave vectors is expressed as the aberration coefficient $\xi$; these two states can then be formulated as:

$$
\mathbf{k}_{0}=\mathbf{q}_{0}+q_{0} \xi \mathbf{e}_{z} ; \quad \mathbf{k}_{\mathbf{h}}=\mathbf{q}_{0}+q_{0} \xi \mathbf{e}_{z}+\mathbf{h},
$$

where $e_{z}$ is the unit vector along the $z$ axis normal to the surface of the crystal. Applying Eq. 4.5, the dispersion equation Eq. 4.4 becomes a polynomial equation of the fourth order with respect to $\xi$. Solutions of the dispersion equation Eq. 4.4 represent a geometrical surface - a dispersion surface. The Laue diffraction condition for vectors $\mathbf{k}_{0}$ and $\mathbf{k}_{\mathbf{h}}$ is satisfied at the intersection of the dispersion surfaces. Therefore, the shape of the dispersion surface describes the scattering process. We describe this in detail in Appendix 4.6.A.

To calculate amplitudes $E_{s}$ and $E_{\mathbf{h}}$ of specular-reflection and diffraction waves respectively for a layered sample, one needs to consider the continuity conditions for the electric field [15] in a stratified medium. A semi-infinite vacuum is separated from a semi-infinite crystal substrate by a stack of $N-1$ layers that have parallel interfaces. The vacuum medium has an index $i=0$ and the crystal is enumerated as $i=N$. Each $i$-th layer has a thickness $d_{i}$. Then applying continuity conditions to each interface one can derive:

$$
\mathbf{v}_{0}=\mathbf{M} \cdot \mathbf{v}_{N}
$$

where $\mathbf{v}_{0}=\left(1,0, E_{s}, E_{h}\right)^{T}$ and $\mathbf{v}_{N}=\left(D_{1}, D_{2}, 0,0\right)^{T}$ are vectors of values of the amplitudes in the vacuum and in the substrate respectively, $\mathbf{M}$ is the characteristic 
matrix of the sample:

$$
\mathbf{M}=\mathbf{M}_{0}^{-1} \mathbf{M}_{1} \mathbf{T}_{1} \mathbf{M}_{1}^{-1} \mathbf{M}_{2} \ldots \mathbf{T}_{N-1} \mathbf{M}_{N-1}^{-1} \mathbf{M}_{N}
$$

where $\mathbf{T}_{i}=\delta_{j k} \exp \left(i \kappa_{i}^{(j)} d_{i}\right)$ is a propagation matrix of the $i$-th layer, $\kappa_{i}=k_{0 i, z}^{(j)}$ is the $j$-th solution of the dispersion equation in the $i$-th layer and $\mathbf{M}_{i}$ is the characteristic matrix of the $i$-th layer. In the case of a mono-crystalline layer its characteristic matrix has the form:

$$
\mathbf{M}_{i}=\left[\begin{array}{cccc}
1 & 1 & 1 & 1 \\
\kappa_{i}^{(1)} & \kappa_{i}^{(2)} & \kappa_{i}^{(3)} & \kappa_{i}^{(4)} \\
c_{i}^{(1)} & c_{i}^{(2)} & c_{i}^{(3)} & c_{i}^{(4)} \\
u_{i}^{(1)} & u_{i}^{(2)} & u_{i}^{(3)} & u_{i}^{(4)}
\end{array}\right] ; \quad i \neq 0 .
$$

Here $c_{i}^{(j)}=\left(\left|\mathbf{q}_{\mathbf{0}}+q_{0} \xi_{i}^{(j)} \mathbf{e}_{z}\right|^{2}-k_{i}^{2}\right) / q_{0}^{2} \chi_{-\mathbf{h}, i}$ and $u_{i}^{(j)}=c_{i}^{(j)} \kappa_{i}^{(j)}$. In the special case of the vacuum medium, the characteristic matrix has the form:

$$
\mathbf{M}_{0}=\left[\begin{array}{cccc}
1 & 0 & 1 & 0 \\
q_{0, z} & 0 & -q_{0, z} & 0 \\
0 & 1 & 0 & 1 \\
0 & -q_{\mathbf{h}, z} & 0 & q_{\mathbf{h}, z}
\end{array}\right]
$$

Element $q_{\mathbf{h}, z}$ is the vertical component of the wave vector of the diffracted wave in the vacuum medium. Due to the translational invariance, the wave numbers of diffracted and incident wave are the same: $q_{0}=q_{\mathbf{h}}$. Therefore, one can derive:

$$
q_{\mathbf{h}, z}^{2}=q_{0}^{2}-\left(\mathbf{q}_{0}+\mathbf{h}\right)_{\|}^{2}
$$

Finally, solving Eq. 4.6 with respect to $E_{s}$ and $E_{h}$ allows calculation of the amplitudes of the specular-reflection and diffraction waves:

$$
E_{s}=\frac{M_{31} M_{22}-M_{32} M_{21}}{M_{11} M_{22}-M_{12} M_{21}} ; \quad E_{h}=\frac{M_{41} M_{22}-M_{42} M_{21}}{M_{11} M_{22}-M_{12} M_{21}} .
$$

\subsection{Geometry of the diffraction of evanescent $\mathrm{X}$ -} rays.

In general, the measurement of GID rocking curves is carried out through an azimuthal rotation of the sample (rotation around the normal to the surface). We denote the angle between vector $\mathbf{h}$ and the $x y$ plane as $\psi$. Fig. 4.1 shows a typical measurement geometry for GID, where for clarity of the drawing the lattice planes are chosen to be perfectly perpendicular to the crystal surface $(\psi=0)$, although the equations in Section 5.2 allow any orientation of the lattice planes. For $\psi=0$ the reciprocal lattice vector $\mathbf{h}$ lies in the surface plane. The coordinate system is chosen such that the tangential projection of the incident wave vector lies in the plane $y z$ and the $x y$ plane is parallel to the surface. Thereby, the orientation of the crystal planes is described by the position of the vector $\mathbf{h}$, i.e. by azimuthal angle $\phi$. 
The Bragg's condition in this geometry can be formulated as $\mathbf{q}_{0} \cdot \mathbf{h}=-q_{0} h \sin \theta_{\mathrm{B}}$, where $\theta_{\mathrm{B}}$ is the Bragg angle. Since lattice planes are chosen to be perpendicular to the surface, the diffracted wave propagates towards the bottom of the crystal: $R e k_{\mathbf{h} z}<0$. If the grazing angle of incidence is slightly above the critical angle $\theta_{0} \in\left(\theta_{c}, 2 \theta_{c}\right)$ then the diffracted wave $\mathbf{k}_{\mathbf{h}}$ also propagates at an angle nearly parallel to the surface and reflects from the surface $[4,17]$.

The nature of that evanescent diffracted wave with wave vector $\mathbf{q}_{\mathbf{h}}$ is counter intuitive. On the one hand, that wave has a diffraction nature, but on the other hand it clearly does not satisfy the Laue diffraction condition $\mathbf{q}_{h}-\mathbf{q}_{0} \neq \mathbf{h}$, since $q_{\mathbf{h} z}>0$. However, Eq. 4.10 holds, and that yields the interesting result that a small change of the azimuthal angle $\phi$ will lead to a much larger change in the diffraction exit angle $\theta_{\mathbf{h}}$. The ratio can be two to three orders of magnitude, depending on wavelength and lattice spacing.

The fact that $\theta_{h}$ changes during azimuthal rotation is not the only consequence of Eq. 4.10. One can notice that in the case of $q_{0}^{2}<\left(\mathbf{q}_{0}+\mathbf{h}\right)_{\|}^{2}$, Eq. 4.10 has no solution in real numbers. In that case, by analogy with the effect of total external reflection, the evanescent diffracted wave is extinct. The point $q_{0}^{2}=\left(\mathbf{q}_{0}+\mathbf{h}\right)_{\|}^{2}$ is termed as the Laue point. By analogy with the situation of a critical angle, the Laue point is the azimuthal angle position below which a GID beam cannot exit the crystal.

\subsection{Diffraction of an evanescent wave from a dis- torted subsurface layer}

In this section, we discuss predictions of the dynamical theory for the diffraction of a GID wave on a distorted crystal subsurface. The model sample is a silicon single crystal incorporating a thin "distorted" single crystalline Si layer with a lattice mismatch on top. Layer and substrate consist of the same material, and the lateral lattice mismatch of $0.1 \%$ is not sufficient to change the optical density, hence there is no optical contrast between the subsurface layer and the bulk of the single crystal. In this example, the thickness of the layer is $d=9 \mathrm{~nm}$.

Consider the ideal case in which the Laue point is close to the Bragg's condition. In that case, the crystal planes are perpendicular to the surface of the sample. As described in Section 4.3, in this case the Bragg's condition is satisfied for the diffracted wave propagating towards the bottom of the crystal but due to the small angle of propagation that beam partially reflects and exits the crystal through the surface. That process is due to the diffraction that occurs in the lateral direction, and the phase rapidly changes in the vertical direction due to constraints on the wave vector in a vacuum medium, Eq. 4.10. That makes this scattering process sensitive to the lateral crystal structure with respect to its vertical displacement from the surface of the sample.

Although the model of the sample have not any optical contrast, there are actually strong thickness oscillations (see Fig. 4.2a). Three details are relevant about these oscillations. First, the frequency of the oscillations varies with the azimuthal angle $\phi$. Second, the thickness oscillations have a duplet structure: an 

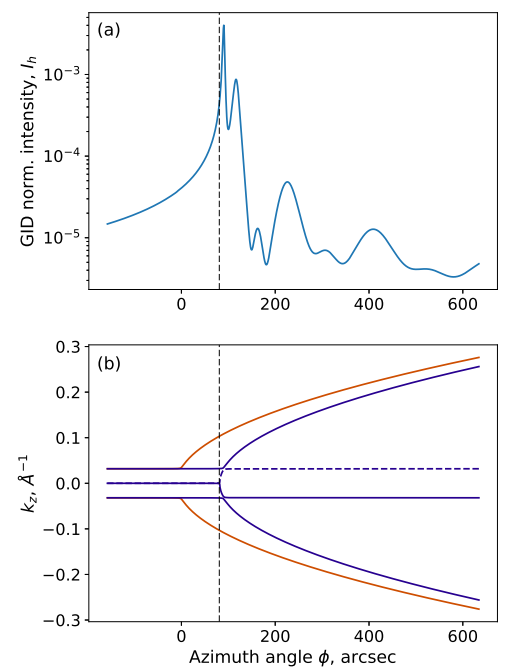

Figure 4.2: Simulation of a GID azimuthal rocking curve. Model: Incident angle $\theta_{0}=0.5^{\circ}$, wavelength $\lambda=0.154 \mathrm{~nm}$. Si mono-crystal, surface orientation (001), scattering crystal planes series $\{220\}$, no miscut $\psi=0^{\circ}$; with $9 \mathrm{~nm}$ distorted crystalline Si layer with lateral lattice constant difference $1-a_{\text {Crystal }} / a_{\text {layer }}=0.1 \%$. (a) GID azimuthal rocking curve. (b) Dispersion surface cross-section. Orange curves - dispersion in the substrate, blue curves - dispersion in the subsurface layer, blue dashed curve - neglected root of dispersion equation.

oscillation consists of two peaks. Third, there are no oscillations for azimuthal angles bellow the Laue point. The Laue point for the layer is shown in Fig. 4.2 as a vertical black dashed line.

To address these features, we refer to the cross-section of the dispersion surface. The curves in Fig. 4.2b show the dependency of the vertical component of the wave vectors with respect to the azimuthal position. Essentially, these wave vectors describe the propagation angles of the waves in the sample. There are two branches of the dispersion surface: the orange curves are due to the waves in the substrate and the blue curves are due to the waves in the distorted layer.

During azimuthal rotation of the sample, the angles of propagation of transmitted and reflected wave are constant. Therefore, transmitted and reflected waves are represented by horizontal lines. Note that the dispersion curves of the transmitted and reflected waves in the layer and substrate coincide. That is due the absence of any optical contrast. The wave reflected on the interface between layer and substrate (black dashed line) is negligible due to the small angle of incidence and the absence of optical contrast. For this reason, that wave was neglected in the three-roots approach (see Appendix 4.6.B).

The dispersion curves of the diffracted waves diverge in the range above the Laue point, because the propagation angle of the diffracted wave changes with azimuthal angle, as discussed in Section 4.3. That is the reason why the frequency of the thickness oscillations shifts with azimuthal angle. The difference between orange and blue branches (see Fig. 4.2b) yields the duplet shape of the oscillations 

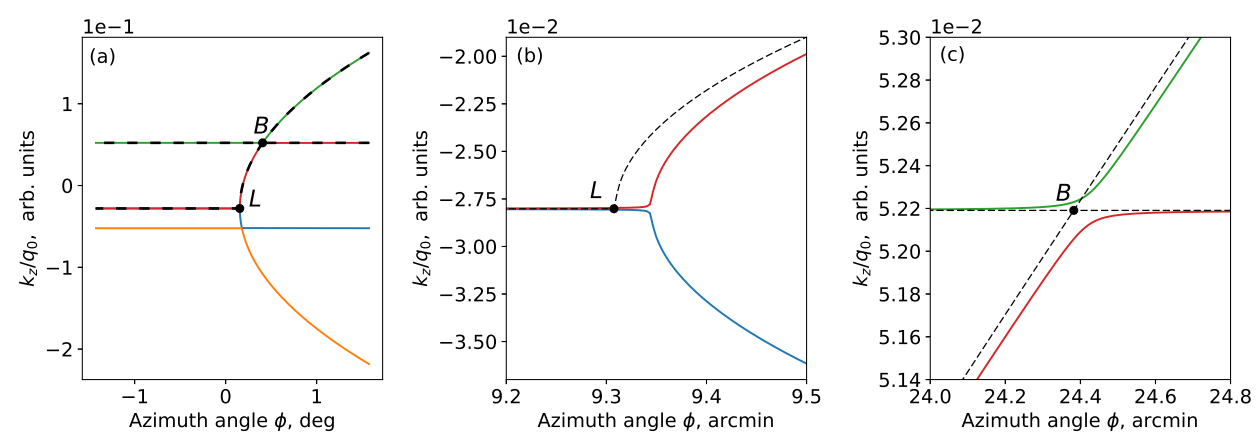

Figure 4.3: Exact (solid lines) and approximated (dashed lines) solutions of the dispersion equation. Model: Incident angle $\theta_{0}=3^{\circ}$, wavelength $\lambda=0.154 \mathrm{~nm}$. Single Si crystal, surface orientation (001), scattering crystal planes series $\{220\}$, miscut angle $\psi=2^{\circ}$; (a) Large scale (b) Near the Laue point (c) Close to Bragg condition.

in Fig. 4.2a. The physical explanation for this phenomenon is the fact that there are two evanescent waves propagating through the layer representing diffraction in both the layer and in the substrate.

Now consider approximated analytic solutions of the dispersion equation. To derive these we will approximate the dispersion equation by a continuity condition, expressed as Eq. 4.10. Following that approach, solutions for the specular waves simply converge to translational invariance:

$$
\frac{k_{0, z}}{q_{0}}= \pm\left(\chi_{0}+\sin ^{2} \theta_{0}\right)^{1 / 2} .
$$

Assuming that $\left|\mathbf{q}_{\mathbf{h}}\right| \approx\left|\mathbf{k}_{\mathbf{h}}\right|$ for diffracted waves we derive:

$$
k_{\mathbf{h}, z}^{2} \propto \sin ^{2} \theta_{0}-\gamma_{B}^{2} \cos ^{2} \psi-2 \gamma_{B} \eta
$$

where $\eta=\cos \psi \cos \theta_{0} \sin \phi$ and $\gamma_{B}=2 \sin \theta_{B}=\lambda / a$ for lattice constant $a$. The approximation $\left|\mathbf{q}_{\mathbf{h}}\right| \approx\left|\mathbf{k}_{\mathbf{h}}\right|$ is due to the fact that in an in-plane geometry the asymmetry of the diffraction is significantly higher than the refraction. Yet the refraction can be taken into account by a factor $1+\chi_{0}$ into the left-hand term of Eq. 4.10. The Laue point then satisfies equality:

$$
\sin ^{2} \theta_{0}-\gamma_{B}^{2} \cos ^{2} \psi=2 \gamma_{B} \eta
$$

Now, consider the displacement related to the miscut angle $\psi$ :

$$
\frac{k_{\mathbf{h}, z}}{q_{0}} \approx \pm\left(\sin ^{2} \theta_{0}-\gamma_{B}^{2} \cos ^{2} \psi-2 \gamma_{B} \eta\right)^{1 / 2}-\gamma_{B} \sin \psi
$$

Thus, Eq. 4.15 and Eq. 4.12 are approximated solutions of the dispersion equation. The comparison of the approximated and exact solution is shown in Fig. 4.3. Therefore the diffraction, is the result of coupled oscillations of these two evanescent waves. 

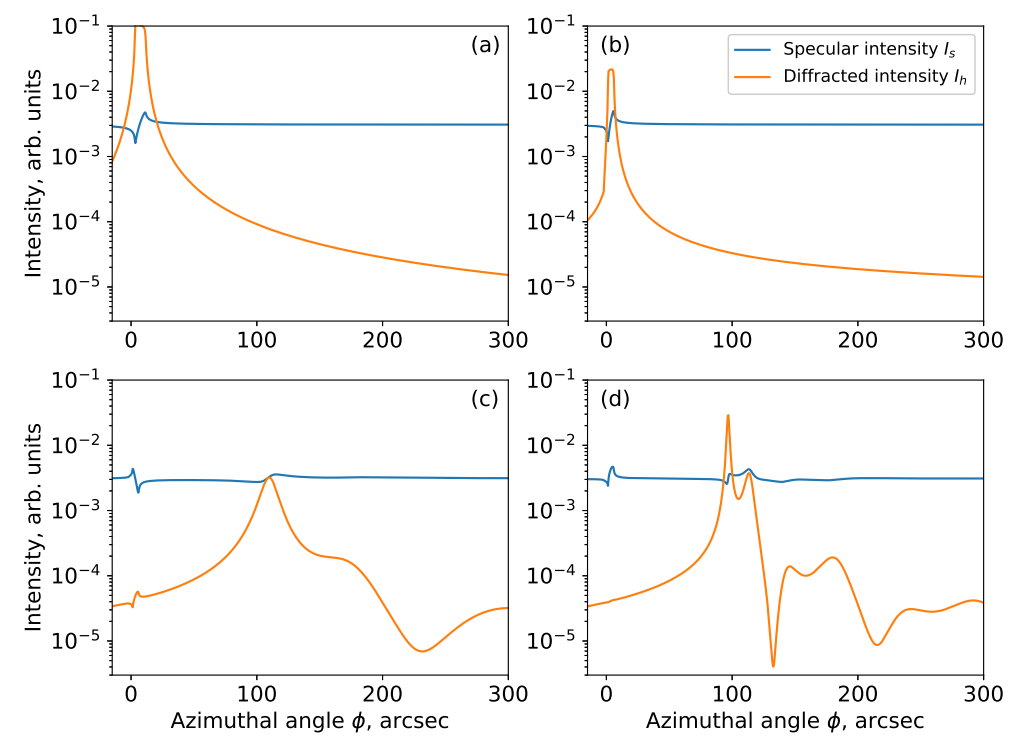

Figure 4.4: Simulations of the specular reflection intensity and GID azimuthal rocking curve. Model: Si mono-crystal with various thicknesses of distorted layer: (a) $d=0 \mathrm{~nm}$ (b) $d=2 \mathrm{~nm}$ (c) $d=5 \mathrm{~nm}$ and (d) $d=12 \mathrm{~nm}$. Incident angle $\theta_{0}=0.5^{\circ}$, wavelength $\lambda=0.154 \mathrm{~nm}$. Single Si crystal, surface orientation (001), scattering crystal planes series $\{220\}$, miscut angle $\psi=2^{\circ}$.

This approximate solution can be used for easy analysis of the frequencies of the thickness oscillations, omitting complex numerical simulations. Finally, below the Laue point the dispersion curves converge to zero and remain constant. Within that range, diffracted waves in the layer are extinct and there are no interference, and thus no oscillations.

Now, by an analogy with the study of amorphous layers with specular-reflection intensity [18], let us consider how diffraction of the evanescent wave in the distorted layer affects the specular-reflection. To observe modulation of the specular-reflection intensity by GID, a small miscut of $\psi=1^{\circ}$ is considered in the model of the sample. Fig. 4.4 shows a simulation of the specular-reflection and evanescent diffraction for various thicknesses of the top layer, as a function of the azimuthal angle around substrate Bragg peak.

For the simulations shown in Fig. 4.4a there is no top layer present, and one can observe that the intensity of the specular-reflection intensity is modulated by the diffraction from the substrate Bragg peak alone. With a thickness $d=2 \mathrm{~nm}$ of the top layer (see Fig. 4.4b), the diffracted intensity of the Bragg peak from the substrate decreases drastically and one can note how thickness oscillations are appearing. In Fig. 4.4c, one can see the shape of the Bragg peak from a distorted crystal layer at a thickness of $d=5 \mathrm{~nm}$. At this thickness, both diffraction from the subsurface and diffraction from the substrate simultaneously modulate the specularreflection. At a $12 \mathrm{~nm}$ layer, there is no peak of diffraction from the substrate but there is still a modulation to the specular-reflection. That can be explained by the 


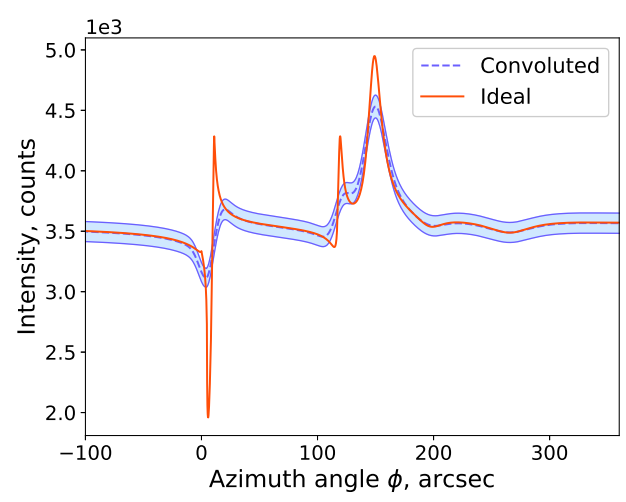

Figure 4.5: Specular reflection intensity. Comparison between ideal curve and measurement simulation. Instrument model parameters: Ge $\{220\}$ monochromator, wavelength $\left(\mathrm{CoK}_{\alpha}-\right.$ line) $\lambda=0.18 \mathrm{~nm}$, photon energy dispersion $\delta E=1.1 \mathrm{eV}$, incidence angle $\theta_{0}=0.7^{\circ}$, angular divergence in azimuthal direction $\delta \phi=15$ arcsec, direct beam intensity $I=10^{4}$ c.p.s., measurement time 85 min. Sample model: Single Si crystal, surface orientation (001), scattering crystal planes series $\{220\}$, miscut angle $\psi=1^{\circ}$, with distorted $d=7 \mathrm{~nm}$ thick layer.

fact that there is no diffracted wave propagating towards the surface, but there is still a transmitted diffracted wave in the substrate propagating toward the bottom of the crystal.

Notice that for the case of a thick subsurface layer shown in Fig. 4.4d, there is almost no Bragg peak from the substrate visible in the GID rocking curve. However, modulation of the specular-reflection intensity by the Bragg peak from the substrate is still observed. This can be used in an experiment as a reference point in order to estimate the difference of lattice constants between the distorted layer and the substrate. The approximated solution Eq. 4.15 of the dispersion equation depends on the lattice constant $\gamma_{B}=\lambda / a$ and the miscut angle $\psi$. The form of this equation implies that it would be possible to distinguish the influence of the lattice constant and the miscut angle on the frequencies and the shape of oscillations during the experiment.

Finally, in order to check the feasibility of specular-reflection intensity modulation, we conducted numerical simulation. The results of this numerical simulation are shown in Fig. 4.5.

Here we consider the typical parameters of lab X-ray instrument. For higher resolutions, compared to conventional CuK-line X-ray tube, we consider an X-ray tube with a lower photon energy: CoK-line. We have considered the Ge $\{220\}$ monochromator. The spectral width of the Ge $\{220\}$ peak for the CoK-line is $\delta E=$ $1.03 \mathrm{eV}$. For the simulation, we consider the worst parameters for which features of the ideal curve are still resolvable: photon energy dispersion $\delta E=1.1 \mathrm{eV}$, angular divergence in azimuthal direction $\delta \phi=15$ arcsec, direct beam intensity considered to be low $I=10^{4}$ c.p.s. due to a strong collimation of the beam. Measurement uncertainties were estimated considering that measurement took 90 minutes. Thus we conclude the feasibility of specular-reflection intensity modulation measurement on lab-scale X-ray diffractometer 
As a logical next step, experimental evaluation of these new predictions of the dynamical theory would be necessary. In particular, the simplicity of the specular geometry and the high intensity of the reflected beam provide an opportunity to implement the technique on a relatively (compared to synchrotron) low power lab based X-ray diffractometer, as distinct from a synchrotron-based system.

\subsection{Conclusions}

Implementation of the matrix formalism of the dynamical X-ray diffraction theory allowed us to describe theoretically a novel scattering process for single crystals that have defects in the crystal structure of the subsurface layers, that were introduced by the interaction with the atmosphere. GID waves induced in the subsurface interface and on the surface yield strongly asymmetric azimuthal curves. That asymmetry allowed us to estimate the difference between lattice constants of the subsurface and crystal substrate, the thickness of the distorted subsurface structure, the difference in miscut between subsurface and substrate, and the optical contrast. Based on obtained approximate solutions of dispersion equation we conclude that these parameters are uncorrelated. It was also shown by means simulations that GID also modulates the specular-reflection which potentially allows one to take measurements using lab-based instruments, making the technique widely accessible to researchers.

\subsection{Appendix}

\subsection{A Numerical simulation of the dispersion surface}

The roots $\xi$ of Eq. 4.4 are aberration coefficients. They determine the deviations from the vertical components of the wave vectors. Tangential components are known due to translational invariance. So, for a given set of $\xi$ calculated for every possible incident wave vector $\mathbf{q}_{0}$ and using Eq. 4.5, one can calculate the dispersion surface. The physical interpretation of the roots of the dispersion equation is that each of them is assigned to a component of a Bloch as wave expressed in Eq. 4.2. Thus, one can distinguish the role of a particular component in the scattering process.

For simplicity, we first consider the trivial case of conventional symmetric X-ray diffraction. The cross-section of the dispersion surface for that case is shown in Fig. 4.6. The axes of Fig. 4.6 represent the vertical and the tangential components of the wave vectors. The surface of the crystal is shown schematically in Fig. 4.6a as a solid black line at the center. In the case of symmetric X-ray diffraction, the lattice planes are parallel to the surface (see Fig. 4.6a). It is similar to an Ewald construction, considering two spheres of $k=\sqrt{1+\chi_{0}} q_{0}$ radius. The distance between the centers of the spheres is the length of the reciprocal lattice vector $\mathbf{h}$. The sphere that has it center in $\left(k_{z}, k_{\|}\right)=(0,0)$ (blue circle) represents the set of $\mathbf{k}_{0}$ vectors while the other sphere (orange circle) represents the set of $\mathbf{k}_{\mathbf{h}}$ vectors. The point of intersection corresponds to the Bragg angle.

Due to dispersion, absolute values of incident and diffracted waves are not restricted to being the same. Indeed, close to the Bragg angle one can see deviations 


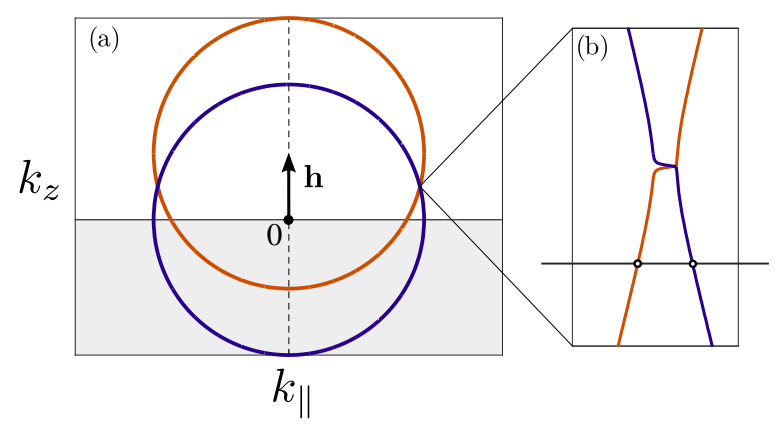

Figure 4.6: The dispersion surface cross-section of symmetrical diffraction in co-planar geometry. Scattering planes Si $\{111\}$ lattice planes family. Surface orientation (111). a) Large scale. b) Small scale near Bragg's condition.

of the dispersion surface from a spherical shape; see Fig. 4.6b. This is qualitatively explained by the formation of a standing wave under the Bragg's conditions due to the coherence between the incident and the scattered waves. Fig. 4.6b shows that in the case of symmetrical diffraction there are two roots of the dispersion equation involved.

In the case in which the lattice planes are not parallel to the surface of the crystal, the process of X-ray diffraction is asymmetric. The angle of the reflected beam and the exit angle of the diffracted beam are no longer equal. The shape of the dispersion surface is different in that case, see Fig. 4.7. It can be observed that the Ewald sphere is rotated with reciprocal lattice vector $\mathbf{h}$ at an angle $\psi$. In this case, part of the blue sphere (corresponding to $\mathbf{k}_{0}$ ) to the right from the dotted dash line in Fig. 4.6b is not covered above with the orange sphere (corresponding to $\mathbf{k}_{\mathbf{h}}$ ). This means that an incident beam unnecessarily spawns a diffracted beam exiting the crystal. The dotted vertical dash line represents the Laue point. At that point, the configuration of the scattering process is changing. The diffracted beam propagates towards the bottom of the crystal instead of exiting through the surface. The effect of the Bragg - Laue transition on in-plane diffraction curves is considered in Section 4.3.

The shape of the dispersion surface close to the Bragg condition shows that three roots of the dispersion equation are involved in the diffraction (see Fig. 4.7b). The fourth root is far on the other side of the Ewald sphere. In other words, the wave represented by this root is not coherent to the other waves; therefore, its contribution to the diffraction is negligible. We assume that in any geometry at least one wave is not coherent to the others. Following that assumption, we modify the matrix formalism to accommodate only three roots of the dispersion equation, as discussed in Appendix 4.6.B.

\subsection{B Comparison of simulations that use three roots and four roots matrices}

The propagation matrix $\mathbf{T}$ may become poorly conditioned due to exponents in its elements. Reducing the number of elements in the matrix $\mathbf{T}$ can significantly 


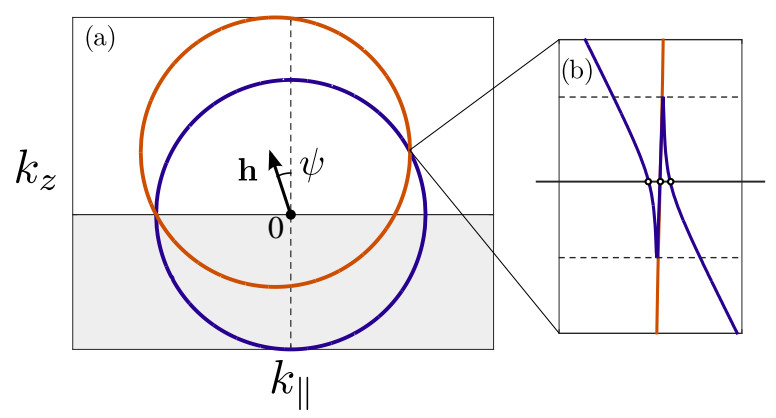

Figure 4.7: The dispersion surface cross-section of asymmetrical diffraction in co-planar geometry. Scattering planes Si $\{111\}$ lattice planes family. Surface orientation (111). a) Large scale. b) Small scale near Bragg's condition.

improve the calculation stability. Therefore, following our assumption (see Appendix 4.6.A) that only three roots of the dispersion Eq. 4.4 are relevant, one can reduce the matrix, neglecting the root of the incoherent wave:

$$
\mathbf{M}_{i}=\left[\begin{array}{ccc}
1 & 1 & 1 \\
\kappa_{i}^{(1)} & \kappa_{i}^{(2)} & \kappa_{i}^{(3)} \\
c_{i}^{(1)} & c_{i}^{(2)} & c_{i}^{(3)} \\
u_{i}^{(1)} & u_{i}^{(2)} & u_{i}^{(3)}
\end{array}\right] ; \quad i \neq 0 .
$$

For given atomic coordinates in a unit cell and atomic scattering factors for each atom in that unit cell, one can calculate the components of the susceptibility:

$$
\chi_{\mathbf{g}}=-\frac{r_{\mathrm{el}} \lambda^{2}}{\pi V} \sum_{s} f_{s} e^{-i \mathbf{g} \cdot \mathbf{r}_{s}} ; \quad \mathbf{g}=0, \mathbf{h},-\mathbf{h},
$$

where $V$ is the unit cell volume, $r_{\mathrm{el}}-$ the classical electron radius, $r_{s}-$ the coordinates of atom in a unit cell and $f_{s}$ - the atomic scattering factor [19]. Summation is performed over all atoms $s$ in a unit cell of the lattice.

Next, the dispersion equation Eq. 4.4 is solved and each root is associated with an amplitude in an array from one to four. The first root should correspond to a transmitted wave, the second to a diffracted wave towards the bottom of a crystal, the third to a specular-reflection wave and the fourth to a diffracted wave towards the surface. Next, roots of the incoherent wave are neglected and then via Eq. 4.7 and Eq. 4.11 amplitudes $E_{s}$ and $E_{h}$ are calculated. Finally, the intensities are calculated:

$$
I_{s}=\left|E_{s}\right|^{2} ; \quad I_{h}=\left|E_{h}\right|^{2}\left|\frac{q_{h, z}}{q_{0, z}}\right| .
$$

We tested these approaches with three and four roots for simulation of symmetric diffraction and reflection. The discrepancies concerned the levels of machine epsilon. For the GID geometry, discrepancies were highest at a level of $\delta I \approx 10^{-5}$. However, such discrepancies are irrelevant for practical applications. Thus, threeroot approximation was used for all numerical examples described in this chapter. 


\section{References}

[1] P. Ngabonziza, R. Heimbuch, N. de Jong, R. A. Klaassen, M. P. Stehno, M. Snelder, A. Solmaz, S. V. Ramankutty, E. Frantzeskakis, E. van Heumen, G. Koster, M. S. Golden, H. J. W. Zandvliet, and A. Brinkman, In situ spectroscopy of intrinsic $\mathrm{Bi}_{2} \mathrm{Te}_{3}$ topological insulator thin films and impact of extrinsic defects, Phys. Rev. B 92, 035405 (2015).

[2] B. A. Aronzon, M. V. Kovalchuk, E. M. Pashaev, M. A. Chuev, V. V. Kvardakov, I. A. Subbotin, V. V. Rylkov, M. A. Pankov, I. A. Likhachev, B. N. Zvonkov, Y. A. Danilov, O. V. Vihrova, A. V. Lashkul, and R. Laiho, Structural and transport properties of $G a A s / \delta-M n / G a A s / I n_{x} G a_{1-x} A s / G a A s$ quantum wells, Journal of Physics: Condensed Matter 20, 145207 (2008).

[3] I. K. Robinson, Crystal truncation rods and surface roughness, Phys. Rev. B 33, 3830 (1986).

[4] A. M. Afanas'ev and M. K. Melkonyan, X-ray diffraction under specular reflection conditions. Ideal crystals, Acta Crystallographica Section A 39, 207 (1983).

[5] P. L. Cowan, S. Brennan, T. Jach, M. J. Bedzyk, and G. Materlik, Observations of the diffraction of evanescent $x$ rays at a crystal surface, Phys. Rev. Lett. 57, 2399 (1986).

[6] V. A. Bushuev and A. P. Oreshko, Specular X-ray reflection from a crystal coated with an amorphous film under the conditions for strongly asymmetric noncoplanar diffraction, Physics of the Solid State 43, 941 (2001).

[7] V. A. Bushuev, R. M. Imamov, É. K. Mukhamedzhanov, and A. P. Oreshko, Specular reflection of X-rays under the conditions of grazing diffraction in a crystal with an amorphous surface layer, Crystallography Reports 46, 909 (2001).

[8] A. Afanasev, M. Kovalchuk, E. Kovev, and V. Kohn, X-ray diffraction in a perfect crystal with disturbed surface layer, physica status solidi (a) 42, 415 (1977).

[9] S. Stepanov and R. Kohler, A dynamical theory of extremely asymmetric Xray diffraction taking account of normal lattice strain, Journal of Physics D: Applied Physics 27, 1922 (1994).

[10] S. A. Stepanov, E. A. Kondrashkina, R. Köhler, D. V. Novikov, G. Materlik, and S. M. Durbin, Dynamical X-ray diffraction of multilayers and superlattices: Recursion matrix extension to grazing angles, Phys. Rev. B 57, 4829 (1998).

[11] A. Caticha, Diffraction of X-rays at the far tails of the Bragg peaks, Phys. Rev. B 47, 76 (1993).

[12] Z. G. Pinsker, Dynamical Scattering of X-rays in Crystals, Vol. 3 (SpringerVerlag Berlin, 1978). 
[13] U. Pietsch, V. Holý, and T. Baumbach, High-Resolution X-Ray Scattering From Thin Films to Lateral Nanostructures (Springer New York, 2004).

[14] V. Hol and P. F. Fewster, Dynamical diffraction in layered systems - a quest for the final formula, Journal of Physics D: Applied Physics 36, A5 (2003).

[15] M. Born and E. Wolf, Principles of optics: electromagnetic theory of propagation, interference and diffraction of light (Elsevier, 2013).

[16] V. M. Kaganer, V. L. Indenbom, M. Vrána, and B. Chalupa, Laue-to-Bragg transition in extremely asymmetric dynamic neutron diffraction, physica status solidi (a) 71, 371 (1982).

[17] P. L. Cowan, Diffraction of evanescent X-rays: Results from a dynamical theory, Phys. Rev. B 32, 5437 (1985).

[18] V. A. Bushuev and A. P. Oreshko, X-ray specular reflection under conditions of extremely asymmetric noncoplanar diffraction from a bicrystal, Crystallography Reports 48, 180 (2003).

[19] B. L. Henke, E. M. Gullikson, and J. C. Davis, X-ray interactions: photoabsorption, scattering, transmission, and reflection at $E=50-30000 \mathrm{eV}, Z=1-92$, Atomic data and nuclear data tables 54, 181 (1993). 




\subsection{Introduction}

Achievements in the field of science and technology related to the manufacture of nanoscale devices are associated with the systematic decrease of the characteristic sizes of the structure of such devices. Decrease in characteristic size leads to the fact that the performance of some nanoscale devices is affected by minor variations in its structure geometry, elemental composition and atomic distribution of different elements inside the structure. Examples of such structures can be found in microelectronic industry [1,2]. Understanding and improving the performance of such devices therefore requires the use of nanometrology techniques that are capable to reconstruct geometry of the structure, the elemental composition and the three dimensional atomic concentration distributions of different elements. Such element selective analysis can be done by measuring X-ray fluorescence excited by the grazing-incidence radiation (GIXRF). When an X-ray standing wave (XSW) is excited in an object, the GIXRF intensity will be determined by the XSW amplitude at the location of the fluorescent atoms. By varying the angle of incidence and/or incident photon energy, the location of XSW field nodes and anti-nodes can be varied in depth. Consequently, the emission of GIXRF induced by the electric field depends on the incident angle and the incident photon energy, as well as on the spatial distribution of the fluorescent atoms.

Measurement procedures and data analysis for one dimensional depth distributions of fluorescent atoms have been well developed [3] and implemented for the study of epitaxial layers [4], multilayers [5] and Langmuir-Blodgett films [6], among others. However, properties of some nanoscale devices like light-trapping structures in solar cells [4], field emitter arrays [7] and nanorods [8] depend on their complex 2D or 3D architecture. The depth atomic distribution profile of such structures can still be analyzed in the framework of conventional 1D XSW method with use of the effective layer approximation [9]. In this approximation, the atomic concentration distribution is averaged along lateral directions. Such approach does not take into account diffraction on the lateral structure of the sample and therefore is applicable only in case of randomly distributed objects along latteral direction of the structure. Inherently, the information about lateral distribution is lost within the effective layer approach, and the effective atomic concentration profile can never fully explain the properties of such $2 \mathrm{D}$ or $3 \mathrm{D}$ devices.

In recent work [10], the sensitivity of GIXRF to the lateral distribution of atomic concentration in a 3D structure of periodically arranged nano-columns has been experimentally demonstrated. To achieve such sensitivity, a new experimental scheme has been employed. Measurements were done under different grazing incidence and azimuthal orientation angles, unlike conventional XSW experiments, where measurements are done solely with respect to the grazing angle of incidence. The optical matrix method [11] used for the analysis in conventional XSW [5] does not allow analysis of the lateral distribution of atomic concentration.

The problem of GIXRF data analysis for complex structures has been addressed in [12]. There the 2D structure of a lamellar grating has been analyzed. The experimentally measured GIXRF curves were analyzed by solving the Maxwell's equations by means of the finite-element method (FEM) [13]. However applicability of FEM 
is limited due to its high demand in computational effort. FEM's computational effort increases with the increase of the incident photon energy, the size and the dimensionality of the structure. For instance, FEM simulations for the experiment published in [10] are practically irrealizable. This problem has been recognized by the scientific community [12] and a new computational scheme will have to be developed to allow the simulation of GIXRF under conditions in which use of the FEM approach is not practical.

In this study we derive semi-analytic equations based on the dynamical diffraction theory for the calculation of GIXRF intensity. We derive the solution of Sherman equation $[14,15]$ for the GIXRF intensity induced by XSW in the 3D periodic structure in linearalgebraic form. In order to test the new computational scheme, we perform numerical simulations for the 2D structure of lamellar grating and qualitatively compare them with the results of FEM simulations and measurements published in [12]. The semi-analytical nature of the derived equations allowed us to strongly reduce the computational effort, and to perform analysis of GIXRF from a $3 \mathrm{D}$ nanostructured surface for the first time (experimental data previously published in [10]).

\subsection{Theory}

In this section we consider a theory which will be used further for the analysis of GIXRF experimental data measured from 2D and 3D structures. The problem of calculation of GIXRF intensity is divided on two parts: calculation of the near field excited in the structure by the incident beam, and calculation of the corresponding fluorescence intensity using Sherman [15] equation. In Sections 5.2.1 - 5.2.3 we consider the theoretical background of the dynamical diffraction theory in many beam approximation (MBDDT) [16]. In Section 5.2.4 we consider a solution to the problem of numerical stability which occurs in the numerical simulations. In Section 5.2.5 we derive the solution of the Shermann equation in linear-algebraic form, which will further allow us to calculate GIXRF intensity of 2D and 3D structures.

\subsubsection{Many beam dynamical diffraction theory}

Experimental geometry used in [10, 12] for the GIXRF measurments is shown in Fig. 5.1a. An X-ray beam impinges onto a sample surface under grazing incident angle $\alpha_{i}$ and azimuthal angle $\phi$. Excited GIXRF intensity is measured using an energy-dispersive silicon drift detector $D$. In order to calculate the fluorescent intensity from the sample, the near field needs to be calculated. The problem of near field $(\mathrm{NF})$ calculation is formulated by the Helmholtz equation:

$$
\left(\Delta+k_{0}^{2}\right) E(\mathbf{r})=-k_{0}^{2} \chi(\mathbf{r}) E(\mathbf{r})
$$

Here, for simplicity we consider the Helmholtz equation in a scalar approximation, $E(\mathbf{r})$ is the electric field, the sample structure is represented by the dielectric susceptibility function $\chi(\mathbf{r})$ and $k_{0}=2 \pi / \lambda$ is the wave number of the incident beam with the wavelength $\lambda$. The Helmholtz equation can be solved using the finite element method (FEM), kinematical diffraction theory or dynamical diffraction 


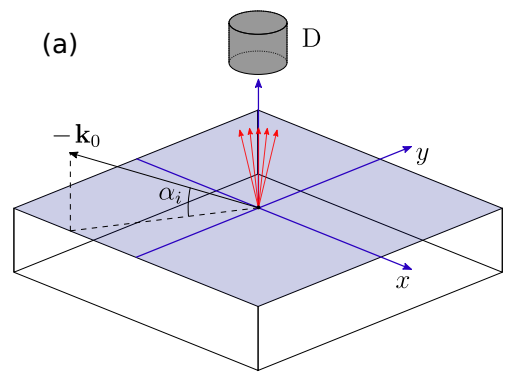

(b)

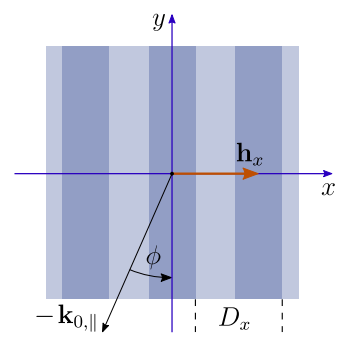

(c)

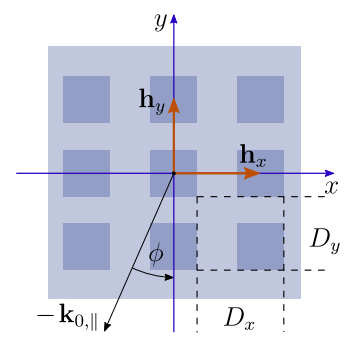

Figure 5.1: a) Experimental geometry of GIXRF; $D$ - energy-dispersive silicon drift detector, $\alpha_{i}$ - angle of incidence, $\mathbf{k}_{0}$ - wave vector of the incident beam. b) Sketch of a typical 2D periodic structure, with azimuthal rotation angle $\phi$. c) Sketch of a typical 3D periodic structure.

theory. With FEM being computationally challenging, and kinematical theory is not sufficiently precise under grazing incidence conditions [16], we further consider dynamical diffraction theory. Furthermore, to take into account the lateral structure of the sample one needs to consider dynamical diffraction theory in many beam approximation (MBDDT).

In the dynamical diffraction theory, Eq. 5.1 is solved assuming that NF is represented as a Bloch wave:

$$
E(\mathbf{r})=\sum_{\mathbf{h}} E_{\mathbf{h}}(z) \exp \left(i \mathbf{k}_{\mathbf{h} \|} \cdot \mathbf{r}\right),
$$

and the structure is represented as the Fourier series:

$$
\chi(\mathbf{r})=\sum_{\mathbf{h}} \chi_{\mathbf{h}} \exp (i \mathbf{h} \cdot \mathbf{r}),
$$

where $\mathbf{h}=\mathbf{h}_{x}+\mathbf{h}_{y}$, is the reciprocal space vector with components

$$
\mathbf{h}_{x, y}=\frac{2 \pi n_{x, y}}{D_{x, y}} \mathbf{e}_{x, y},
$$

with order of diffraction $n_{x, y}$ and $D_{x, y}$ the periods along $x$ or $y$ directions respectively. The parallel component of the wave vector of the $h$-th diffraction order $\mathbf{k}_{\mathbf{h} \|}=\mathbf{k}_{0 \|}+\mathbf{h}$ is translationally invariant along the $z$ direction; i.e. $\mathbf{k}_{\mathbf{h} \|}$ is constant across all medias in the multilayer system for given $h$, while the vertical component is generally different in each medium and defined with the spherical dispersion equation:

$$
q_{\mathbf{h} z}^{2}=k_{0}^{2}\left(1+\chi_{0}\right)-k_{\mathbf{h} \|}^{2} .
$$

This equation is derived assuming that diffraction scattering is an elastic process: $k_{\mathbf{h}}=\left(1+\chi_{0}\right) k_{0}$ and assuming translational invariance of $\mathbf{k}_{\mathbf{h} \|}$ mentioned above. Finally, substituting Eq. 5.2, Eq. 5.3 and Eq. 5.4 in Eq. 5.1 result in a system of inhomogeneous linear ordinary differential equations (ODE) of second order:

$$
q_{\mathbf{h} z}^{2} E_{\mathbf{h}}(z)+\frac{\mathrm{d}^{2}}{\mathrm{~d} z^{2}} E_{\mathbf{h}}(z)+k_{0}^{2} \sum_{\mathbf{g} \neq \mathbf{h}} E_{\mathbf{g}}(z) \chi_{\mathbf{g}-\mathbf{h}}=0 .
$$


The general solution of such a system of ODE is a linear combination of particular solutions of corresponding homogeneous ODEs, where the $n$-th particular solution has the form of a standing wave with amplitudes $T_{n}$ and $R_{n}$.

Thus, the h-th solution of Eq. 5.5 has the form:

$$
E_{\mathbf{h}}(z)=\sum_{n}\left[T_{n} \exp \left(-i k_{n z} z\right)+R_{n} \exp \left(i k_{n z} z\right)\right] E_{h n}
$$

with linear combination coefficients $E_{h n}$. Therefore, the distribution of the NF is defined with Eq. 5.2 and Eq. 5.6. Thus, the problem of NF calculation is reduced to finding $k_{n, z}, E_{h n}, T_{n}$ and $R_{n}$.

\subsubsection{Characteristic equation}

In this section we discuss the calculation of $k_{n, z}$ and $E_{h n}$. Variable $k_{n, z}$ has a physical meaning as the vertical component of the wavevector (see Eq. 5.6). It defines the phase of the standing wave in the structured layer. One can assume that $k_{n, z}$ is defined with spherical dispersion $k_{z}=q_{z}$, however under that assumption Eq. 5.5 has no solutions. Therefore, values of $k_{n, z}$ deviate from spherical dispersion. To calculate the precise value of $k_{n, z}$ in the structured layer one can substitute Eq. 5.6 in Eq. 5.5. The result is represented as the eigenvalues-eigenvectors problem:

$$
\left(\mathbf{A}-k_{z n}^{2} \mathbf{I}\right) \mathbf{E}_{n}=\mathbf{0}
$$

whre $k_{z n}^{2}$ is an eigenvalue of matrix $\mathbf{A}$ and $\mathbf{E}_{n}$ is an eigenvector composed of the coefficients $E_{h n}$ from Eq. 5.6: $\mathbf{E}_{n}=\left(\ldots E_{-1, n}, E_{0, n}, E_{1, n} \ldots\right)^{T} ; \mathbf{A}$ is of the form:

$$
\mathbf{A}=k_{0}^{2} \mathbf{C}-\mathbf{X}
$$

Matrix $\mathbf{C}$ is the Toeplitz circulant matrix:

$$
\mathbf{C}=\left[\begin{array}{lllll}
\ddots & & & & \\
& \chi_{0} & \chi_{-1} & \chi_{-2} & \\
& \chi_{1} & \chi_{0} & \chi_{-1} & \\
& \chi_{2} & \chi_{1} & \chi_{0} & \\
& & & & \ddots
\end{array}\right]
$$

and $\mathbf{X}$ is the diagonal matrix with diagonal $\left(\cdots-k_{-1, \|}^{2},-k_{0, \|}^{2},-k_{1, \|}^{2} \ldots\right)$. Circulant matrices have a remarkable property: with increasing circulant matrix size, its eigenvalues asymptotically approach the exact values for an infinite matrix [17]. Therefore, one can use a finite amount of Fourier components in Eq. 5.3 to approximate the exact solution of Eq. 5.5. Consider a set of $2 N+1$ Fourier components $\left\{\chi_{-N}, \chi_{-N+1}, \ldots, \chi_{0}, \ldots \chi_{N-1}, \chi_{N}\right\}$. These Fourier components constitutes a circulant matrix $\mathbf{C}$ of a size $\mathbf{C} \in \mathbb{C}^{M \times M}$, where $M=N+1$. Solving the characteristic Eq. 5.7 will give $M$ eigenvalue-eigenvector pairs. 


\subsubsection{Boundary conditions}

In this section we calculate amplitudes $T_{n}$ and $R_{n}$. Consider a sample as a stratified medium, consisting of layers. $T_{n}$ and $R_{n}$ are calculated in each layer using continuity conditions of the electric field and its first derivative. The continuity conditions [18] for the $j$-th and $(j+1)$-th pair of layers can be written in a matrix form:

$$
\mathbf{P}^{(j)}\left[\begin{array}{c}
\mathbf{T}^{(j)} \\
\mathbf{R}^{(j)}
\end{array}\right]=\mathbf{P}^{(j+1)} \mathbf{Q}^{(j+1)}\left[\begin{array}{c}
\mathbf{T}^{(j+1)} \\
\mathbf{R}^{(j+1)}
\end{array}\right] .
$$

Here $\mathbf{T}$ and $\mathbf{R}$ are vectors composed of amplitudes $T_{n}$ and $R_{n}$ :

$$
T=\left(T_{-N / 2}, T_{-N / 2+1}, \ldots, T_{0}, \ldots T_{N / 2-1}, T_{N / 2}\right)^{T} .
$$

Eq. 5.10 links amplitudes $\mathbf{T}^{(j)}, \mathbf{R}^{(j)}$ at the interface between $(j-1)$-th and $j$-th layer, and amplitudes $\mathbf{T}^{(j+1)}, \mathbf{R}^{(j+1)}$ at the interface between $j$-th and $(j+1)$-th layer, Matrix $\mathbf{P}$ is the refraction matrix. For a structured layer it has a form:

$$
\mathbf{P}=\left[\begin{array}{cc}
\mathbf{E} & \mathbf{E} \\
-\mathbf{E k}_{z} & \mathbf{E k}_{z}
\end{array}\right]
$$

and for a homogeneous layer:

$$
\mathbf{P}=\left[\begin{array}{cc}
\mathbf{I} & \mathbf{I} \\
-\mathbf{k}_{z} & \mathbf{k}_{z}
\end{array}\right]
$$

Here, the matrix $\mathbf{E}$ is composed of columns of eigenvectors and matrix $\mathbf{k}_{z}$ is a diagonal matrix filled with $k_{z, n}$. Refraction matrix $\mathbf{P}$ is a $2 \times 2$ block matrix, thus $\mathbf{P} \in \mathbb{C}^{2 M \times 2 M}$. Finally $\mathbf{Q}$ is the propagation matrix:

$$
\mathbf{Q}=\left[\begin{array}{cc}
\mathbf{Q}^{+} & \mathbf{0} \\
\mathbf{0} & \mathbf{Q}^{-}
\end{array}\right]
$$

where $\mathbf{Q}^{ \pm}$are diagonal matrices with corresponding diagonals:

$$
\left(\ldots e^{\mp i k_{-h, z} d_{j}}, e^{\mp i k_{0, z} d_{j}}, e^{\mp i k_{h, z} d_{j}} \ldots\right),
$$

where $d_{j}$ is the thickness of $j$-th layer. Although these equations can be used to calculate $\mathbf{T}_{j}$ and $\mathbf{R}_{i}$, solving Eq. 5.10 might be problematic due to the poorly conditioned transmission matrix in case of sufficiently large thickness of the sample and/or in case of sufficiently high number of Fourier components used in the calculation.

\subsubsection{Numerical stability}

The problem of numerical stability in the matrix formalism of dynamical diffraction theory was considered in [19]. There the problem of numerical stability has been solved for the dynamical diffraction theory in two-beam approximation (only $\chi_{-1}$, $\chi_{0}$ and $\chi_{1}$ has been taken into account). It has been solved by dividing matrices in $2 \times 2$ block matrices and solving Eq. 5.10 separately for each block matrix by using 


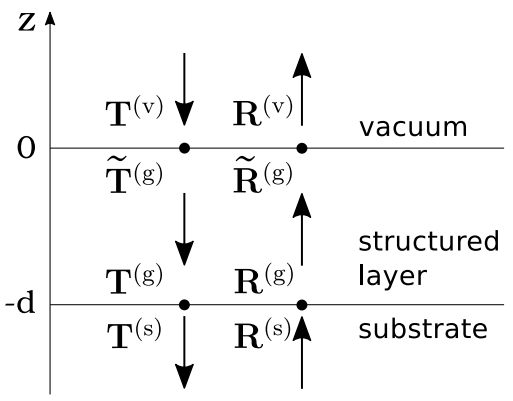

Figure 5.2: Sketch of the three layer model. Arrows schematically depicts the direction of propagation of the plane waves. Amplitudes of the plane waves are assembled into $\mathbf{T}$ and $\mathbf{R}$ vectors. $\widetilde{\mathbf{T}}$ and $\widetilde{\mathbf{R}}$ are amplitudes defined at the upper interface of the layer. $\mathbf{T}$ and $\mathbf{R}$ are defined at the bottom interface of the layer.

recurrent formula. Although the recurrent matrix equations in [19] were derived for the two-beam case, they are generally applicable to the many-beam case. For brevity, we present these equations explicitly written for a three-layer system (see Fig. 5.2), which is relevant to the experimental data we will considered further. The continuity conditions for such a three-layer structure (vacuum - structured layer substrate) are represented by the system of linear equations:

$$
\begin{gathered}
{\left[\begin{array}{l}
\mathbf{T}^{(\mathrm{v})} \\
\mathbf{R}^{(\mathrm{v})}
\end{array}\right]=\left(\mathbf{P}^{(\mathrm{v})}\right)^{-1} \mathbf{P}^{(\mathrm{g})} \mathbf{Q}^{(\mathrm{g})}\left[\begin{array}{l}
\mathbf{T}^{(\mathrm{g})} \\
\mathbf{R}^{(\mathrm{g})}
\end{array}\right] ;} \\
{\left[\begin{array}{l}
\mathbf{T}^{(\mathrm{g})} \\
\mathbf{R}^{(\mathrm{g})}
\end{array}\right]=\left(\mathbf{P}^{(\mathrm{g})}\right)^{-1} \mathbf{P}^{(\mathrm{s})}\left[\begin{array}{l}
\mathbf{T}^{(\mathrm{s})} \\
\mathbf{R}^{(\mathrm{s})}
\end{array}\right] .}
\end{gathered}
$$

One can rewrite that system as follows:

$$
\left[\begin{array}{l}
\mathbf{T}^{(\mathrm{g})} \\
\mathbf{R}^{(\mathrm{v})}
\end{array}\right]=\mathbf{M}^{\mathrm{vg}}\left[\begin{array}{l}
\mathbf{T}^{(\mathrm{v})} \\
\mathbf{R}^{(\mathrm{g})}
\end{array}\right] ; \quad\left[\begin{array}{l}
\mathbf{T}^{(\mathrm{s})} \\
\mathbf{R}^{(\mathrm{g})}
\end{array}\right]=\mathbf{M}^{\mathrm{gs}}\left[\begin{array}{l}
\mathbf{T}^{(\mathrm{g})} \\
\mathbf{R}^{(\mathrm{s})}
\end{array}\right]
$$

Here matrix $\mathbf{M}$ has the form of a block matrix:

$$
\mathbf{M}=\left[\begin{array}{cc}
\mathbf{Q}^{-} \mathbf{V}_{11}^{-1} & -\mathbf{Q}^{-} \mathbf{V}_{11}^{-1} \mathbf{V}_{12} \mathbf{Q}^{-} \\
\mathbf{V}_{21} \mathbf{V}_{11}^{-1} & \mathbf{V}_{22} \mathbf{Q}^{-}-\mathbf{V}_{21} \mathbf{V}_{11}^{-1} \mathbf{V}_{12} \mathbf{Q}^{-}
\end{array}\right]
$$

where $\mathbf{V}_{i j}$ is a matrix element of $2 \times 2$ block matrix $\mathbf{V}^{(\mathrm{vg}, \mathrm{gs})}=\left(\mathbf{P}^{(\mathrm{v}, \mathrm{g})}\right)^{-1} \mathbf{P}^{(\mathrm{g}, \mathrm{s})}$ Note that this equation does not include $\mathbf{Q}^{+}$which elements are growing exponentially with respect to the thickness of the structured layer. Hence this matrix is numerically stable. Amplitudes $\mathbf{T}^{(\mathrm{v})}$ represent the incident beam, therefore

$$
\mathbf{T}^{(\mathrm{v})}=(\ldots 0,1,0 \ldots)^{T} .
$$

Additionally, for a sufficiently thick substrate we can assume

$$
\mathbf{R}^{(\mathrm{s})}=(\ldots 0,0,0 \ldots)^{T} .
$$


Taking into account these considerations, we derive equations for amplitudes in the structured layer:

$$
\mathbf{R}^{(\mathrm{g})}=\left(\mathbf{I}-\mathbf{M}_{21}^{(\mathrm{gs})} \mathbf{M}_{12}^{(\mathrm{vg})}\right)^{-1} \mathbf{M}_{21}^{(\mathrm{gs})} \mathbf{M}_{11}^{(\mathrm{vg})} \mathbf{T}^{(\mathrm{v})}
$$

and:

$$
\mathbf{T}^{(\mathrm{g})}=\left(\mathbf{I}-\mathbf{M}_{12}^{(\mathrm{vg})} \mathbf{M}_{21}^{(\mathrm{gs})}\right)^{-1} \mathbf{M}_{11}^{(\mathrm{vg})} \mathbf{T}^{(\mathrm{v})}
$$

These amplitudes are calculated at the interface between the structured layer and the substrate (see Fig. 5.2). One can calculate amplitudes at the vacuum-structured layer interface using:

$$
\left[\begin{array}{c}
\widetilde{\mathbf{T}}^{(\mathrm{g})} \\
\widetilde{\mathbf{R}}^{(\mathrm{g})}
\end{array}\right]=\left(\mathbf{P}^{(\mathrm{g})}\right)^{-1} \mathbf{P}^{(\mathrm{v})}\left[\begin{array}{c}
\mathbf{T}^{(\mathrm{v})} \\
\mathbf{R}^{(\mathrm{v})}
\end{array}\right]
$$

Finally, we need to rewrite Eq. 5.6:

$$
E_{\mathbf{h}}(z)=\sum_{n}\left[\widetilde{T}_{n} \exp \left(-i k_{n z} z\right)+R_{n} \exp \left(i k_{n z}[z+d]\right)\right] E_{h n}
$$

Here, both exponents decrease with respect to the depth, providing numerical stability.

\subsubsection{X-ray fluorescence intensity}

The fluorescence intensity $Y$ can be calculated with use of Sherman equation [14], adapted for GIXRF [15]:

$$
Y \propto G\left(\alpha_{i}\right) \iiint|E(\mathbf{r})|^{2} p(\mathbf{r}) \exp (-\mu \rho z) d \mathbf{r}
$$

where $p(\mathbf{r})$ is the numeric density of fluorescent atoms which describes its distribution in the structure, and $G\left(\alpha_{i}\right)$ is the geometrical factor [20-22]. The integral is taken over the area of thr elementary cell. The exponential term $\exp (-\mu \rho z)$ in Eq. 5.23 takes into account the absorption of fluorescent photons. Here, $\mu$ is the absorption coefficient and $\rho$ is the effective density of the absorbing media. The integral in Eq. 5.23 can be separated as follows (further, for brevity we do not explicitly write the multiplicative term $\left.G\left(\alpha_{i}\right)\right)$ :

$$
\begin{aligned}
Y \propto \sum_{\mathbf{g}, \mathbf{h}} \iint p\left(\mathbf{r}_{\|}\right) \exp \left(i\left[\mathbf{k}_{\mathbf{g} \|}-\mathbf{k}_{\mathbf{h} \|}^{*}\right] \cdot \mathbf{r}\right) d x d y \times \\
\left.\quad \times \sum_{m, n} \int\left[T_{m} \exp \left(-i k_{m z} z\right)+R_{m} \exp \left(i k_{m z} z\right]\right)\right] \times \\
\times\left[T_{n}^{*} \exp \left(i k_{n z}^{*} z\right)+R_{n}^{*} \exp \left(-i k_{n z}^{*} d\right)\right] E_{g m} E_{h n}^{*} \exp (-\mu \rho z) d z
\end{aligned}
$$

Such integral separation imposes a restriction on numeric density function: it must not be dependent on $z$ coordinate $p(\mathbf{r}) \equiv p\left(\mathbf{r}_{\|}\right)$; i.e. Eq. 5.24 can only be used in 
cases when fluorescent atoms are distributed homogeneously along the $z$ direction. Distribution in the $x y$-plane can be arbitrary. In case of an inhomogeneous vertical distribution, one can discretize the structure along the $z$ direction as a stack of sublayers and calculate Eq. 5.24 for each sublayer.

Eq. 5.23 was rewritten in the form of Eq. 5.24, so it can be conveniently represented in a linear algebraic language. The fluorescence intensity can be expressed as the sum of matrix elements $Y \propto \sum_{g, h} F_{g h}$ of the matrix:

$$
\mathbf{F}=\mathbf{\Phi} \circ\left(\mathbf{E} \mathbf{\Psi} \mathbf{E}^{*}\right) \text {. }
$$

Here $\circ$ represent element-wise (Hadamard) multiplication. Elements of matrix $\mathbf{\Phi}$ have the form:

$$
\Phi_{h g} \equiv \int_{-D_{x} / 2}^{D_{x} / 2} d x \int_{-D_{y} / 2}^{D_{y} / 2} d y p\left(\mathbf{r}_{\|}\right) \exp \left(i\left[\mathbf{k}_{\mathbf{g} \|}-\mathbf{k}_{\mathbf{h} \|}^{*}\right] \cdot \mathbf{r}\right),
$$

and elements of matrix $\boldsymbol{\Psi}$ have the form:

$$
\begin{aligned}
\Psi_{m n} \equiv T_{m} T_{n}^{*} U\left(-k_{z m}+k_{z n}^{*}\right)+T_{m} R_{n}^{*} U\left(-k_{z m}-k_{z n}^{*}\right)+ \\
+R_{m} T_{n}^{*} U\left(k_{z m}+k_{z n}^{*}\right)+R_{m} R_{n}^{*} U\left(k_{z m}-k_{z n}^{*}\right),
\end{aligned}
$$

where

$$
U(q) \equiv \int_{-d}^{0} \exp (i q z) \exp (-\mu \rho z) d z .
$$

The $\boldsymbol{\Phi}$ matrix takes into account the distribution of fluorescent atoms and the electric field distribution in lateral direction and the $\boldsymbol{\Psi}$ matrix takes into account photon absorption and the electric field distribution in vertical direction. Eq. 5.25 allows to calculate the integral in Eq. 5.23 analytically, which is much more computationally efficient compared to the numerical integration.

Thus, the fluorescence intensity calculation includes the following steps:

- Calculate the Fourier components in Eq. 5.3 of the elementary cell of the structure and create a Toeplitz matrix Eq. 5.9 using Fourier components.

- For each pair of angles $\left(\alpha_{i}, \phi\right)$ calculate the eigenvalues $k_{z n}^{2}$ and the corresponding matrix of eigenvectors $\mathbf{E}$ using the dispersion equation (Eq. 5.7).

- Calculate amplitudes $\mathbf{R}^{(\mathrm{g})}$ using Eq. 5.19 and $\widetilde{\mathbf{T}}^{(\mathrm{g})}$ using Eq. 5.21.

- Finally, calculate GIXRF intensity using Eq. 5.25.

\subsection{Numerical simulations}

In this section we compare the theory derived in Section 5.2.5 with experimental results. The purpose of this comparison is to determine whether the theory is appropriate for further implementation of the dynamical diffraction theory as a tool for the reconstruction of atomic concentration profiles. We will use a simple model taking into account one structured surface layer and the homogeneous substrate (see Fig. 5.2). 


\subsubsection{D structure: $\mathrm{Si}_{3} \mathrm{~N}_{4}$ lamelar grating}

Here, we consider a $2 \mathrm{D}$ lamelar $\mathrm{Si}_{3} \mathrm{~N}_{4}$ grating prepared using electron beam lithography. The original study with experimental data and numerical simulation of GIXRF intensity by means of FEM has been published by Soltwisch et. al. [12]. The grating has a nominal period of $D_{x}=100 \mathrm{~nm}$, the thickness of the structured layer is $d=90 \mathrm{~nm}$ and the line width is $40 \mathrm{~nm}$.

The GIXRF measurements were carried out at the plane-grating monochromator (PGM) beamline [23] for undulator radiation at the PTB laboratory [24] of the BESSY II synchrotron radiation facility. A monochromatic excitation with a photon energy of $520 \mathrm{eV}$. was used. GIXRF intensity curves were measured for the $\mathrm{N}-\mathrm{K} \alpha$ line under various incidence angle $\alpha_{i}$ and azimuthal sample orientation angle $\phi$ (see Fig. 5.1b); $\phi=0^{\circ}$ corresponds to the conical orientation [25] of the sample grating. The recorded spectra were deconvoluted using detector response functions in order to isolate the fluorescence signal from $\mathrm{N}-\mathrm{K} \alpha$ from other spectral contributions. Further corrections, to take into account the detection efficiency and the geometrical factor (effective solid angle) were applied.

Best-fit simulations based on theory described in Section 5.2, and experimental GIXRF curves are shown in Fig. 5.3 for various azimuthal orientation angles $\phi$. For the simulation we use a simple box model, in which the grating lines are treated as an
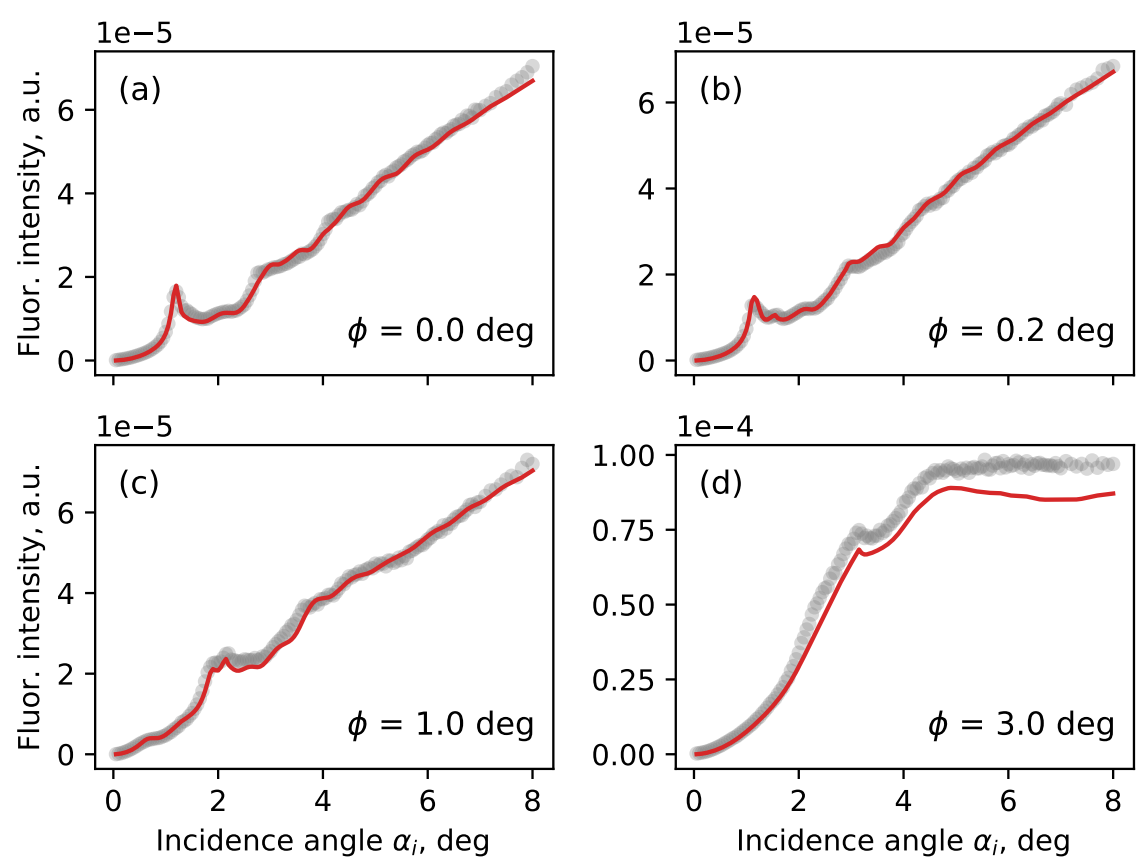

Figure 5.3: $\mathrm{N}-\mathrm{K} \alpha$ GIXRF intensity curves, measured for various azimuthal orientation angles (a) $\phi=0^{\circ}$ - conical, (b) $\phi=0.2^{\circ}$, (c) $\phi=1^{\circ}$ and (d) $\phi=3^{\circ}$. Red lines - numerical simulation, gray markers - experimental data. 

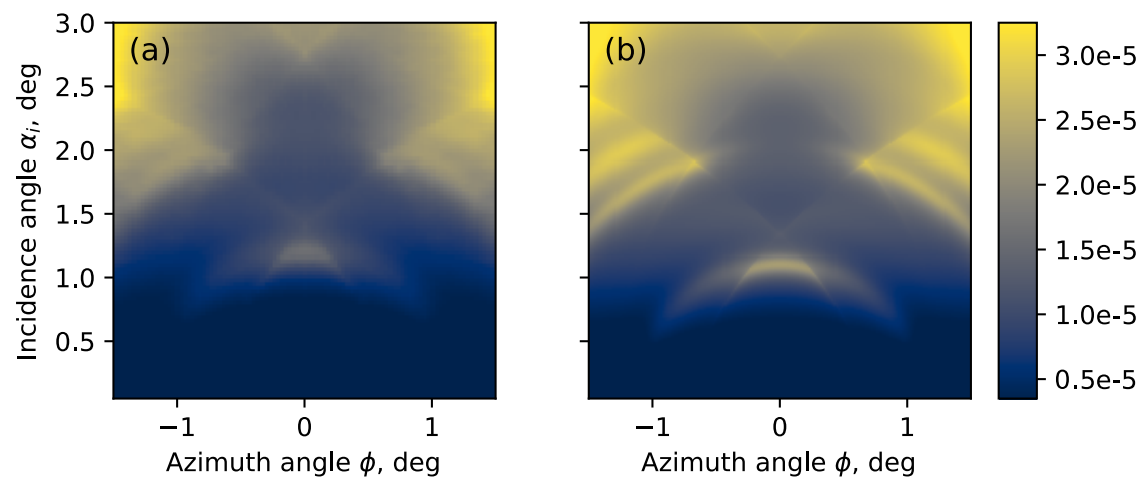

Figure 5.4: (2D XSW) Comparison between the experimental GIXRF map (a) of the $\mathrm{Si}_{3} \mathrm{~N}_{4}$ lamelar grating measured with the incidence photon energy $E=520 \mathrm{Ev}$ and the simulated GIXRF map (b) based on a best fit model.

array of boxes on top of the substrate. Thus, the medium is divided into three areas: the vacuum, the structured layer in which the boxes are located, and the substrate. Within box model, sidewalls of the grating line are considered to be parallel while the actual grating has a sidewall tilt angle. Based on the reconstruction in [12], this angle is not greater than $\beta=4^{\circ}$. In terms of the model it means that the Fourier transform in Eq. 5.3 is changing along $z$ axis. To compensate for that in the simulations, within one layer model averaged Fourier components have been used, i.e. $\left\langle\chi_{h}\right\rangle=\chi_{h} \exp \left(-h^{2} \sigma^{2} / 2\right)$, with $\sigma$ defined as half the projection of the sidewall on the $x$ axis: $\sigma \equiv d \arctan (\beta) / 2$. Best-fit line width (defined as the half-height width) is $D_{l}=43 \mathrm{~nm}$ and the best-fit sidewall tilt angle $\beta=5^{\circ}$.

Another feature of the actual sample that must be considered in the simulations is the effect of surface oxidation. It affects the actual structure such that the concentration of fluorescent $\mathrm{N}$ atoms at the top part is strongly reduced. In the one layer model, oxidation of the surface can be effectively incorporated by changing the integration limits in Eq. 5.28, such that the integration in Eq. 5.28 is taken only over a range where fluorescent atoms are present. The best agreement with experimental data was obtained with an effective oxide layer thickness of $d_{0}=1.5 \mathrm{~nm}$. Note that this value only describes the effect of an oxide layer in terms of absence of fluorescent $\mathrm{N}$ atoms, ignoring the gradual change in stoichiometry throughout the oxide layer. It also neglects the change of optical properties of the structure due to oxidation. Best-fit parameter of the grating height, including effective oxide layer, is $d=92 \mathrm{~nm}$. Average density of the grating line is $\rho=2.8 \mathrm{~g} / \mathrm{cm}^{3}$.

Best-fit model and experimental data are qualitatively in good agreement. Qualitative agreement is also apparent on GIXRF intensity $\left(\alpha_{i}, \phi\right)$-maps shown in Fig. 5.4. A full set of 48 experimental GIXRF curves taken along different azimuthal angles $\phi$ (from $0^{\circ}$ untill $2^{\circ}$ ) was interpolated on a $\left(\alpha_{i}, \phi\right)$ grid (see Fig. 5.4a). The theoretical GIXRF map was calculated on the same $\left(\alpha_{i}, \phi\right)$ grid using best fit parameters from the data presented in Fig. 5.4. 


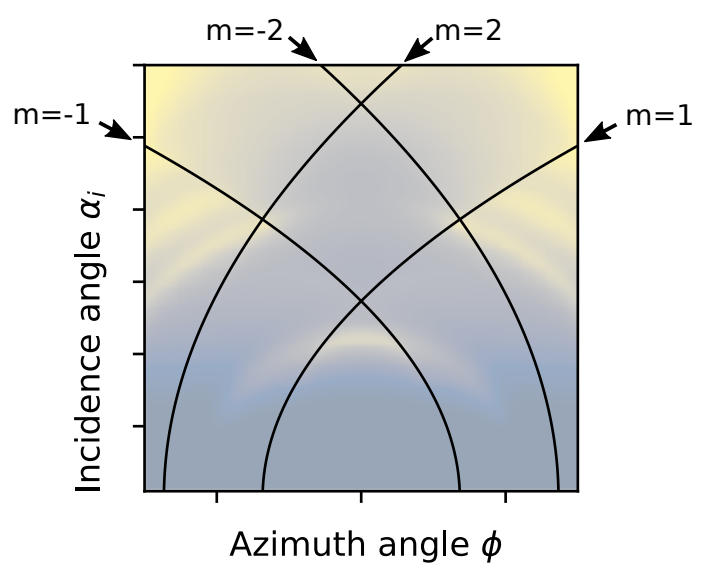

Figure 5.5: Resonant lines in GIXRF map for $\mathrm{Si}_{3} \mathrm{~N}_{4}$ grating structure, caused by interference between reflected beam and $\mathrm{m}$-th order of diffraction.

One can note a distinctive feature on the GIXRF map - resonant lines also known in literature as Yoneda lines [26]. Resonant lines are visible on experimental data in Fig. 5.4a and they are reproduced in the numerical simulations (see Fig. 5.4b). As a visual aid to notice these lines one can refer to the sketch in Fig. 5.5. In Fig. 5.5 the position of the resonant lines is marked with black contour lines. We assume that these lines are due to the interference between the reflected beam (0-th order of diffraction) and a diffracted beam ( $m$-th order of diffraction). Therefore, the resonant lines must satisfy the Laue condition, which for this geometry can be formulated as $k_{x}^{2}+k_{z}^{2}=\left(k_{x}+h\right)^{2}$. This formula geometrically corresponds to the Ewald sphere. For convenience we rewrite this equation in terms of incidence and azimuth angles:

$$
\sin \phi=\frac{\sin ^{2} \alpha_{i}-\gamma^{2}}{2 \gamma \cos \alpha_{i}},
$$

where $\gamma=\lambda m / D_{x}$. The contour lines in Fig. 5.5 were calculated using this equation. Note that the resonant lines depend only on the lateral period of the structure $\gamma$ (see Eq. 5.29, no other structural parameters play a role. Due to their explicit dependence on only the period, such lines might be used in the analysis of experimental data as a reference, to determine the lateral period of the structure, without needing a full structure reconstruction through model simulations.

\subsubsection{D structure: Cr nano-columns}

In this section we consider a 3D nano-columnar structure of $\mathrm{Cr}$, manufactured using electron beam lithography [27] on top of a $\mathrm{SiO}_{2}$ substrate. The structure of the sample is a regular square grid of box-shaped columns (see Fig. 5.1c) on a substrate, with $300 \mathrm{~nm} \times 300 \mathrm{~nm}$ lateral box dimensions and a $D_{x}=D_{y}=1 \mu \mathrm{m}$ grid. The nominal height of the nano-columns is $d=25 \mathrm{~nm}$. 

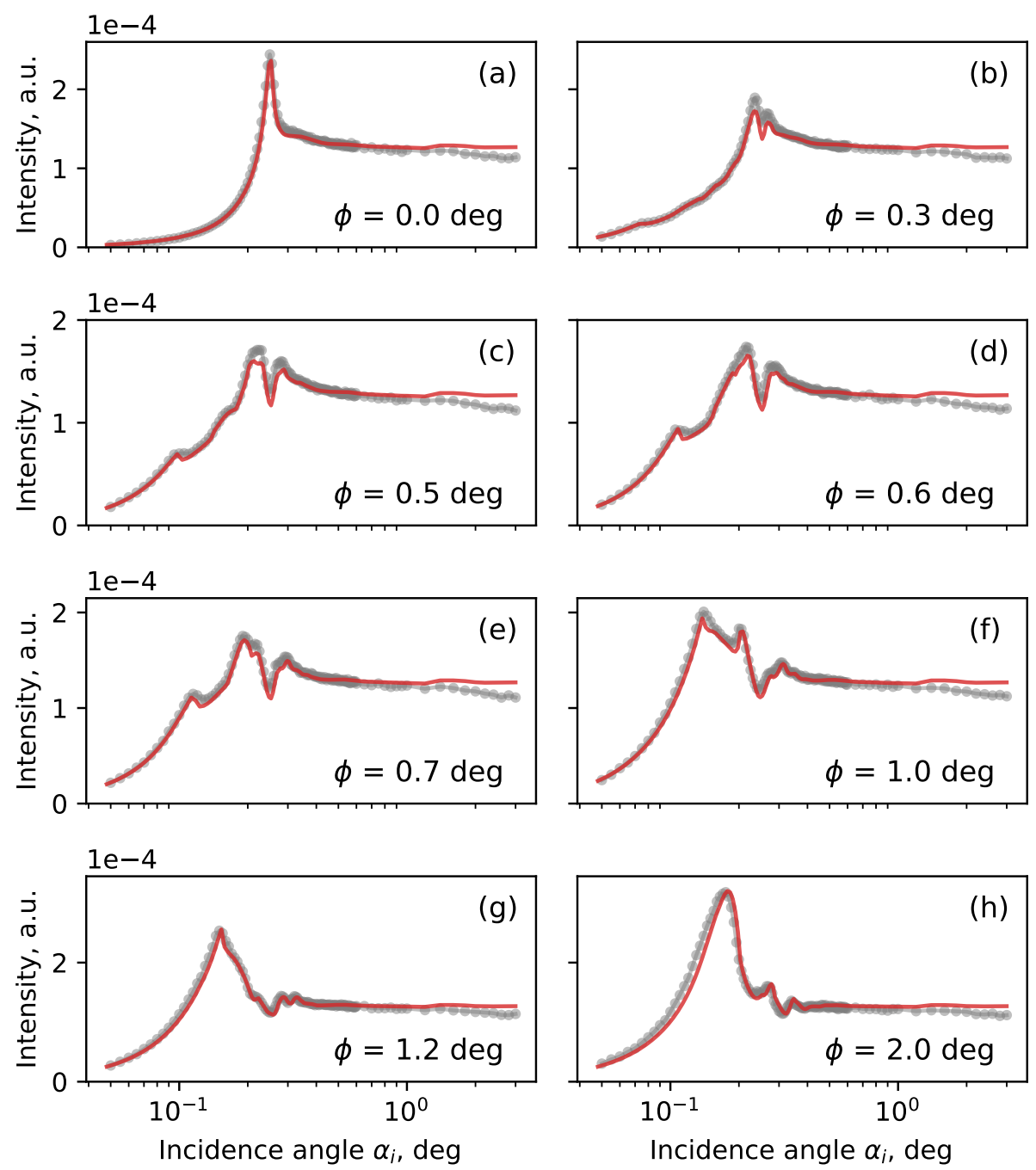

Figure 5.6: $\mathrm{Cr}-\mathrm{K} \alpha$ GIXRF intensity curves, measured for various azimuthal orientation angles (a) $\phi=0^{\circ}$ - conical (b) $\phi=0.3^{\circ}$, (c) $\phi=0.5^{\circ}$, (d) $\phi=0.6^{\circ}$, (e) $\phi=0.7^{\circ}$, (f) $\phi=1.0^{\circ}$, (g) $\phi=1.2^{\circ}$, and $(\mathrm{h}) \phi=2.0^{\circ}$. Red lines - numerical simulation, gray markers - experimental data.

GIXRF measurements were carried out at the four crystal monochromator (FCM) beamline [28] the PTB laboratory [23] of the BESSY II synchrotron radiation facility and reported by Dialameh et. al. [10]. The photon energy was $E=7 \mathrm{keV}$. Numerical simulations are done similarly to those in Section 5.3.1. GIXRF experimental data and the best-fit dynamical diffraction theory simulations are shown in Fig. 5.6, for a selection of azimuthal angles.

Best-fit model parameters are: lateral period of the structure $D x=D y=1 \mu \mathrm{m}$, 

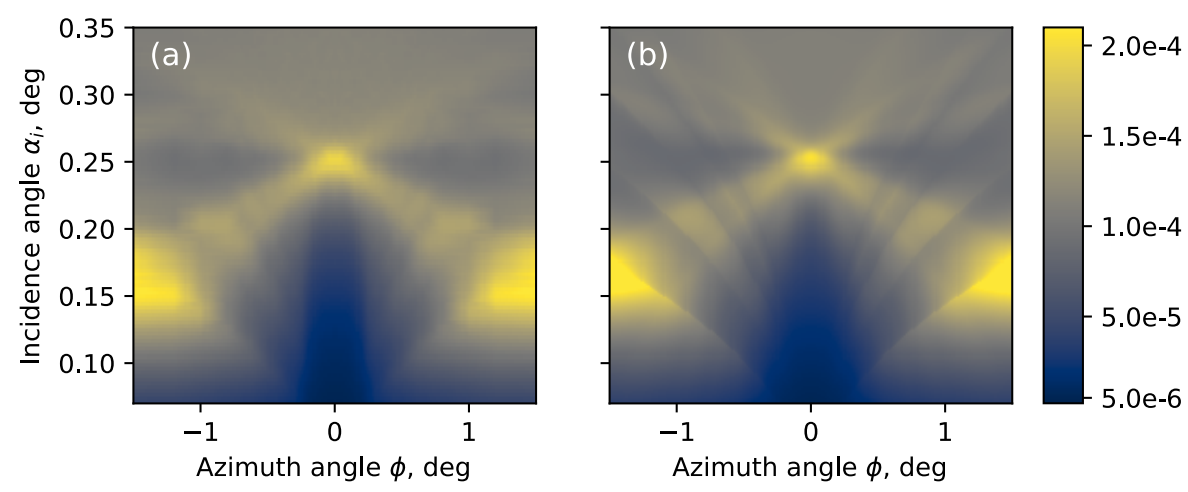

Figure 5.7: (3D XSW) Comparison between the experimental GIXRF map (a) of the Cr nanocolumn structure measured at incidence photon energy $E=7 \mathrm{kEv}$ and the simulated GIXRF map (b) based on a best fit model.

matching the same nominal values, lateral sizes of the nano-columns are $301 \mathrm{~nm} \times$ $301 \mathrm{~nm}$, nano-columns height $d=24 \mathrm{~nm}$ with effective depth of the surface oxidation $d_{0}=1.4 \mathrm{~nm}$, density of the material of nano-columns is nominal Cr density $\rho \approx$ $7.2 \mathrm{~g} / \mathrm{cm}^{3}$ Considering the large lateral period $D x=D y=1 \mu \mathrm{m}$ (significantly larger than that of $\mathrm{Si}_{3} \mathrm{~N}_{4}$ lamelar grating structure) the sidewalls tilt is negligible, therefore $\sigma=0 \mathrm{~nm}$, i.e. the best-fit model for the nano-column structure implies perfectly parallel sidewalls $\left\langle\chi_{h}\right\rangle \equiv \chi_{h}$. The interpolated experimental GIXRF map and the theoretical GIXRF map are shown in Fig. 5.7. Both experimental GIXRF curves Fig. 5.6 and experimental maps Fig. 5.7 are in good qualitative agreement with numerical simulations. Positions of the resonance lines in the experimental map and in numerical simulations in Fig. 5.7 match to the theoretical values calculated using Eq. 5.29 (see Fig. 5.8).

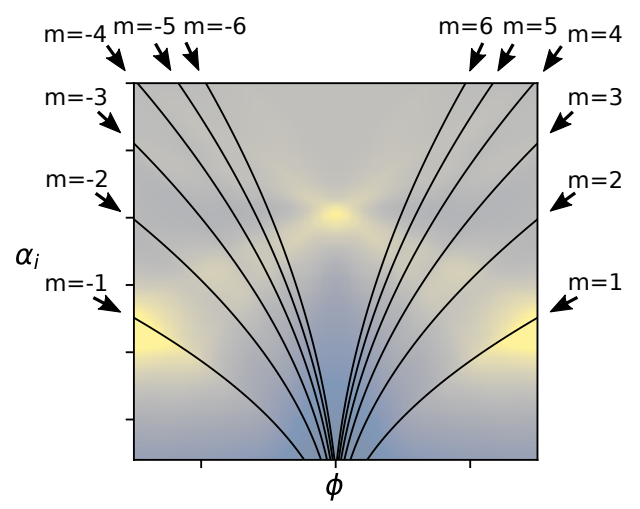

Figure 5.8: Resonant lines in GIXRF map for Cr nano-columns structure, caused by interference between reflected beam and $\mathrm{m}$-th order of diffraction. 


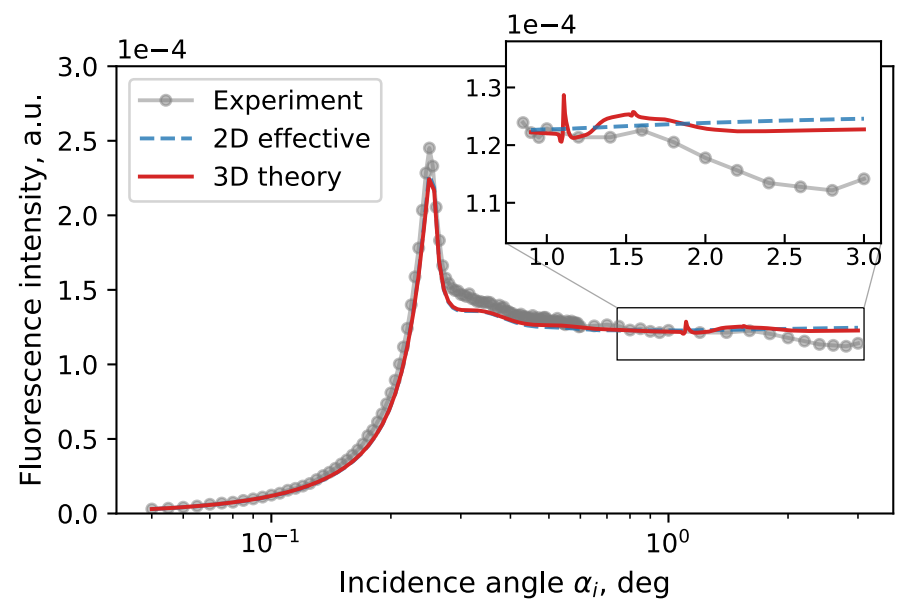

Figure 5.9: Comparison of effective 2D and genuine 3D simulations of GIXRF curve for 3D $\mathrm{Cr}$ nano-columns structure in conical geometry $\left(\phi=0^{\circ}\right)$.

It is important to note that in the case of grazing incidence geometry, the GIXRF curves calculated for the 3D structure could also be approximated with the use of an effective 2D model, albeit with reduced density. This is because in the grazing incidence geometry $\left|k_{y}\right| \gg\left|k_{x}\right|$. In other words, measurements in grazing incidence geometry are sensitive to the lower frequencies of the Fourier transform of the structure along the $x$ direction and to the higher frequencies along the $y$ direction, while the spacing between nodes in reciprocal space along $k_{x}$ and $k_{y}$ direction are identical due to the symmetry $D_{x}=D_{y}$ of the periodic structure.

Thus, GIXRF curves of Cr nano-columns can be effectively represented as a lamellar Cr grating with reduced density equal to the averaged density of the actual 3D structure. Indeed, GIXRF calculations with an effective 2D model and a genuine 3D model are qualitatively similar, see Fig. 5.9. However, there are clear differences between 2D and 3D calculations. For higher angles, the 2D model (dashed blue line in Fig. 5.9) yields a monotonous angular dependence, while the experimental GIXRF curve clearly exhibits oscillatory behaviour in that angular range, with a maximum at $\alpha_{i} \approx 1.5^{\circ}$. In Fig. 5.9, curves are shown only for $\phi=0^{\circ}$, but this oscillation in the range of higher incidence angles $\alpha_{i}$ is present in all experimental curves measured at different azimuthal orientations of the sample (see Fig. 5.6). We attribute this oscillation to interference due to the periodicity of the structure along the $y$ direction, which becomes more important at high incident angles since the value of $\left|k_{y}\right|$ decreases with increasing incidence angle $\alpha_{i}$ and the measurement becomes more sensitive to the lower frequencies of the Fourier transform along the $y$ direction. Such interference mode is not taken into account in the $2 \mathrm{D}$ simulations. Additionally, the $3 \mathrm{D}$ simulations show resonant peaks at $\alpha_{i} \approx 1.15^{\circ}$ and $\alpha_{i} \approx 1.54^{\circ}$ which are not resolved in experimental data. To observe these peaks, measurements with step sizes of $\delta \alpha_{i}=0.01^{\circ}$ should be resolved, which is near the resolution limit of modern synchrotron sample stage equipment. 


\subsection{Discussion}

In Table 5.1 we compare the structure parameters of the 2D lamellar $\mathrm{Si}_{3} \mathrm{~N}_{4}$ grating, as reconstructed from MBDDT simulations in Section 5.2.5, with those obtained from the FEM reconstruction from [12]. The nominal parameters used in fabrication of the grating are also shown in Table 5.1. Qualitatively, results of reconstruction by means of FEM and MBDDT are in good agreement.

Table 5.1: Comparison of the structure parameters of the $\mathrm{Si}_{3} \mathrm{~N}_{4}$ lamellar grating, as reconstructed by FEM and MBDDT with nominal parameters. Nominal structure parameters are also shown.

\begin{tabular}{l|c|c|c}
\hline & Nominal & FEM & MBDDT \\
\hline \hline Line height $d, \mathrm{~nm}$ & 90 & 87 & 89 \\
Line width, nm & 40 & 43 & 43 \\
Period $D_{x} \mathrm{~nm}$ & 100 & 100 & 100 \\
Effective top layer $d_{0}, \mathrm{~nm}$ & - & 3.5 & 1.5 \\
Side walls tilt $\alpha, \mathrm{deg}$ & - & 4 & 5 \\
Density $\rho, \mathrm{g} / \mathrm{cm}^{3}$ & 3.2 & 2.8 & 2.8 \\
\hline
\end{tabular}

One can note in Table 5.1 a small discrepancy between FEM and MBDDT in reconstruction of line height $d$ and the thickness of oxidized top layer $d_{0}$. However, when the full line height, including the oxidized top, is considered, both FEM and MBDDT consistently yield $d+d_{0}=90.5 \mathrm{~nm}$. Diffraction of X-rays under grazing incidence condition is sensitive to the structure of the surface [29]. Therefore, we assume that this discrepancy is due to the dynamical surface diffraction effects which are not taken into account within simple box model. We will discuss how these effects can be taken into account within MBDDT later in this section.

In Table 5.2 we compare the structure parameters of the $3 \mathrm{D} \mathrm{Cr}$ nanocolums, as reconstructed from MBDDT simulations in Section 5.2.5, with nominal parameters used in fabrication of the nano-columns. Reconstruction of parameters of this structure by means of FEM is not feasible due to the following reasons. $\mathrm{Cr}$ is a heavy element and therefore bigger incident photon energy is required to excite $\mathrm{CrK} \alpha$ fluorescence as compared to $\mathrm{N}$ from previous example. Additionally, compared to the previous example, the value of period of this structure is one order of magnitude higher. That in combination with $3 \mathrm{D}$ dimensionality of the sample increases the computational effort of FEM to such a level that it practically becomes inapplicable for this structure.

Comparing reconstructed and nominal parameters in Table 5.2, a good agreement is obtained, especially since for the current case only a simple box model is used for the numerical simulations to describe the distribution of fluorescent atoms in the structure. While the results presented here already demonstrate the potential of MBDDT as a tool for nanometrology of 2D and 3D structures, further improvements can be made to expand the area of application.

The optimization scheme for fitting numerical simulations to the experimental data can be improved by using a rigorous formalism described in [30]. In [30], equations for the calculation of Pearson's $\chi^{2}$ criterion for the simulteneous analysis 
Table 5.2: Comparison of the 3D structure parameters of the regularly arranged Cr nano-columns reconstructed by MBDDT with nominal parameters.

\begin{tabular}{l|c|c}
\hline & Nominal & MBDDT \\
\hline \hline Column height $d, \mathrm{~nm}$ & 25 & 24 \\
Column width, nm & 300 & 301 \\
Period $D_{x, y}, \mu \mathrm{m}$ & 1 & 1 \\
Effective top layer $d_{0}, \mathrm{~nm}$ & - & 1.4 \\
Density $\rho, \mathrm{g} / \mathrm{cm}^{3}$ & 7.19 & 7.2 \\
\hline
\end{tabular}

of rocking curves from different crystallographic planes of a single crystal has been derived. Similarly, $\chi^{2}$ can be calculated for the simultaneous analysis of GIXRF curves measured on different azimuthal orientation angles $\phi$. Further, to estimated the contribution of each GIXRF curve to the quality of the fit, one can use the covariation approach described in Chapter 2. That will also allow to estimate confidential intervals of the reconstructed parameters.

To take into account a structure with tilted sidewalls and surface oxidation, the model of a structure can be discretized along the $z$ direction according to [31]. The sample, can be approximated as a stack of homogeneous and/or structured layers, where each layer can have arbitrary structure parameters with the exception of period - period must be maintained throughout the whole stack. That restriction on period parameter does not limit the area of applications of such an approach, since by definition it is possible to find an elementary cell for each periodical structure.

The main interest in applications of 3D XSW technique is its proposed sensitivity to the inhomogeneous fluorescent atoms distribution within the structure. Although the examples considered in this work exhibited homogenious lateral distribution of $\mathrm{N}$ and $\mathrm{Cr}$ within the grating line and nano-column, an inhomogeneous distribution of fluorescent atoms in the lateral direction can be taken into account by selecting a polynomial function to approximate $p\left(\mathbf{r}_{\|}\right)$in Eq. 5.26. Approximation of $p\left(\mathbf{r}_{\|}\right)$ with a polynomial function is preferable since the integral in Eq. 5.26 can then be conveniently solved by parts.

To demonstrate the sensitivity of XSW to an an inhomogeneous distribution of fluorescent atoms, the simple box model can be used. Simulations were performed for a model of a $\mathrm{Si}_{3} \mathrm{~N}_{4}$ lamellar grating, similar as the one described in Section 5.3.1. However, here we consider the $\mathrm{N}$ atoms to be localized at a specific lateral or transversal location within the structure, instead of being homogeneously distributed, as assumed in Section 5.3.1. Figs. 5.10(a-c) and 5.11(a-c) show the assumed specific localization (green boxes) of $\mathrm{N}$ atoms, as well as the simulated GIXRF maps obtained for such localizations. In the simulations, the effect of the specific distribution of $\mathrm{N}$ atoms on the optical properties of the structure (i.e. on the intensity distribution of the XSW) is neglected, since the optical density of $\mathrm{N}$ is much lower than that of Si. In Fig. $5.10(\mathrm{a}-\mathrm{c})$, the range of distribution of fluorescent atoms is chosen such that it varies with respect to the center of the grating line. It can be observed that the corresponding GIXRF maps are highly sensitive to this variation. Only for the case of a symmetric distribution of fluorescent atoms 

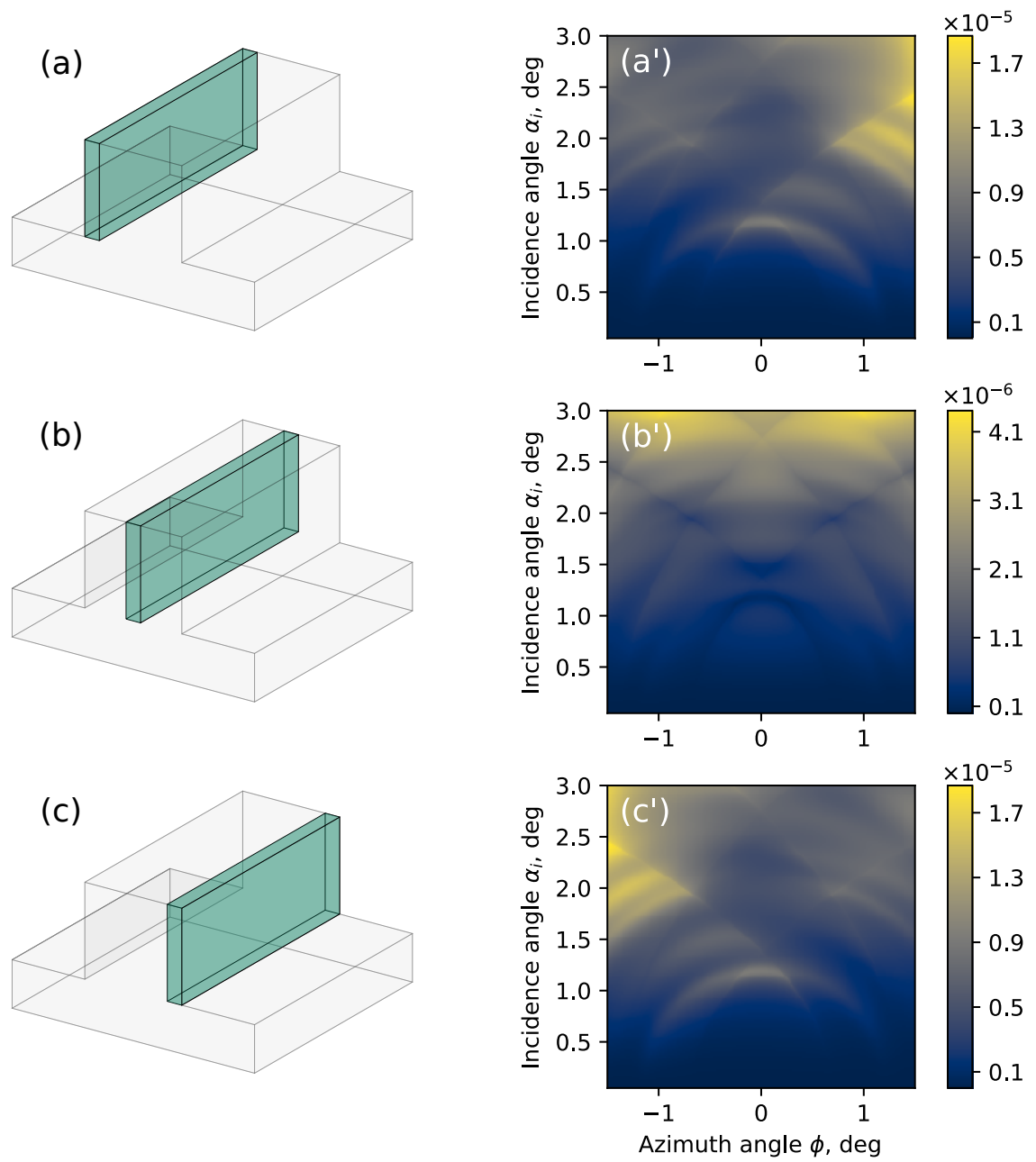

Figure 5.10: Simulation of GIXRF maps for inhomogeneous distribution of fluorescent atoms within the lamellar grating structure. From (a) to (c): sketch of the structure, green box depicts the localization of fluorescent atoms. From $\left(\mathrm{a}^{\prime}\right)$ to $\left(\mathrm{c}^{\prime}\right)$ : corresponding GIXRF map.

with respect to the center of the line Figs. 5.10(b), one obtains a symmetric GIXRF map (with respect to azimuthal angle phi). For "assymetric" distributions of fluorescent atoms, also an assymmetry is observed in the GIXR maps. One may exploit such asymmetry to distinguish chemical compositions of the left and right sidewall of the line. This might be useful in, e.g., the characterization of double-pattern gratings [32].

From Fig. 5.11(a-c), it can be observed that when the distribution of fluorescent $\mathrm{N}$ atoms is varied in depth, the resulting simulations of GIXRF show strong 

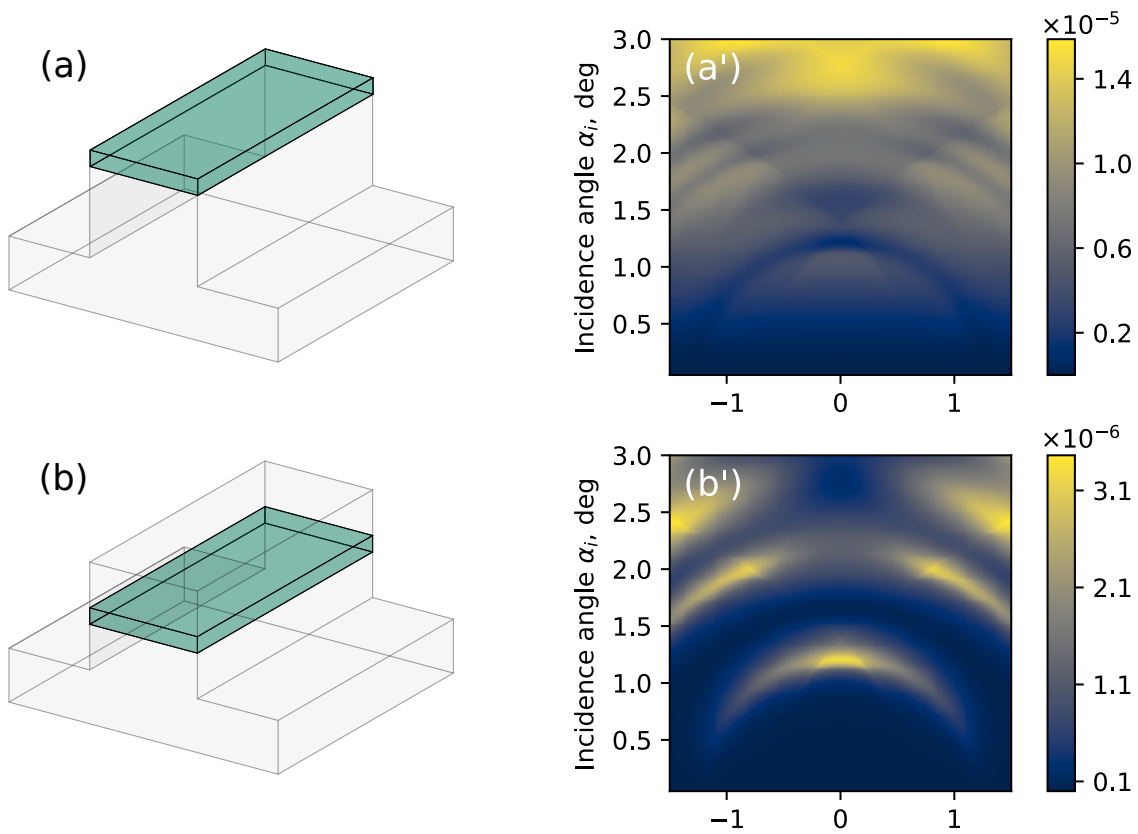

2.1

1.1
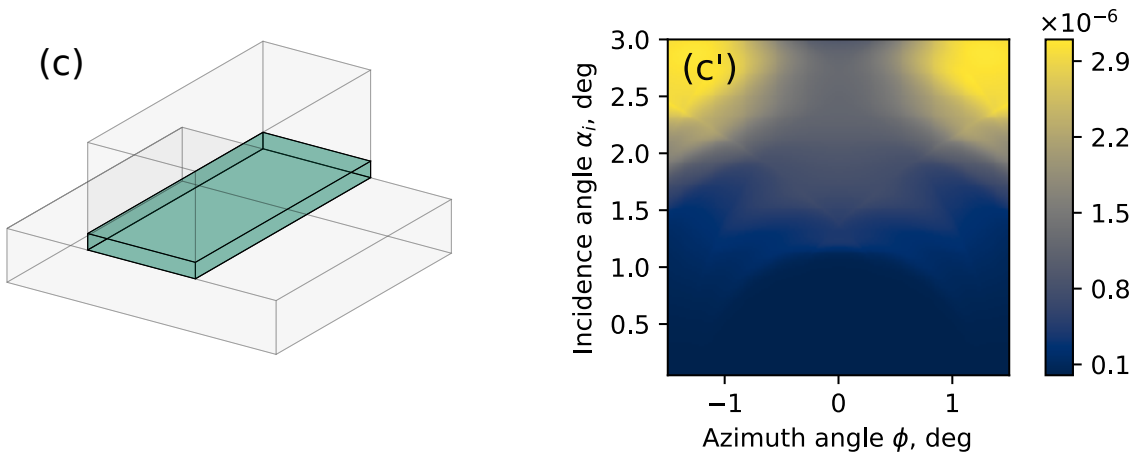

Figure 5.11: Simulation of GIXRF maps for inhomogeneous distribution of fluorescent atoms within the lamellar grating structure. From (a) to (c): sketch of the structure, green box depicts the localization of fluorescent atoms. From $\left(a^{\prime}\right)$ to $\left(c^{\prime}\right)$ : corresponding GIXRF map.

differences between the considered cases. Such differences are well resolvable under typical experimental conditions. This demonstrates the sensitivity of the technique to the depth distribution of fluorescent atoms.

It is important to note that while the analysis of GIXRF curves of Cr nanocolumns structure in Section 5.3.2 could also be performed with the use of an effective 2D model, in case of a strong asymmetry in lateral periodicity of the $3 \mathrm{D}$ structure $D_{x} D_{y}$, the use of an effective 2D model will fail. For the case of strong asymmetry, the effects of interference on periodical structure along $x$ and $y$ 
directions would strongly affect the GIXRF curves. The examples of such structures can be found in spectroscopic instruments for astrophysics [33, 34] or in EUV plasma source spectroscopy [35].

As a direction of further research, the MBDDT can be modified to take into account dispersions along the order of the structures in correlated 3D structures, using the formalism of para-crystal structures as it was done in Chapter 3 for the density fluctuations. Such computational scheme might find its implementation in highly correlated structures like quantum dots. Finally, XSW techniques can be implemented on lab-scale diffractometer [36] as opposed to the synchrotron source, making it widely accessible which is important for a nanometrological tool.

\subsection{Conclusions}

A new computational scheme based on the dynamical diffraction theory has been developed for the analysis of GIXRF experiments on 2D and 3D periodic structures. It is capable of simulating GIXRF data from structures with specific element distributions both in-plane as well as in-depth. The computational scheme has been benchmarked on GIXRF experimental data measured on $\mathrm{Si}_{3} \mathrm{~N}_{4} 2 \mathrm{D}$ lamellar gratings and $\mathrm{Cr}$ 3D nano-columns. Parameters of the elemental distribution in the $\mathrm{Si}_{3} \mathrm{~N}_{4} 2 \mathrm{D}$ lamellar grating reconstructed with the novel computational scheme are in good agreement with nominal values, as well as with parameters obtained from a reconstruction performed using a finite element method. Further, parameters of the elemental distribution in Cr 3D nano-columns were reconstructed for the first time. Results of this reconstruction are in good agreement with nominal parameters of the structure. We conclude that this computational scheme can be further developed and used as a powerful tool in element selective nanometrology for 3D periodic structures. 


\section{References}

[1] I. L. Markov, Limits on fundamental limits to computation, Nature 512, 147 EP (2014).

[2] E. Buitrago, R. Fallica, D. Fan, T. S. Kulmala, M. Vockenhuber, and Y. Ekinci, $\mathrm{SnO}_{x}$ high-efficiency EUV interference lithography gratings towards the ultimate resolution in photolithography, Microelectronic Engineering 155, 44 (2016).

[3] Z. Jörg and A. Kazimirov, X-ray Standing Wave Technique, The: Principles and Applications (Series on Synchrotron Radiation Techniques and Applications) (World Scientific Pub Co Inc, 2013).

[4] I. Kröger, B. Stadtmüller, C. Kleimann, P. Rajput, and C. Kumpf, Normalincidence X-ray standing-wave study of copper phthalocyanine submonolayers on $\mathrm{Cu}(111)$ and Au(111), Phys. Rev. B 83, 195414 (2011).

[5] S. N. Yakunin, I. A. Makhotkin, R. W. E. van de Kruijs, M. A. Chuev, E. M. Pashaev, E. Zoethout, E. Louis, S. Y. Seregin, I. A. Subbotin, D. V. Novikov, F. Bijkerk, and M. V. Kovalchuk, Model independent X-ray standing wave analysis of periodic multilayer structures, Journal of Applied Physics 115, 134303 (2014).

[6] N. N. Novikova, S. I. Zheludeva, O. V. Konovalov, M. V. Kovalchuk, N. D. Stepina, I. V. Myagkov, Y. K. Godovsky, N. N. Makarova, E. Y. Tereschenko, and L. G. Yanusova, Total reflection X-ray fluorescence study of langmuir monolayers on water surface, Journal of Applied Crystallography 36, 727 (2003).

[7] P. C. Fletcher, V. K. R. Mangalam, L. W. Martin, and W. P. King, Field emission from nanometer-scale tips of crystalline $\mathrm{PbZr}_{x} \mathrm{Ti}_{1-x} \mathrm{O}_{3}$, Journal of Vacuum Science \& Technology B 31, 021805 (2013).

[8] M. Malerba, A. Alabastri, E. Miele, P. Zilio, M. Patrini, D. Bajoni, G. C. Messina, M. Dipalo, A. Toma, R. Proietti Zaccaria, and F. De Angelis, 3D vertical nanostructures for enhanced infrared plasmonics, Sci Rep 5, 16436 (2015).

[9] G. P. Kennedy, O. Buiu, and S. Taylor, Oxidation of silicon nitride films in an oxygen plasma, Journal of Applied Physics 85, 3319 (1999).

[10] M. Dialameh, F. Ferrarese Lupi, P. Hönicke, Y. Kayser, B. Beckhoff, T. Weimann, C. Fleischmann, W. Vandervorst, P. Dubček, B. Pivac, et al., Development and synchrotron-based characterization of $\mathrm{Al}$ and $\mathrm{Cr}$ nanostructures as potential calibration samples for $3 D$ analytical techniques, physica status solidi (a) 215, 1700866 (2018).

[11] A. Gibaud and S. Hazra, X-ray reflectivity and diffuse scattering, Current Science $\mathbf{7 8 ,} 1467$ (2000). 
[12] V. Soltwisch, P. Hönicke, Y. Kayser, J. Eilbracht, J. Probst, F. Scholze, and B. Beckhoff, Element sensitive reconstruction of nanostructured surfaces with finite elements and grazing incidence soft X-ray fluorescence, Nanoscale 10, 6177 (2018).

[13] J. Pomplun, S. Burger, L. Zschiedrich, and F. Schmidt, Adaptive finite element method for simulation of optical nano structures, physica status solidi (b) 244, 3419 (2007).

[14] J. Sherman, The theoretical derivation of fluorescent X-ray intensities from mixtures, Spectrochimica Acta 7, 283 (1955).

[15] P. Hönicke, B. Beckhoff, M. Kolbe, D. Giubertoni, J. van den Berg, and G. Pepponi, Depth profile characterization of ultra shallow junction implants, Analytical and Bioanalytical Chemistry 396, 2825 (2009).

[16] P. Mikulík and T. Baumbach, X-ray reflection by rough multilayer gratings: Dynamical and kinematical scattering, Phys. Rev. B 59, 7632 (1999).

[17] R. M. Gray et al., Toeplitz and circulant matrices: A review, Foundations and Trends in Communications and Information Theory 2, 155 (2006).

[18] M. Born and E. Wolf, Principles of optics: electromagnetic theory of propagation, interference and diffraction of light (Elsevier, 2013).

[19] S. A. Stepanov, E. A. Kondrashkina, R. Köhler, D. V. Novikov, G. Materlik, and S. M. Durbin, Dynamical X-ray diffraction of multilayers and superlattices: Recursion matrix extension to grazing angles, Phys. Rev. B 57, 4829 (1998).

[20] B. Beckhoff, Reference-free X-ray spectrometry based on metrology using synchrotron radiation, J. Anal. At. Spectrom. 23, 845 (2008).

[21] W. Li, J. Zhu, X. Ma, H. Li, H. Wang, K. J. Sawhney, and Z. Wang, Geometrical factor correction in grazing incident $X$-ray fluorescence experiment, Review of Scientific Instruments 83, 053114 (2012).

[22] J. Lubeck, B. Beckhoff, R. Fliegauf, I. Holfelder, P. Hönicke, M. Müller, B. Pollakowski, F. Reinhardt, and J. Weser, A novel instrument for quantitative nanoanalytics involving complementary $X$-ray methodologies, Review of Scientific Instruments 84, 045106 (2013).

[23] F. Senf, U. Flechsig, F. Eggenstein, W. Gudat, R. Klein, H. Rabus, and G. Ulm, A plane-grating monochromator beamline for the PTB undulators at BESSY II, Journal of synchrotron radiation 5, 780 (1998).

[24] B. Beckhoff, A. Gottwald, R. Klein, M. Krumrey, R. Müller, M. Richter, F. Scholze, R. Thornagel, and G. Ulm, A quarter-century of metrology using synchrotron radiation by PTB in berlin, physica status solidi (b) 246, 1415 (2009). 
[25] L. Goray, W. Jark, and D. Eichert, Rigorous calculations and synchrotron radiation measurements of diffraction efficiencies for tender X-ray lamellar gratings: conical versus classical diffraction, Journal of Synchrotron Radiation 25, 1683 (2018).

[26] V. Soltwisch, A. Haase, J. Wernecke, J. Probst, M. Schoengen, S. Burger, M. Krumrey, and F. Scholze, Correlated diffuse X-ray scattering from periodically nanostructured surfaces, Phys. Rev. B 94, 035419 (2016).

[27] M. Altissimo, E-beam lithography for micro-/nanofabrication, Biomicrofluidics 4, 026503 (2010).

[28] M. Krumrey, Design of a Four-Crystal Monochromator Beamline for Radiometry at BESSY II, Journal of Synchrotron Radiation 5, 6 (1998).

[29] P. L. Cowan, Diffraction of evanescent X-rays: Results from a dynamical theory, Phys. Rev. B 32, 5437 (1985).

[30] M. A. Chuev, A. A. Lomov, and R. M. Imamov, Simultaneous analysis of double-crystal $X$-ray rocking curves from a set of crystallographic planes, Crystallography Reports 51, 178 (2006).

[31] M. Pisarenco, R. Quintanilha, M. G. M. M. van Kraaij, and W. M. J. Coene, Fast semi-analytical solution of Maxwell's equations in Born approximation for periodic structures, J. Opt. Soc. Am. A 33, 610 (2016).

[32] T. Weber, T. Käsebier, A. Szeghalmi, M. Knez, E.-B. Kley, and A. Tünnermann, High aspect ratio deep UV wire grid polarizer fabricated by double patterning, Microelectronic Engineering 98, 433 (2012).

[33] H. Bräuninger, H. Kraus, H. Dangschat, K. P. Beuermann, P. Predehl, and J. Trümper, Fabrication of transmission gratings for use in cosmic X-ray and XUV astronomy, Appl. Opt. 18, 3502 (1979).

[34] D. A. Schwartz, Invited review article: The Chandra X-ray observatory, Review of Scientific Instruments 85, 061101 (2014).

[35] S. Goh, H. Bastiaens, B. Vratzov, Q. Huang, F. Bijkerk, and K. Boller, Fabrication and characterization of free-standing, high-line-density transmission gratings for the vacuum UV to soft X-ray range, Opt. Express 23, 4421 (2015).

[36] I. A. Makhotkin, E. Louis, R. W. E. van de Kruijs, A. E. Yakshin, E. Zoethout, A. Y. Seregin, E. Y. Tereschenko, S. N. Yakunin, and F. Bijkerk, Determination of the density of ultrathin la films in $\mathrm{La} / \mathrm{B}_{4} \mathrm{C}$ layered structures using $X$-ray standing waves, physica status solidi (a) 208, 2597 (2011). 



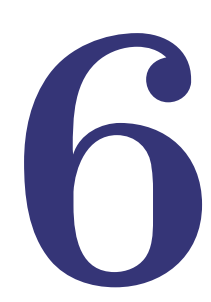

\section{EUV NEAR FIELD \\ CALCULATION FOR}

\section{NANO-SCALE GRATINGS}

In this work we compare calculations of the near field in nano-scale $2 D$ periodic structures in the EUV wavelength range done by dynamical diffraction theory, with calculations done using a finite element method. We found that near field calculations based on the dynamical diffraction theory are on the same level of accuracy as finite element methods but orders of magnitude faster. The observed gain in simulation speed is an essential step towards solving the inverse problem of analysis of grating structures by fitting simulations to measurement data.

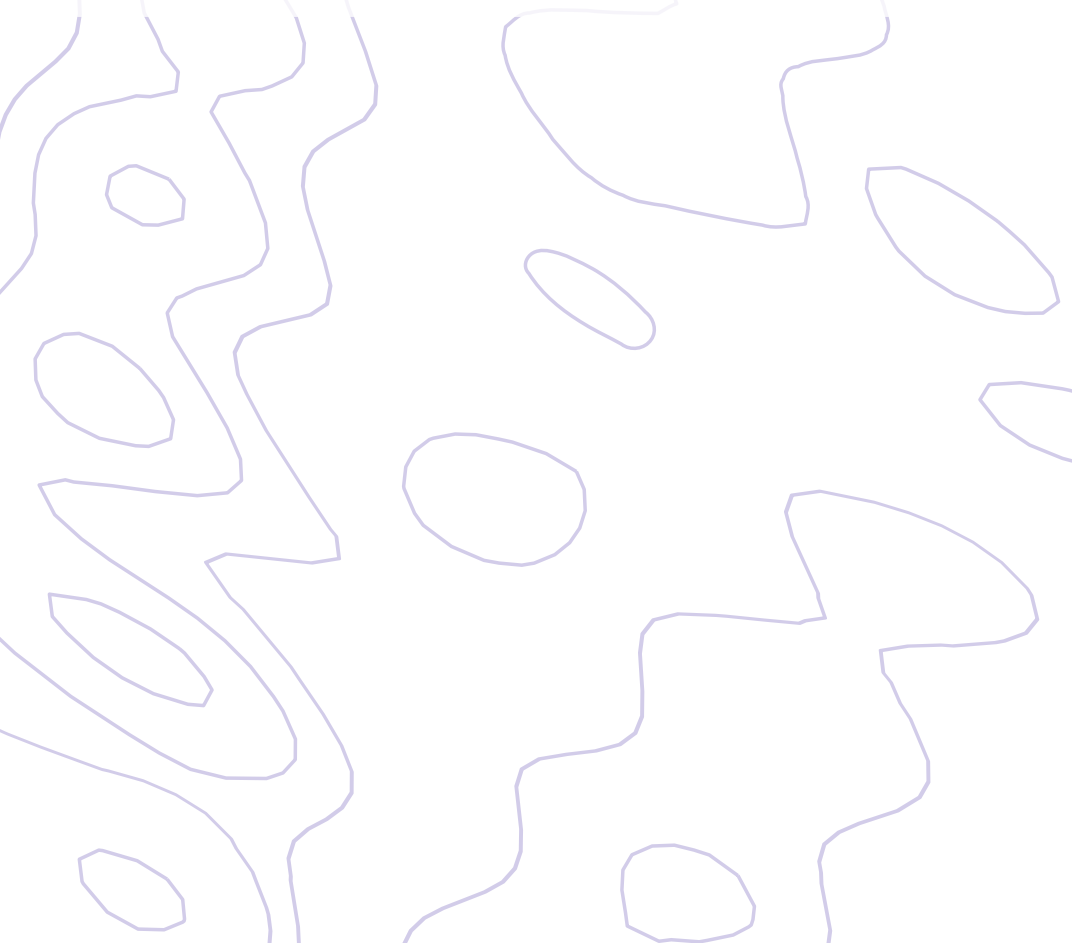




\subsection{Introduction}

Nano-scale 3D structures have a wide range of applications, from integrated circuits [1] (such as random access memory cells [2]) and semiconductor optoelectronic devices [3] to transmission gratings for UV and soft X-ray spectroscopy [4]. Recently, in particular due to increased demand from the semiconductor industry, EUV and X-ray scattering methods have been employed to non-destructively characterize such structures. These methods provide information on imperfections in the structures which affect their performance [5-7].

EUV and X-ray scattering can be simulated using finite element methods (FEM), such as such as the FEM based Maxwell solver JCMSuite [8]. Finite element methods are highly accurate, but when the ratio of the wavelength to the structure size becomes small they are computationally expensive (in both time and memory), as a finer mesh is required to deal with such structures. A semianalytical theory that retains the high accuracy and significantly reduces computational expense is therefore sought after for practical measurement data reconstruction [9].

Various semianalytical dynamical theories exist for the numerical calculation of diffraction efficiencies. The earliest such theory is the rigorous vector theory [10] which employs the Rayleigh expansion to express the electric field as a sum of spherical waves. However, the Rayleigh expansion is not always valid depending on the grating and region of interest (in particular in the near field it may fail) and therefore the rigorous vector theory is limited in its applicability. The differential method uses a Fourier series for both the electric field and susceptibility. The Fourier series is more often applicable than the Rayleigh expansion, and can easily be adapted to non-rectangular grating profiles as well. However, the differential method only takes into account dynamical interaction between diffraction orders at interfaces, but neglects interaction during propagation through a layer [11]. Dynamical diffraction theory also uses a Fourier series expansion for the electric field, and additionally takes into account dynamical interaction during propagation in between layer interfaces [10].

Dynamical diffraction theory in many-beam approximation (MBDDT) [12-14], also known as rigorous coupled wave analysis (RCWA), was originally developed for electron diffraction [15] and later for X-ray diffraction of crystal lattices [16] and gratings [17]. The theory was extended to blazed gratings [12] and to multilayer gratings [13]. To the best of our knowledge, its accuracy has so far not been tested in the near field nor for gratings azimuthally rotated with respect to the incident beam.

In this work, simulations by MBDDT and a Maxwell solver based on the finite element method are compared for the case of gratings in the EUV regime. We compare simulations of both the far field (diffraction efficiencies) and the near field. The good agreement between MBDDT simulation and reference FEM simulations makes MBDDT an excellent candidate for further development. For completeness, an efficient linear-algebraic formalism for calculation of the near field is presented and the dispersion surface calculated for the structured layers is examined, to study the diffraction process. Details are described in Appendix 6.4. 


\subsection{Numerical simulations}

In this section the numerical simulations performed using the dynamical diffraction theory are presented. The MBDDT simulations were compared to reference simulations using JCMSuite. JCMSuite is established as a reliable FEM package for rigorous numerical simulation of EUV and X-ray scattering from laterally extended gratings in the EUV regime [9, 18, 19].

Table 6.1: Parameters of the models used in simulations. Model 1 - grating with parallel sidewalls. Model 2 - lines with trapezoidal profile.

\begin{tabular}{c|c|c}
\hline & Model 1 & Model 2 \\
\hline \hline$d, \mathrm{~nm}$ & 90 & 90 \\
\hline$t, \mathrm{~nm}$ & 100 & 100 \\
\hline$L 1, \mathrm{~nm}$ & 45 & 35 \\
\hline$L 2, \mathrm{~nm}$ & 45 & 45 \\
\hline
\end{tabular}

We performed calculations of the diffraction efficiencies and the near field under the conditions of an s-polarized incident beam of wavelength $\lambda=13.5 \mathrm{~nm}$, incidence angles from $0^{\circ}$ to $50^{\circ}$ (from the surface), for two models: a rectangular profile (model 1) and a trapezoidal profile (model 2), see Table 6.1 and Fig. 6.1 for specifications.

We include a trapezoidal model as this type of gratings are often used rather than rectangular gratings $[9,18,19]$. In order to represent the tilted sidewalls, the discretization along the $z$ axis is used [20]. Thus, a slab of a non-rectangular profile is divided into multiple thin sublayers with continuously increasing width of the sublayers along $z$ axis. This model approximates a non-rectangular profile. (see Fig. 6.1b,c for the profiles, Table 6.1 for the specifications). In simulations, 20 structured layers were used as at this number of layers the diffraction efficiencies converged, i.e. adding more layers did not significantly change the simulation results.
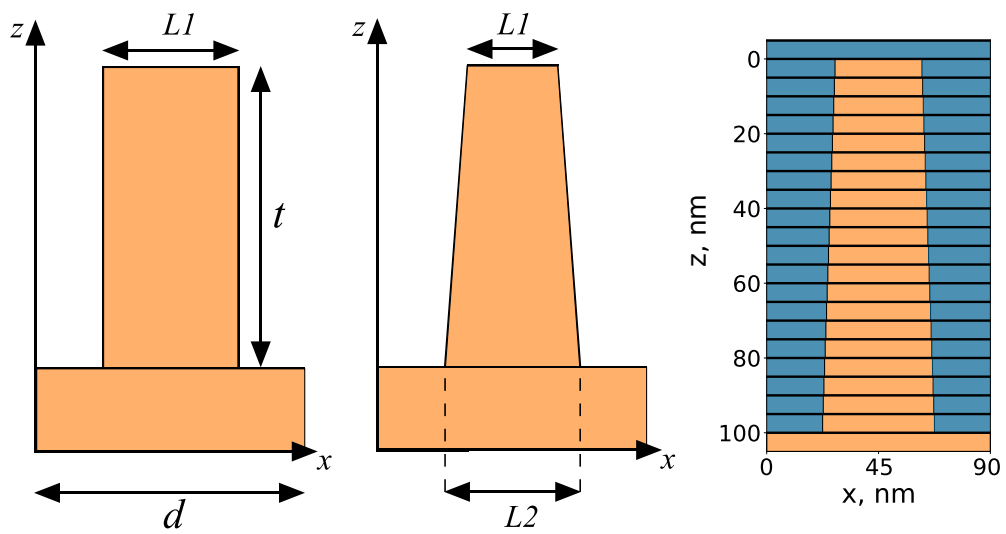

Figure 6.1: Model profiles, (a) model 1 and (b) model 2, ideal profile (as used in JCMSuite) and (c) sliced profile (20 structured layers, as used in MBDDT). 
(a)

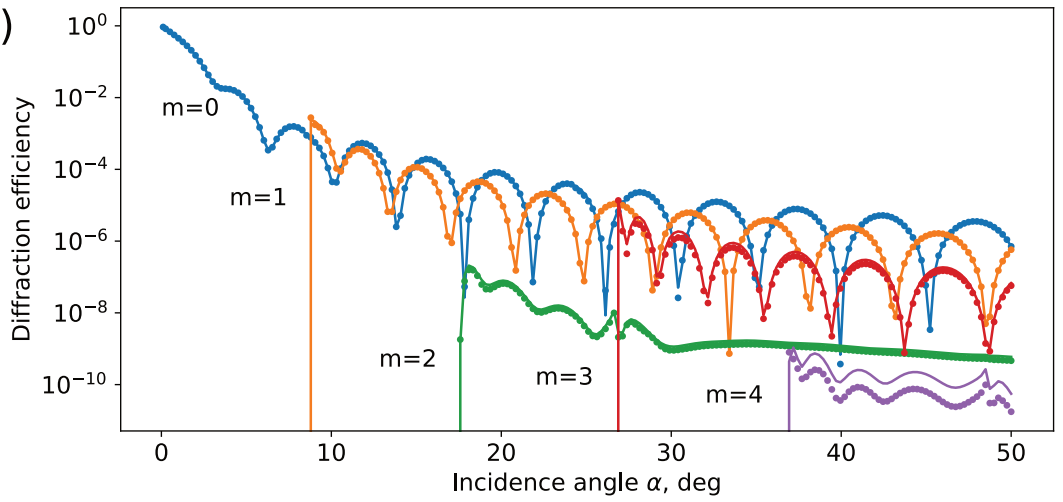

(b)
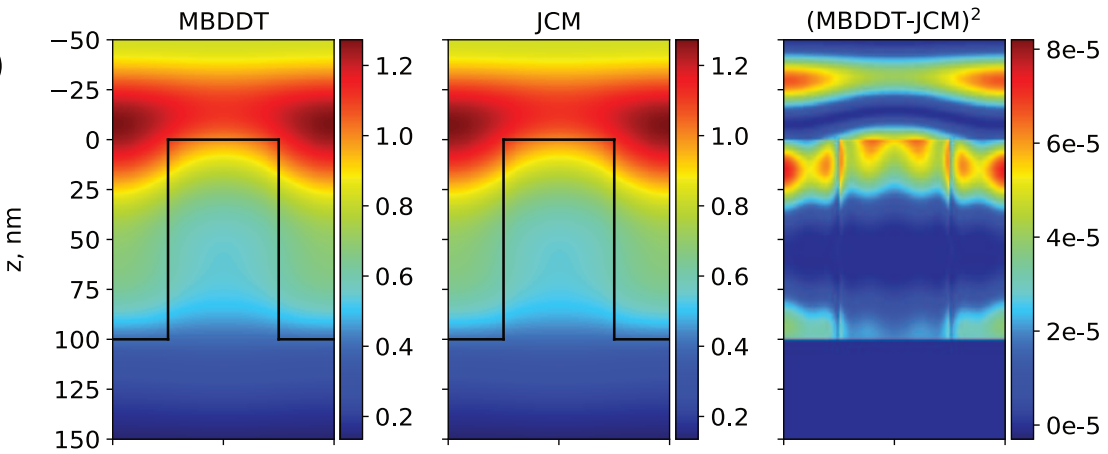

(c)
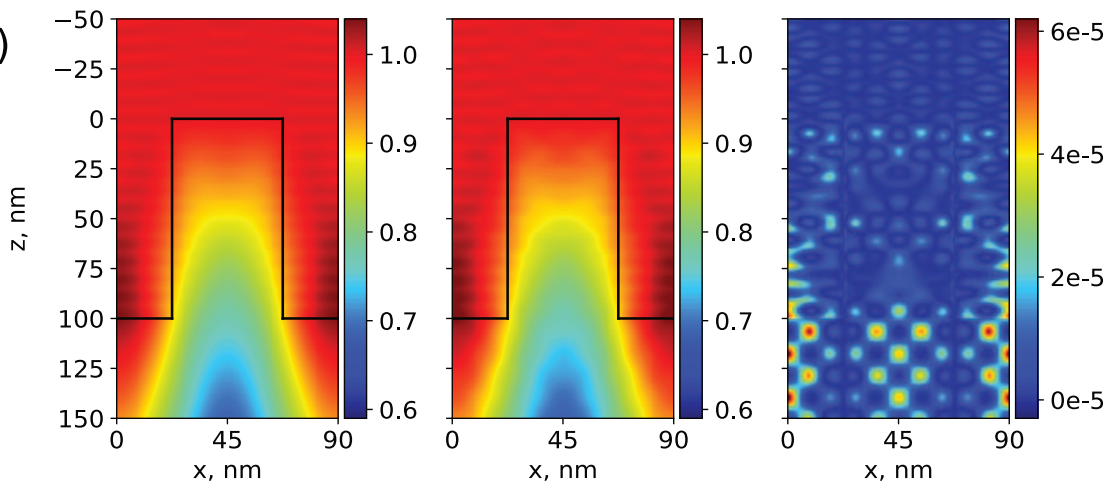

Figure 6.2: Model 1, parallel geometry, (a) diffraction efficiencies (lines from MBDDT and dots from JCMSuite), (b) and (c) near field (crosssection along the periodic direction) for incidence angle $5^{\circ}$ and $50^{\circ}$, respectively. MBDDT and JCM intensities are drawn using the same colorscales.

Both model 1 and model 2 are composed out of Silicon with electrical susceptibility $\chi=(-1.998+i 3.656) \cdot 10^{-3}$. Both models are simulated for parallel geometry (incident beam parallel to the $y$ axis) and model 1 is also simulated for an azimuthal rotation of $\phi=30^{\circ}$ with respect to conical geometry. The diffraction efficiencies and the near field simmulations for model 1 are shown in Figs. 6.2 and 6.4. The diffrac- 


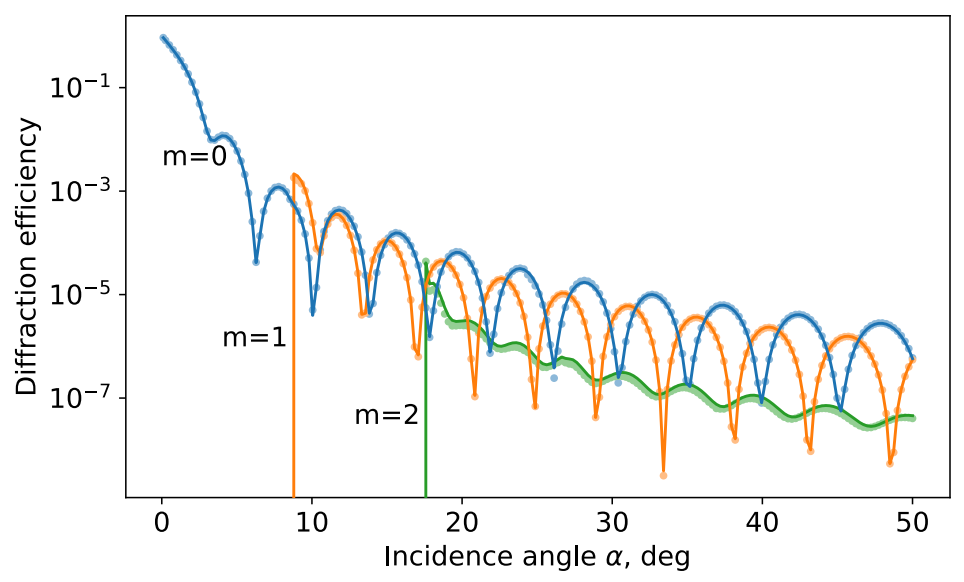

Figure 6.3: Model 2, diffraction efficiencies (lines from MBDDT and dots from JCMSuite).

tion efficiencies simulated for model 2 are shown in Fig. 6.3 The near field plots uses the same color scale for both, MBDDT and FEM simulations. The results for model 1 in conical geometry show excellent agreement between the FEM and MBDDT results. The near field is closely reproduced for both grazing and nongrazing incidence. The diffraction efficiencies are also correctly reproduced, though some of the higher orders (with reflectance values well below $10^{-8}$ ) are overestimated (in particular $m=3,4$, but the effect can already be noticed for $m=2$ ). The diffraction efficiencies for model 2 show the same behaviour: the curves from JCMSuite are reproduced, but the higher order $m=2$ is overestimated. However, for both models the modulations are correctly reproduced, as are the critical angles of each order.

The modulations are the result of interference between the wave modes, which depends on the phase of the field. As the phase of the field is correctly reproduced, so are the modulations. The overestimation of the intensity is likely due to the following assumption made in MBDDT: absorption is only taken into account for propagation along the $z$ axis - through the imaginary components of the wave vector components $k_{z n}$ - but neglected for propagation in the $x, y$ plane $-k_{x}$ and $k_{y}$ are always taken as real numbers. The finite element method does take into account this absorption and therefore results in slightly lower intensities. As for higher diffraction orders the path through the grating is longer (at least in conical geometry), the difference due to missing absorption terms is larger, as can been seen in the diffraction efficiencies. This could possibly be corrected by introducing the phase change due to this absorption into the propagation matrix $\mathbf{Q}^{(j)}$.

The results for model 1 azimuthally rotated with respect to the incident beam are shown in figure Fig. 6.4. The shape of the near field is reproduced, even though the magnitude is overestimated by the MBDDT. The features (modulations and critical angles) of the diffraction efficiencies are again well reproduced. 
(a)
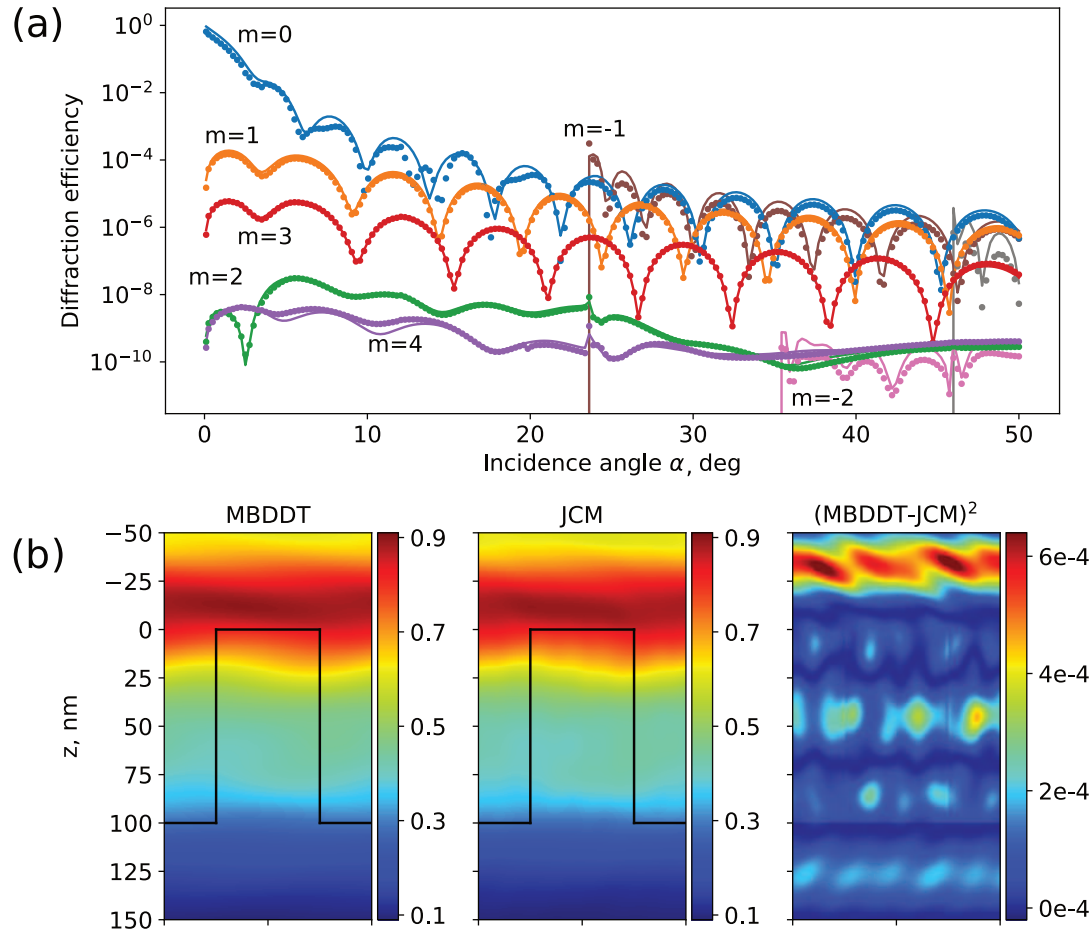

(c)
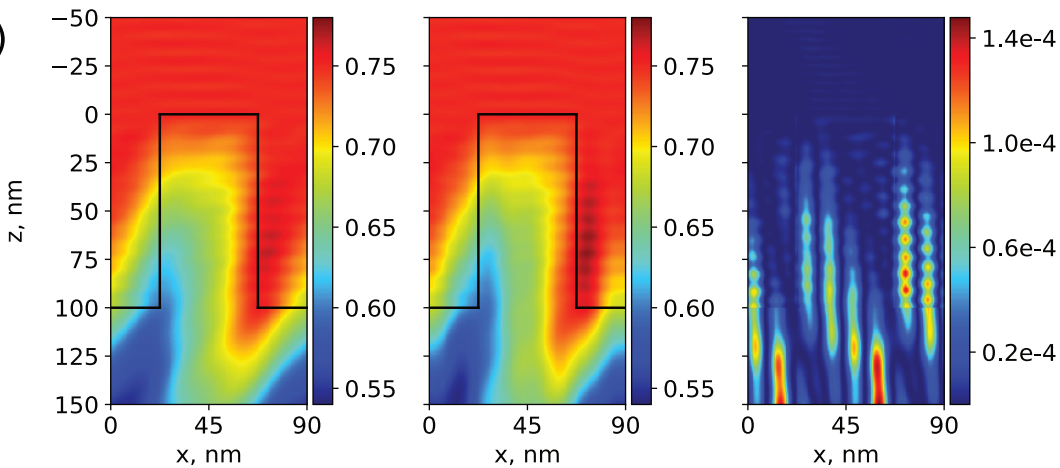

Figure 6.4: Model 1, $\phi=30^{\circ}$ azimuthal rotation, (a) diffraction efficiencies (lines from MBDDT and dots from JCMSuite), (b) and (c) near field (crosssection along the periodic direction) for incidence angle $5^{\circ}$ and $50^{\circ}$, respectively, intensity $I=|\vec{E}|^{2}$. MBDDT and JCM intensities are drawn using different colorscales.

\subsubsection{Dispersion surface}

The eigenvalues $k_{z n}^{(j)}$, together with the parallel components of the wave vectors $\vec{k}_{m \|}$ form the dispersion surface of $j$-th layer. The dispersion surface is used in detailed analysis of the diffraction process [21-23]. For the case of multilayer gratings, 

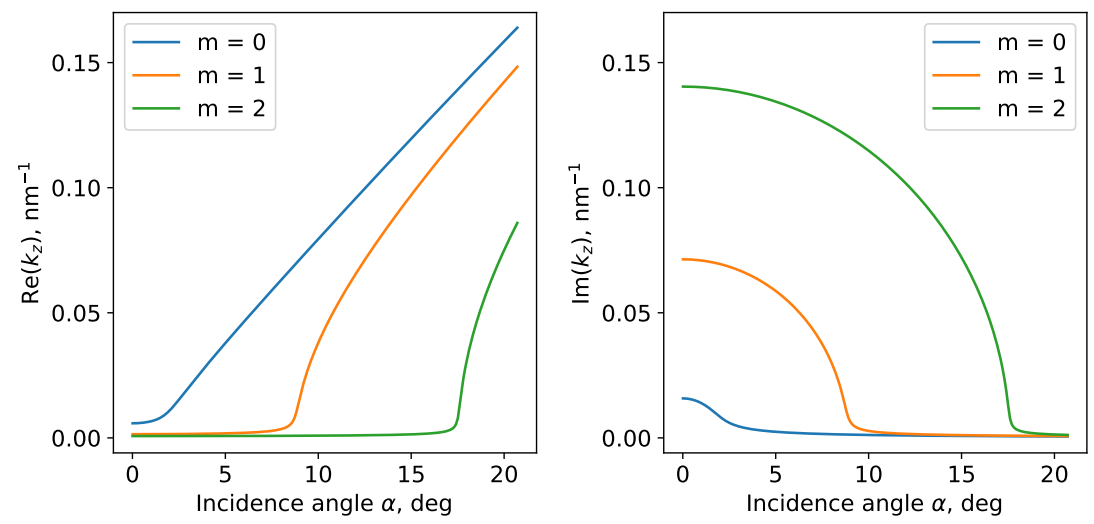

Figure 6.5: First three eigenvalues $k_{z n}$ calculated for the structured layer in model 1, parallel geometry.

plotting of the dispersion surface also links different eigenvalues $k_{z n}$ to orders of diffraction $m$. Finite element methods do not provide access to the dispersion surface, so this is an advantage of MBDDT over FEM.

In particular the eigenvalues $k_{z n}$ are of interest here. The real part determines - together with the predetermined $\vec{K}_{m \|}$ - the direction of the travelling wave inside the grating structure, while the imaginary part accounts for absorption. One can define the penetration depth $L_{n}$ as the depth at which the amplitude of a mode $n$ has decreased by a factor of $e$. Then the penetration depth is $L_{n}=1 / \operatorname{Im}\left(k_{z n}\right)$.

In Fig. 6.5 the first three eigenvalues as calculated for the structured layer by MBDDT for model 1 are shown. For each mode, the transition from evanescent wave to travelling wave occurs at the same angle as the associated diffraction efficiency becomes nonzero (see Fig. 6.2a. Below this angle, the real part is close to zero while the imaginary part increases, i.e. an evanescent wave with a decreasing penetration depth.

In addition to providing physical insight into the diffraction process, examination of the eigenvalues also allows selection of the right number of modes $\mathcal{D}$ to include in the simulation. Inclusion of modes $n$ with a penetration depth $L_{n}$ smaller than the characteristic size of feature of interest is unnecessary. Including many evanescent modes can even lead to numerical precision issues, and also slows down the simulations. In particular, the matrix multiplication used to solve equations for the amplitudes of different wave modes $\vec{T}$ and $\vec{R}$ (see Eq. 6.26) slows down significantly with an increase of modes $\mathcal{D}$.

\subsubsection{Comparison to scalar formulation}

Here we aim to compare the vector formalism used in the MBDTT simulations presented in this work, to the previous forms of MBDDT applied to (multilayer) gratings which used scalar approximation. Previous studies start from a scalar wave equation [13, 14, 24], whereas in this work the full vector wave equation is used (see 


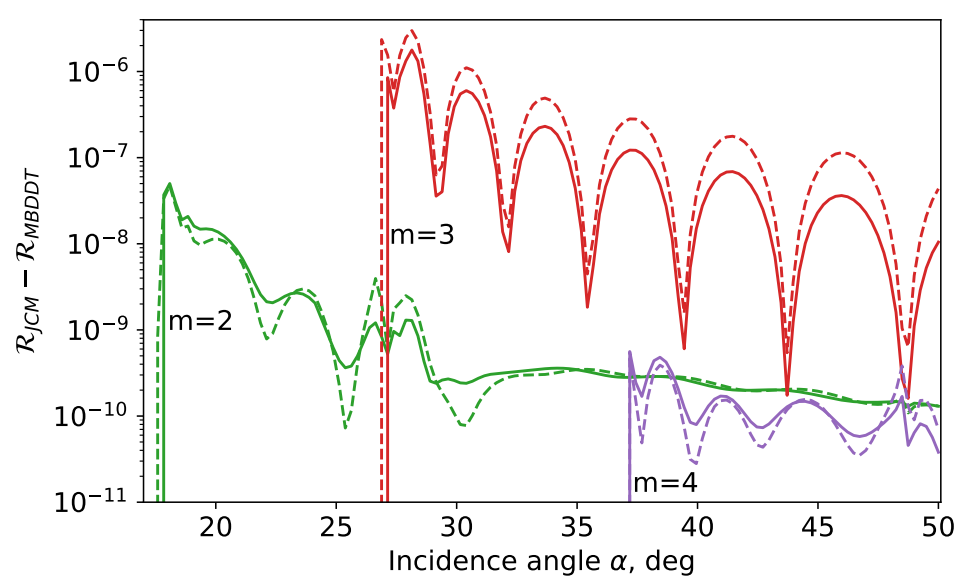

Figure 6.6: Model 1 (parallel geometry), residuals of diffraction efficiencies, JCMSuite minus scalar MBDDT (dashed lines) and JCMSuite minus vector MBDDT (full lines).

Eq. 6.8), which more accurately incorporates multiple beam interactions. The scalar wave equation leads to a different formulation of the eigenvalue problem (see Eq. 28 in [13])

$$
\left(\kappa_{z m}^{2}-k_{z n}^{2}\right) E_{m n}+\sum_{l \neq m} \chi_{m-l} E_{l n}=0
$$

while the vector formalism leads to Eq. 6.18:

$$
\left(\kappa_{z m}^{2}-k_{z n}^{2}\right) E_{m n}+\sum_{l \neq m} \chi_{m-l} E_{l n} \vec{e}_{m} \cdot \vec{e}_{l}=0
$$

The difference is in the term $\vec{e}_{m} \cdot \vec{e}_{l}$, which increases the accuracy of the multiple beam interaction. Note that if $\vec{e}_{m} \cdot \vec{e}_{l}=1$ the model reduces to the scalar case. In Fig. 6.6, the differences (residuals) between diffraction efficiencies calculated using scalar/vector forms of MBDDT and those calculated using JCMSuite are shown for model 1 are shown.

It is interesting to note that for the odd order, the MBDDT residuals show the same shape and the vector form is only slightly more accurate (the residuals curve is almost the same but slightly smaller) while for the even orders the vector form much more closely follows the shape of the JCMSuite curve (the residuals oscillate less, indicating a closer replication of the JCMSuite curve). The physical reason for this is that for model 1 the grating profile is half a period wide, so all even diffraction orders are suppressed due to destructive interference. That these orders are excited is entirely due to the multiple beam interaction. Therefore the even orders are more strongly affected by the vector formulation improvement to the interaction terms. 


\subsection{Conclusions}

In this work, we have shown that dynamical diffraction theory in many-beam approximation (MBDDT) shows good agreement with FEM based simulations (JCMSuite) in simulating diffraction on grating structures. MBDDT reproduces results for diffraction efficiencies and near field for parallel geometry and azimuthally rotated models in the EUV regime. Non-rectangular profiles can be simulated using slicing without loss of accuracy. The vector MBDDT formalism considered in this work more accurately accounts for the interference between different diffraction orders, improving accuracy of diffraction efficiency simulations in cases where many beam interaction have a significant role, such as critical angle regions and otherwise suppressed orders of diffraction.

\subsection{Appendix}

The derivation of multiple beam dynamical diffraction theory presented here is largely based on the work by Mikulík and Baumbach [13]. The most distinguishing difference is that we start from the vector wave equation, rather than the scalar wave equation. Also, we present a linear algebra formalism for efficient calculation of the near field.

\subsection{A The parametrization of test samples and geometry of the simulations}

Here we consider multilayer gratings of $N$ periodic layers deposited on a substrate (see Fig. 6.7). Therefore there are $N+1$ horizontal interfaces, labelled 0 through $N$. The vacuum above the grating is layer 0 , and the vacuum interface is at $z_{0}=0$, and the substrate is located interface at $z_{N}$. We consider here the ideal structure, with

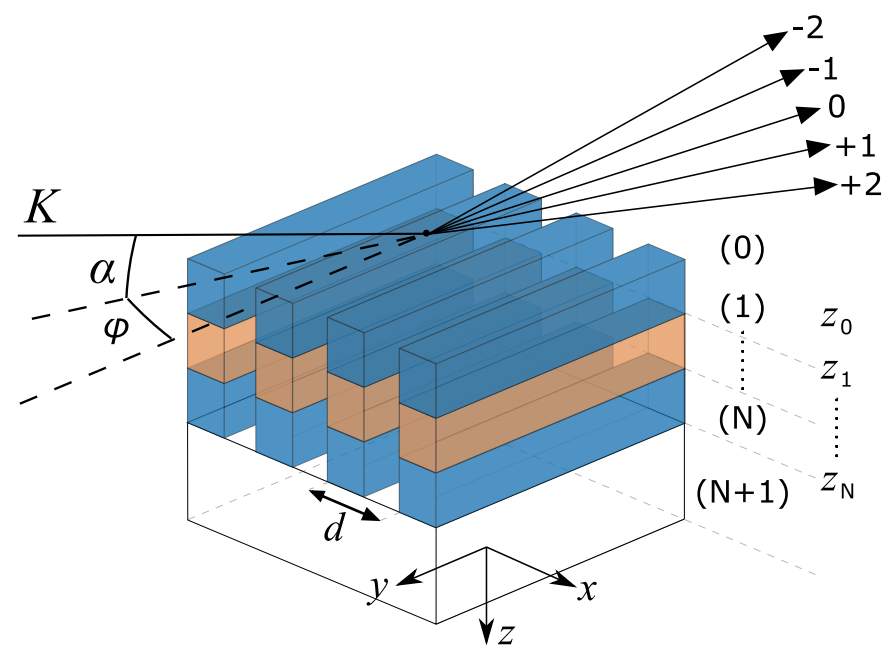

Figure 6.7: Schematic representation of scattering geometry of a sliced grating model. 
a sharp interface $j$ between every pair of layers at $z=z_{j}$. A layer can either consist of one material with a constant susceptibility (homogeneous layer) or it can consist of multiple materials with a susceptibility that is periodic along $x$ (structured layer) with period $d$. This allows expansion of the susceptibility $\chi(x, z)$ into a Fourier series in each layer $j$ :

$$
\chi(x)=\sum_{m} \chi_{m} e^{i h_{m} x}
$$

with Fourier coefficients:

$$
\chi_{m}=\frac{1}{d} \int_{0}^{d} \chi(x) e^{-i h_{m} x} d x
$$

Here $h=\frac{2 \pi m}{d}$ is the grating vector of order $m \in \mathbb{Z}$. We analytically evaluate the integral for the Fourier coefficients $\chi_{m}$ for the case where $\chi(x)$ is a sequence of $b$ rectangular profiles:

$$
\chi_{m}=\frac{1}{d} \int_{0}^{d} \chi(x) e^{-i h_{m} x} d x=\sum_{a=1}^{b} \chi_{m, a}
$$

with

$$
\chi_{m, a}=\frac{\chi_{a}}{d} \int_{x_{a}}^{x_{a+1}} e^{-i h_{m} x} d x .
$$

The evaluation of the integral depends on the value of $h_{m}$ :

$$
\chi_{m, a}= \begin{cases}\frac{\chi_{a}}{d}\left(x_{a+1}-x_{a}\right) & \left(h_{m}=0\right) \\ \frac{i \chi_{a}}{d h_{m}}\left[e^{-i h_{m} x_{a+1}}-e^{-i h_{m} x_{a}}\right] & \left(h_{m} \neq 0\right)\end{cases}
$$

where $\chi_{a}$ is the value of the susceptibility from $x_{a}$ to $x_{a+1}$ and $x_{0}=0, x_{b}=d$.

\subsection{B Dynamical solution to the wave equation}

Maxwell's equations describe the propagation of X-rays through gratings. In the case of the plane wave approximation, they reduce to:

$$
\left(K^{2}+\nabla^{2}\right) \vec{E}(\vec{r})+K^{2} \chi(\vec{R}) \vec{E}(\vec{r})=0 .
$$

We expand the electric field around the incident beam wave vector on the same reciprocal space lattice as the susceptibility:

$$
\begin{gathered}
\vec{E}(\vec{r})=\sum_{m} \vec{e}_{m} e^{i \vec{k}_{m \| \mid} \vec{r}_{\|}} E_{m}(z), \\
\vec{k}_{m \|}=\vec{K}_{\|}+\vec{h}_{m}=\left(\begin{array}{c}
K_{x}+h_{m} \\
K_{y} \\
0
\end{array}\right) .
\end{gathered}
$$


Here $\vec{e}_{m}$ is the unit vector pointing in the direction of the electric field wave mode $m$. We then define

$$
\kappa_{z m}=\sqrt{\left(1+\chi_{0}\right) K^{2}-\vec{k}_{m \|}^{2}}
$$

the wave vector $z$-component according to spherical dispersion (taking into account the change in wavelength due to refraction and the continuity of the parallel wavevector components). Note that the $z$-components are different in each layer (depending on the average susceptibility of the layer $\chi_{0}$ ) while the parallel components $\vec{k}_{m \|}$ are continuous as is required by the boundary conditions on the electric field.

We substitute these forms of $\vec{E}(x, y)$ and $\chi(x)$ into the wave equation to search for the $E_{m}(z)$ that solves it:

$$
\begin{aligned}
\sum_{m}\left(K^{2}-\vec{k}_{m \|}^{2}+\frac{d^{2}}{d z^{2}}\right) \vec{e}_{m} E_{m} & (z) e^{i \vec{k}_{m \| \mid} \cdot \vec{r}} \\
& +K^{2}\left[\sum_{m} \chi_{m} e^{i h_{m} x}\right]\left[\sum_{m} e^{i \vec{k}_{m \|} \vec{e}_{m}} E_{m}(z)\right]=0 .
\end{aligned}
$$

Here, $\chi_{0}$ can be taken out of the sum, so that we can introduce the spherical dispersion components from Eq. 6.11:

$$
\begin{aligned}
& \sum_{m}\left(\kappa_{z m}^{2}+\frac{d^{2}}{d z^{2}}\right) \vec{e}_{m} E_{m}(z) e^{i \vec{k}_{m \| \mid} \cdot \vec{r}}+ \\
& +K^{2}\left[\sum_{m \neq 0} \chi_{m} e^{i h_{m} x}\right]\left[\sum_{l} e^{i \vec{k}_{l \| l} \vec{e}_{l}} E_{l}(z)\right]=0
\end{aligned}
$$

To eliminate the exponents, we make use of the phase-shift property of the Fourier series, given by:

$$
f(x)=\sum_{a} c_{a} e^{i a x}=\sum_{a-b} c_{a-b} e^{i(a-b) x} .
$$

We shift the expansion of $\chi$ by $-l$ :

$$
\begin{aligned}
& \sum_{m}\left(\kappa_{z m}^{2}+\frac{d^{2}}{d z^{2}}\right) \vec{e}_{m} E_{m}(z) e^{i \vec{k}_{m \| \mid} \cdot \vec{r}}+ \\
&+K^{2}\left[\sum_{m \neq l} \chi_{m-l} e^{i\left(h_{m}-h_{l}\right) x}\right]\left[\sum_{l} e^{i \vec{K}_{||} \vec{r}+h_{l} x} \vec{e}_{l} E_{l}(z)\right]=0,
\end{aligned}
$$




$$
\begin{aligned}
& {\left[\sum_{m}\left(\kappa_{z m}^{2}+\frac{d^{2}}{d z^{2}}\right) \vec{e}_{m} E_{m}(z)+\right.} \\
& \left.\quad+K^{2}\left(\sum_{m \neq l} \chi_{m-l}\right)\left(\sum_{l} \vec{e}_{l} E_{l}(z)\right)\right] e^{i \vec{k}_{m \|} \cdot \vec{r}}=0 .
\end{aligned}
$$

With all the exponential functions equal in one order, we must solve the wave equation for each order separately due to the orthogonality of the plane waves, resulting in the infinite set of equations:

$$
\left(\kappa_{z m}^{2}+\frac{d^{2}}{d z^{2}}\right) \vec{e}_{m} E_{m}(z)+K^{2} \sum_{l \neq m} \chi_{m-l} \vec{e}_{l} E_{l}(z)=0 .
$$

We search for a solution of system Eq. 6.16 in the form of a monochromatic standing plane wave:

$$
E_{m}(z)=\sum_{n}\left[T_{n} e^{i k_{z n}\left(z-z_{j}\right)}+R_{n} e^{-i k_{z n}\left(z-z_{j}\right)}\right] E_{m n}
$$

The $\pm k_{z n}$ wave modes are included because Eq. 6.17 is invariant with respect to the sign of $k_{z n}$, and $T_{n}$ and $R_{n}$ have the physical meaning of complex amplitudes of transmitted (downward travelling) and reflected (upward travelling) waves. The phase is set to be zero at the bottom interface of the layer. Again, we must solve the above equations for each wave mode independently due to the exponentials which can be divided out of the equations. We obtain the final set of equations by multiplying with $\vec{e}_{m}$, to reduce the vector equations to scalar equations (for each $m, n)$ :

$$
\left(\kappa_{z m}^{2}-k_{z n}^{2}\right) E_{m n}+\sum_{l \neq m} \chi_{m-l} E_{l n} \vec{e}_{m} \cdot \vec{e}_{l}=0
$$

These sets of equations describe an eigenvalue problem with eigenvalues $k_{z n}^{2}$ and eigenvectors $\vec{E}_{n}$ :

$$
\mathbf{A}=\left(\begin{array}{ccccc}
\ddots & {\left[\mathbf{A}-k_{z n}^{2} \mathbf{I}\right] \vec{E}_{n}=\overrightarrow{0}} & \\
& \kappa_{-1 z}^{2} & K^{2} \chi_{-1} & K^{2} \chi_{-2} & \\
& K^{2} \chi_{1} & \kappa_{0 z} & K^{2} \chi_{-1} & \\
& K^{2} \chi_{2} & K^{2} \chi_{1} & \kappa_{1 z}^{2} & \\
& & & & \ddots
\end{array}\right)
$$

The number of solutions (eigenvalue/vector pairs) is infinite, but the wave modes corresponding to high diffraction orders ( $m$ far from zero) are in practice weak. This allows us to limit the number of modes included to $\mathcal{D}$, so that numerical evaluation of the eigenvalue problem Eq. 6.20 becomes possible. The formula for the field in a 
structured layer is then given by:

$$
\begin{aligned}
\vec{E}(\vec{r})= & \sum_{m} \sum_{n}\left[T_{n} e^{i k_{z n} z}+R_{n} e^{-i k_{z n} z}\right] . \\
& \vec{e}_{m} E_{m n} e^{i\left(h_{m}+K_{x}\right) x} e^{i K_{y} y},
\end{aligned}
$$

$k_{z n}$ and $E_{m n}$ are determined by numerical solving of the eigenvalue problem, $R_{n}$ and $T_{n}$ by application of the boundary conditions.

\subsection{Boundary conditions}

The boundary conditions on the electric field require that at every interface the parallel component of the electric field is continuous (as can be derived from Maxwell's equations) [25]. In order to apply these boundary conditions, we use the following definitions. We define the matrix $\mathbf{k}$ as the diagonal matrix of eigenvalues $k_{z n}$ and $\mathbf{E}$ as the matrix of column eigenvectors corresponding to it. $\vec{T}$ and $\vec{R}$ denote column vectors containing the coefficients $T_{n}, R_{n} . \vec{T}$ and $\vec{R}$ are the complex-valued amplitudes of the electric field wave modes described by $\mathbf{k}$ and $\mathbf{E}$ that together form the standing wave introduced in Eq. 6.17. In order to apply the boundary conditions across structured and homogeneous layers in the same formulation, we define for homogeneous layers $\mathbf{k}$ as containing the spherical dispersion components $\kappa_{m z}$ on the diagonal and $\mathbf{E}$ as the identity matrix $\mathbf{I}$. These quantities have the shapes $\mathbf{k}, \mathbf{E}$ $\epsilon \mathbb{C}^{\mathcal{D} \times \mathcal{D}}$ and $\vec{T}, \vec{R} \in \mathbb{C}^{\mathcal{D}}$.

As the boundary conditions link these quantities across different layers $j$, the notation $\mathcal{A}^{(j)}$ denotes quantity $\mathcal{A}$ in layer $j$, and $\mathcal{A}_{j-1, j}=\left(\mathcal{A}^{(j-1)}\right)^{-1} \mathcal{A}^{(j)}$.

The boundary conditions require continuity of the parallel components of the field and its normal $(z)$ derivative at every $\left(x, y, z_{j}\right)$ at interface $j$ between layer $j-1$ and $j$. The occurrence of the term $e^{i\left(\left(K_{x}+h_{m}\right) x+K_{y} y\right)}$ requires these equations to be solved for each diffraction order $m$ separately. The field at the bottom of a layer, per order $m$, is given by $\left(\begin{array}{ll}\mathbf{E} & \mathbf{E}\end{array}\right)\left(\begin{array}{l}\vec{T} \\ \vec{R}\end{array}\right)$ and its $z$ derivative by $\left(\begin{array}{ll}\mathbf{k E} & -\mathbf{k E}\end{array}\right)\left(\begin{array}{l}\vec{T} \\ \vec{R}\end{array}\right)$. Both continuity conditions can be combined into one matrix equation by defining the boundary matrix:

$$
\mathbf{P}^{(j)}=\left(\begin{array}{cc}
\mathbf{E}^{(j)} & \mathbf{E}^{(j)} \\
\mathbf{k}^{(j)} \mathbf{E}^{(j)} & -\mathbf{k}^{(j)} \mathbf{E}^{(j)}
\end{array}\right),
$$

and propagation matrix $\mathbf{Q}^{(j)}$ :

$$
\mathbf{Q}^{(j)}=\left(\begin{array}{cc}
\mathbf{Q}^{(j)+} & 0 \\
0 & \mathbf{Q}^{(j)-}
\end{array}\right) .
$$

With $\mathbf{Q}^{(j) \pm}$ the diagonal matrix with elements

$$
Q_{n n}=e^{\mp i \mathbf{k}_{n n}^{(j)} t_{j}}
$$

so that the boundary conditions can be written as

$$
\mathbf{P}^{(j-1)}\left(\begin{array}{l}
\vec{T}^{(j-1)} \\
\vec{R}^{(j-1)}
\end{array}\right)=\mathbf{P}^{(j)} \mathbf{Q}^{(j)}\left(\begin{array}{l}
\vec{T}^{(j)} \\
\vec{R}^{(j)}
\end{array}\right) .
$$


Or equivalently

$$
\left(\begin{array}{l}
\vec{T}^{(j-1)} \\
\vec{R}^{(j-1)}
\end{array}\right)=\mathbf{N}^{(j)}\left(\begin{array}{l}
\vec{T}^{(j)} \\
\vec{R}^{(j)}
\end{array}\right) ; \quad \mathbf{N}^{(j)} \equiv \mathbf{P}_{j-1, j} \mathbf{Q}^{(j)},
$$

where $\mathbf{N}^{(j)}$ is the transfer matrix of layer $j$.

Notice that here we have linked all the wave modes of order $m$ in layer $j$ to all the wave modes of order $m$ in layer $j-1$ using scalar addition of the entire field. As in s polarization the field is parallel to the surface, there is no distinction between parallel component and the complete field. And due to the specific geometry here, the fields of one order of diffraction $m$ all lie in the same direction so scalar addition is in this case equal to vector addition. So while it looks like the field is treated as scalar at this point, this is also the exact solution for the vector treatment.

For the multilayer as a whole we define the transfer matrix as

$$
\mathbf{M}=\left(\begin{array}{ll}
\mathbf{M}_{11} & \mathbf{M}_{12} \\
\mathbf{M}_{21} & \mathbf{M}_{22}
\end{array}\right)=\prod_{j=1}^{N+1} \mathbf{N}^{(j)}
$$

This matrix couples the vacuum and substrate wave fields:

$$
\left(\begin{array}{l}
\vec{T}^{(0)} \\
\vec{R}^{(0)}
\end{array}\right)=\mathbf{M}\left(\begin{array}{l}
\vec{T}^{(N+1)} \\
\vec{R}^{(N+1)}
\end{array}\right) .
$$

In order to solve this system, two boundary conditions are applied to the vacuum and substrate layers:

1. The incident beam is the planar wave of order $m=0$, so that $\vec{T}^{(0)}=$ $(0, \ldots, 0,1,0, \ldots, 0)$.

2. The substrate has no lower interface as it is semi-infinite, so reflected waves are not excited there: $\vec{R}^{(N+1)}=\overrightarrow{0}$.

\subsection{Reflection}

Based on the boundary conditions, the reflected waves have amplitudes

$$
\vec{R}^{(0)}=\mathbf{M}_{21}\left(\mathbf{M}_{11}\right)^{-1} \vec{T}^{(0)},
$$

and the intensity reflected to order $m$ is $\left|R_{m}^{(0)}\right|^{2}$. The diffraction efficiency - the power per unit beam area relative to the incident beam - is then

$$
\mathcal{R}_{m}=\left|R_{m}^{(0)}\right|^{2} \frac{\kappa_{m z}}{\kappa_{0 z}} .
$$

\subsection{E Near field}

In order to calculate the near field using Eq. 6.21 it is necessary to calculate $\vec{T}^{(j)}$, $\vec{R}^{(j)}$ for all layers $j . \vec{T}^{(N+1)}$ is given by:

$$
\vec{T}^{(N+1)}=\left(\mathbf{M}_{11}\right)^{-1} \vec{T}^{(0)}
$$


and then Eq. 6.26 is used to solve for $\vec{T}^{(j)}, \vec{R}^{(j)}$ in the $N$ layers between substrate and vacuum.

Efficient calculation of the near field is done using a linear algebra formulation of Eq. 6.21:

$$
E_{x}(x, y, z)=Y(y) \vec{X}(x)\left(\mathbf{e}_{x} \circ \mathbf{E} \quad \mathbf{e}_{x} \circ \mathbf{E}\right) \mathbf{Z}(z)\left(\begin{array}{c}
\vec{T} \\
\vec{R}
\end{array}\right)
$$

where

$$
\begin{gathered}
Y(y)=e^{i K_{y} y} \\
\vec{X}(x)=\left(\ldots, e^{i\left(K_{x}+h_{-1}\right) x}, e^{i\left(K_{x}+h_{0}\right) x}, e^{i\left(K_{x}+h_{1}\right) x}, \ldots\right), \\
\mathbf{Z}=\left(\begin{array}{cc}
\mathbf{Z}^{+}(z) & 0 \\
0 & \mathbf{Z}^{-}(z)
\end{array}\right), Z_{n n}^{ \pm}(z)=e^{ \pm i k_{n n}\left(z-z_{j}\right)}
\end{gathered}
$$

$\mathbf{Z}^{ \pm}$is diagonal, $\mathbf{e}_{x}$ is the matrix containing the $x$ components of the vectors $\vec{e}_{m n}$ and $\circ$ is the elementwise (Hadamard) multiplication operator, so that $(\mathbf{e} \circ \mathbf{E})_{i j}=\mathbf{e}_{i j} \mathbf{E}_{i j}$. 


\section{References}

[1] R. S. Patti, Three-dimensional integrated circuits and the future of system-onchip designs, Proceedings of the IEEE 94, 1214 (2006).

[2] J.-G. Zhu, Y. Zheng, and G. A. Prinz, Ultrahigh density vertical magnetoresistive random access memory (invited), Journal of Applied Physics 87, 6668 (2000).

[3] B. Black, D. W. Nelson, C. Webb, and N. Samra, 3D processing technology and its impact on iA32 microprocessors, in IEEE International Conference on Computer Design: VLSI in Computers and Processors, 2004. ICCD 2004. Proceedings. (2004) pp. 316-318.

[4] S. Goh, H. Bastiaens, B. Vratzov, Q. Huang, F. Bijkerk, and K. Boller, Fabrication and characterization of free-standing, high-line-density transmission gratings for the vacuum UV to soft X-ray range, Opt. Express 23, 4421 (2015).

[5] B. Beckhoff, R. Fliegauf, M. Kolbe, M. Müller, J. Weser, and G. Ulm, Reference-free total reflection X-ray fluorescence analysis of semiconductor surfaces with synchrotron radiation, Analytical Chemistry 79, 7873 (2007).

[6] D. K. Bowen and B. K. Tanner, X-ray metrology in semiconductor manufacturing (CRC Press, 2006).

[7] R. L. Jones, T. Hu, E. K. Lin, W.-L. Wu, R. Kolb, D. M. Casa, P. J. Bolton, and G. G. Barclay, Small angle X-ray scattering for sub-100 nm pattern characterization, Applied Physics Letters 83, 4059 (2003).

[8] J. Pomplun, S. Burger, L. Zschiedrich, and F. Schmidt, Adaptive finite element method for simulation of optical nano structures, physica status solidi (b) 244, 3419 (2007).

[9] V. Soltwisch, P. Hönicke, Y. Kayser, J. Eilbracht, J. Probst, F. Scholze, and B. Beckhoff, Element sensitive reconstruction of nanostructured surfaces with finite elements and grazing incidence soft X-ray fluorescence, Nanoscale 10, 6177 (2018).

[10] J. Butters, Progress in optics volume XVII, Optics Laser Technology 13, 108 (1981).

[11] M. Nevière, Bragg-Fresnel multilayer gratings: electromagnetic theory, J. Opt. Soc. Am. A 11, 1835 (1994).

[12] M. G. Moharam and T. K. Gaylord, Rigorous coupled-wave analysis of grating diffraction - e-mode polarization and losses, J. Opt. Soc. Am. 73, 451 (1983).

[13] P. Mikulík and T. Baumbach, X-ray reflection by rough multilayer gratings: Dynamical and kinematical scattering, Phys. Rev. B 59, 7632 (1999). 
[14] A. Y. Gaevskii, I. E. Golentus, and V. B. Molodkin, Grazing-incidence dynamic $X$-ray diffraction from a crystal with a shaped surface, Crystallography Reports 57, 708 (2012).

[15] H. Archibald, W. M. John, and M. N. Francis, Diffraction contrast of electron microscope images of crystal lattice defects-II. The development of a dynamical theory, Proceedings of the Royal Society of London. Series A. Mathematical and Physical Sciences 263, 217 (1961).

[16] B. W. Batterman and H. Cole, Dynamical diffraction of X-rays by perfect crystals, Rev. Mod. Phys. 36, 681 (1964).

[17] S. T. Peng, T. Tamir, and H. L. Bertoni, Theory of periodic dielect waveguides, IEEE Transactions on Microwave Theory and Techniques 23, 123 (1975).

[18] V. Soltwisch, A. Fernández Herrero, M. Pflüger, A. Haase, J. Probst, C. Laubis, M. Krumrey, and F. Scholze, Reconstructing detailed line profiles of lamellar gratings from GISAXS patterns with a Maxwell solver, Journal of Applied Crystallography 50, 1524 (2017).

[19] V. Soltwisch, C. Laubis, A. F. Herrero, M. Pflüger, A. Haase, and F. Scholze, Investigating surface structures by EUV scattering, Proc.SPIE 10143, 10143 (2017).

[20] M. Pisarenco, R. Quintanilha, M. G. M. M. van Kraaij, and W. M. J. Coene, Fast semi-analytical solution of Maxwell's equations in Born approximation for periodic structures, J. Opt. Soc. Am. A 33, 610 (2016).

[21] A. M. Afanas'ev and M. K. Melkonyan, X-ray diffraction under specular reflection conditions. Ideal crystals, Acta Crystallographica Section A 39, 207 (1983).

[22] V. M. Kaganer, V. L. Indenbom, M. Vrána, and B. Chalupa, Laue-to-Bragg transition in extremely asymmetric dynamic neutron diffraction, physica status solidi (a) 71, 371 (1982).

[23] P. G. Medvedev, A. M. Afanas'ev, and M. A. Chuev, Anomalous Kossel effect in semiconductor structures, Russian Microelectronics 35, 359 (2006).

[24] J. J. Hench and Z. Strakoš, The RCWA method - a case study with open questions and perspectives of algebraic computations, Vol. 31 (Institute of Computational Mathematics, 2008) pp. 331-357.

[25] M. Born and E. Wolf, Principles of optics: electromagnetic theory of propagation, interference and diffraction of light (Elsevier, 2013). 



\section{VALORIZATION AND OUTLOOK}

Ultra-thin multilayers, single-crystal surfaces and 3D patterned nanoscale devices, all require state-of-the-art nanometrology to establish the basic structure parameters. The research summarized in this thesis can be described as a theoretical and numerical study of X-ray scattering in a broad sense. It was motivated to advance the current state of several X-ray analysis methods, such as X-ray reflectivity (XRR), X-ray diffraction (XRD), X-ray standing wave (XSW) and grazing incidence small angle X-ray scattering (GISAXS) to a new level in accuracy for the study of ultra-thin multilayers, single-crystal surfaces and 3D patterned nanoscale devices.

The combined analysis of X-ray and EUV measurements, as developed and described in this thesis, allowed us to drastically improve the precision of characterization of the multilayer mirrors. This has been cited in the work of Haase et.al. [1] where another step in the improvement of the characterization was done by incorporating resonant EUV reflectivity, X-ray standing wave and X-ray reflectivity data, all within one mathematical model. The next step was made in the research of Tryus et.al. [2], where a lab-scale tool for EUV reflectivity measurements was developed, while in our research EUV reflectivity measurements were done using a synchrotron. In this research our combined analysis approach has been successfully employed.

We have demonstrated an approach for the analysis of the $3 \mathrm{D}$ distribution of density fluctuations in one of the materials of a multilayer mirror using grazingincidence small-angle X-ray scattering. We observed that the formation of structural imperfections in the bulk layer affects the interface roughness morphology in the multilayer. That observation is of particular interest, since the morphology of interface roughness drastically affects the total performance of the multilayer mirrors. Thus, further refinement of multilayer mirrors may require special attention not only to interfaces but also to $3 \mathrm{D}$ bulk defects in layers. The demonstrated approach can be used for a detailed study of such defects.

Moving from the basically $1 \mathrm{D}$ periodical multilayer structures to $2 \mathrm{D}$ and $3 \mathrm{D}$ nanoscale structures such as gratings and nano-columns, we have developed a mathematical model which allows to selectively analyze the elemental distribution in the structures. This approach enabled the analysis of the 3D elemental distribution in periodic nanoscale structures for the first time. It can be useful for a broad spectrum of nano-scale devices like light-trapping structures in solar cells, field emitter arrays, nanorods, devices for surface-enhanced Raman scattering and surface-enhanced infrared absorption spectroscopy.

A practical feature of the research is that many of the proposed nano-metrology 
methods can be applied on lab-scale equipment. For instance, with the development of lab-based plasma EUV sources, combined X-ray and EUV reflectivity analysis can be applied. With modern X-ray diffractometer instruments, like the Malvern Panalytical Empyrean, the bulk distribution of defects in multilayers can be studied using our approach developed for the density-fluctuation study. Also, in our theoretical study of grazing-incidence X-ray diffraction we showed that specular reflectivity modulated by diffraction can be used for the study of the crystal surface structure.

\section{References}

[1] A. Haase, S. Bajt, P. Hönicke, V. Soltwisch, and F. Scholze, Multiparameter characterization of subnanometer $\mathrm{Cr} / \mathrm{Sc}$ multilayers based on complementary measurements, Journal of Applied Crystallography 49, 2161 (2016).

[2] M. Tryus, Extreme ultraviolet reflectometry for structural and optical characterization of thin films and layer systems, Ph.D. thesis, RWTH Aachen University (2018). 


\section{SUMMARY}

The research described in this thesis concerns X-ray and Extreme UV characterization of periodic nanoscale structures. Advances in this analysis range from characterization of 1D systems, like multilayer short-wavelength mirrors, to nanoscale systems with a $2 \mathrm{D}$ or $3 \mathrm{D}$, complex architecture. We have drastically improved the accuracy of the multilayer structure characterization by combining the analysis of $\mathrm{X}$-ray reflectivity data and EUV normal-incidence reflectivity data, as compared to the traditional, sole use of X-ray analysis. This was done by using an uncertainty analysis based on the covariation matrix of the combined goodness of fit criterion for data taken at both wavelengths. It shows that the X-ray data primarily contribute to the accuracy of the layer and interface thicknesses, while the EUV data are more sensitive to the layer densities and atomic compositions.

Multilayer mirrors can have imperfections distributed in all three dimensions. In a $\mathrm{W} / \mathrm{Si}$ multilayer system for instance, a $3 \mathrm{D}$ distribution of density fluctuations was observed in the Si layers. These fluctuations have been studied using grazingincidence small-angle X-ray scattering. Assuming a 3D para-crystal-like fluctuation of the density distribution, the parameters reconstructed by numeric simulations were found to be in excellent agreement with independent observations by scanning transmission electron microscopy. Moreover, the density fluctuations were noted to affect the morphology of the interface roughness.

In subsequent theoretical research using grazing-incidence X-ray diffraction, the surface structure of monocrystals was further assessed. The structures were considered as $2 \mathrm{D}$ objects, assessed by probing the distribution of atoms in depth of the sample and in a chosen set of planes parallel to the surface. Using advances in the dynamical diffraction theory, we predicted an interesting effect: a signature of the off-plane diffraction can be observed by measuring the specular reflectivity. Another interesting aspect is that the intensity of the specular reflection is significantly higher than that of the diffracted beam. This may allow the characterization of the crystal surface, the surface oxidation and the thin (epitaxial) layers, using a relatively moderate power, lab-scale X-ray metrology tool as opposed to a synchrotron-based facility conventionally used for these purposes.

The dimensionality was also addressed in the $\mathrm{X}$-ray standing wave technique. Although it allows the analysis of the atomic in-depth distribution of different species, information on the lateral direction is normally lost in this approach. We have derived equations based on the dynamical diffraction theory in a many-beam approximation, which allows the detailed analysis of the 3D atomic distribution in the structure. This has been verified on experimental data measured on a $\mathrm{Si}_{3} \mathrm{~N}_{4}$ lamellar grating structure using an incident photon energy in the EUV range and on a 3D nano-column $\mathrm{Cr}$ structure in the hard X-ray range. The result of simulations using our mathematical model showed a good agreement with nominal structure parameters. 
In conclusion, this thesis includes several analytical approaches to extend the scope of current X-ray and EUV analysis. This notably concerns the broadening of the dimensionality of the analysis of nano-scale devices and structures, and methods to arrive at an enhanced precision by combining data from different wavelength ranges. 


\section{SAMENVATTING}

Het onderzoek in dit proefschrift gaat over de karakterisatie van periodieke structuren op nanoschaal met behulp van röntgen (X-ray) en Extreem UV straling. Er wordt vooruitgang beschreven in het karakteriseren van 1D systemen zoals multilaagspiegels voor korte golflengtes, en nanoschaal objecten met een complexe $2 \mathrm{D}$ of 3D structuur. De nauwkeurigheid van de analyse van multilaagstructuren is enorm verbeterd door de gecombineerde analyse van harde X-ray en EUV reflectiviteitsdata, vergeleken met de traditionele analysemethode die alleen op X-ray data gebaseerd is. Dit is bereikt door middel van een nauwkeurigheidsanalyse die gebruikt maakt van de covariantiematrix van de fitfunctie die gebaseerd is op data voor beide golflengtes. Hieruit blijkt dat de harde X-rays voornamelijk bijdragen aan de bepaling van de laag- en interfacediktes, terwijl de EUV data juist veel gevoeliger zijn voor laagdichtheden en materiaalcomposities.

Multilaagspiegels kunnen structurele onvolmaaktheden hebben in alle drie dimensies. In een $\mathrm{W} / \mathrm{Si}$ multilaagsysteem zijn bij voorbeeld driedimensionale variaties van elektronendichtheden waargenomen in de Si lagen. Deze variaties zijn bestudeerd met behulp van X-ray verstrooiing bij kleine invalshoeken. Uitgaande van een $3 \mathrm{D}$ parakristal-achtige verdeling van de dichtheidsvariaties, was het mogelijk om een zeer goede overeenkomst te behalen tussen gereconstrueerde structuur parameters uit de X-ray data, en de structuurparameters die uit een scanning transmissie-elektronenmicroscoop analyse verkregen zijn. Verder is ook gebleken dat de dichtheidsvariaties in de Si lagen een directe invloed hebben op de morfologie van de interfaces tussen de lagen.

Het doen van X-ray diffractie metingen bij kleine invalshoek is theoretisch geevalueerd om de structuur dichtbij het oppervlak van periodieke structuren zoals kristallen te analyseren. De structuren werden benaderd als tweedimensionale objecten, en de diepteverdeling van atomen in een set van vlakken die parallel aan het oppervlak liggen werd beschouwd. Door middel van vooruitgang in de theorie van dynamische diffractie werd een interessant effect voorspeld: als gevolg van diffractie aan vlakken niet parallel aan het oppervlak, wordt het reflectiesignaal van de structuur sterk beïnvloedt. Doordat dit reflectiesignaal veel sterker is dan het diffractiesignaal, zorgt deze ontdekking ervoor dat de karakterisatie van dit soort kristaloppervlakken gedaan kan worden met een lage intensiteit X-ray bron zoals in een laboratoriumdiffractieapparaat, in tegenstelling tot het tot nu toe noodzakelijke gebruik van een moeilijk toegankelijke hoge intensiteit X-ray bron zoals een synchrotron.

De dimensionaliteit van periodieke structuren is ook benaderd door middel van het gebruik van staande X-ray golven. Hoewel staande X-ray golven gebruikt kunnen worden om de in-diepte verdeling van elementen in een structuur te onderzoeken, gaat hierbij normaal de laterale informatie verloren. In dit proefschrift zijn vergelijkingen afgeleid voor dynamische diffractie in de meerdere-bundel benadering, 
waardoor de laterale informatie niet verloren gaat, wat leidt tot de mogelijkheid om met staande X-ray golven een analyse te doen van de driedimensionale verdeling van elementen in een structuur. Deze theorie is getoetst door middel van experimentele EUV data van een $\mathrm{Si}_{3} \mathrm{~N}_{4}$ laminaire tralie, en door middel van harde X-ray data van een driedimensionale nanokolomnaire $\mathrm{Cr}$ structuur. De resultaten van de modelberekeningen komen goed overeen met de experimentele data, en de structuurparameters die uit deze analyse voortkomen zijn in goede overeenstemming met de nominale structuurparameters en parameters uit eerdere analyse.

Samengevat, dit proefschrift bevat meerdere analytische benaderingen die de huidige mogelijkheden van X-ray en EUV analyse verder uitbreiden. Specifiek richt het zich op het uitbreiden van de mogelijkheden om structuren met hoge dimensionaliteit te bestuderen, en op het verbeteren van de nauwkeurigheid waarmee de structuur onderzocht wordt door middel van combineren van data uit verschillende golflengtegebieden. 


\section{ЗАКЛЮЧЕНИЕ}

В этой диссертации исследуются методы анализа периодических наноразмерных структур при помощи излучения в рентгеновском диапазоне и в диапазоне глубокого ультрафиолета (УФ). В работе рассматривается как анализ одномерных структур, таких как многослойные коротковолновые зеркала, так и анализ структур со сложной двухмерной или трёхмерной архитектурой. Совместив анализ данных рентгеновской рефлектометрии в скользящем падении с анализом данных УФ рефлектометрии в нормальном падении в рамках одной математической модели, мы значительно улучшили точность характеризации многослойных структур. Улучшение точности характеризации было продемонстрировано с помощью анализа, основанного на ковариационных матрицах рассчитанных для данных в рентгеновском и в УФ диапазонах. Этот анализ показал, что данные рентгеновской рефлектометрии в основном чувствительны к толщинам слоёв и к характерным толщинам взаимной диффузии слоёв, а данные УФ рефлектометрии чувствительны к плотностям материалов и стехиометрии элементного состава материалов структуры.

Многослойные дифракционные зеркала, по своей сути, являются одномерными структурами, однако дефекты таких структур могут иметь трёхмерный характер. Трёхмерное распределение флуктуаций плотности было обнаружено в слоях кремния многослойной системы W/Si. Эти флуктуации были изучены с использованием малоуглового рентгеновского рассеяния в скользящем падении. Статистические параметры распределения флуктуаций плотности были получены при помощи численного моделирования экспериментальных данных. Математическая модель флцктуаций была построена исходя из предположения, что флуктации распределенны как трёхмерный пара-кристалл. Полученные таким образом, статистические параметры распределения флуктаций плотности хорошо согласуются с экспериментальными данными просвечивающей электронной микроскопии. Более того, было замечено, что флуктации плотности влияют на морфологию шероховатости границ раздела многослойной структуры.

Далее, в теоретическом исследовании в рамках рентгеновской дифракции в скользящем падении была рассмотрена структура идеальных монокристаллов. Структуры были рассмотрены как двухмерные объекты: атомы распределены по глубине образца и в выбранном наборе кристаллографических плоскостей, параллельных поверхности. С помощью динамической теории дифракции был теоретически предсказан интересный эффект: интенсивность зеркально отражённого рентгеновского излучения модулируется осцилляциями интенсивности дифракции вне плоскости падения. Учитывая то, что интенсивность отражённого луча в условиях скользящего падения под углом, близким к углу полного внешнего отражения, значительно выше интенсивности дифракции вне плоскости падения. Это может позволить анализировать структуры поверхности 
кристаллов, приповерхностных слоёв оксидов и тонких эпитаксиальных плёнок, используя лабораторный дифрактометр на рентгеновской трубке, мощность которой значительно ниже мощности синхротрона или лазера на свободных электронах.

Проблема размерности была также рассмотрена в применении к методу стоячих рентгеновских волн (СРВ). Классический метод СРВ позволяет раздельно анализировать распределения концентрации различных элементов в структуре по глубине. В таких исследованиях информация о латеральном распределении концентрации утрачивается. Основываясь на динамической теории дифракции в многоволновом приближении, мы вывели уравнения, учитывающие трёхмерные распределения концентрации различных элементов в структуре. Полученная теория была проверена экспериментально для УФ диапазона на образце нитрид кремниевой наноразмерной дифракционной решётки и для рентгеновского диапазона на образце трёхмерных нано-столбцов хрома. Результат численного моделирования хорошо согласуется с экспериментальными данными.

В заключение, набор аналитических подходов, описанный в этой диссертации, позволяет расширить область применения современных методов рентгеноструктурного анализа и улучшить качество анализа наноразмерных структур. В частности, описанные подходы могут быть применены в области анализа трёхмерных наноразмерных структур, как в рентгеновском, так и в УФ диапазонах. 


\section{ACKNOWLEDGMENTS}

I was favoured to meet many people who had helped me and guided me during the course of this PhD study. I am gratefull to prof. Fred Bijkerk for giving me an oportunity to become a $\mathrm{PhD}$ in the XUV optics group and for the exceptionally professional and friendly atmosphere I have been working and learning in these four and a half years. I am thankful to my supervisor dr. Igor Makhotkin with whom we attacked the scientific problems side by side and to dr. Robbert van de Kruijs who had helped me to scale up the rockiest part of this project. I would like to especially acknowledge dr. Sergey Yakunin. He introduced me to the real science ten years ago, stayed in touch and adviced me in many different projects despite the distance, for which I am very greatfull. I am indebted to prof. Mikhail Chuev for his highly educated advice and guidance. I am also grateful to Carin Krijnen and Jacqueline Emmerich-Nijenhuis for helping me navigate through administration and to dr. Eric Louis for wise words of advice.

It would have been impossible to complete this $\mathrm{PhD}$ project without my large, friendly and supportive family. Especially my dear wife Elena Kostkina, whose love is proven to be space-time invariant. Thank you very much for supporting me and helping to endure, my dear! Huge thanks to my parents Vladimir Nikolaev and Nadezhda Nikolaeva for suporting in my determination to become a scientist, to my darling sister Elena Boguslavskaya for participation and advice and to my endearing nephew and niece Dmitry Boguslavsky and Valeria Boguslavskaya for the inspiration as well as to my brothers in law Georgy Bogulavsky and Alexey Kostkin. I am also grateful to my old friends Roman Zuev, Igor Shaposhnikov and Alexander Rodionov who have been with me since childhood and virtually become a part of the family.

I also want to acknowledge my colleagues and friends with whom I spend time at work and in leisure. Huge thanks to dr. Andrey Zameshin whose big and broad set of knowledge made our discussions interesting and engaging. Also to Parikshit Phadke for his warm support practically in any aspect of $\mathrm{PhD}$ life. To dr. Dmitry Kuznetsov for openness and strong friendship as well as to Igor Milov for strong partnership in science and friendship in life. For warmly welcoming me as their friend I am very grateful to Lucas Kostek and Cristiane Stilhano Vilas Boas.

I was favored to have good comrades with whom we worked together on various projects: Joris de la Rie, Roman Medvedev, Airat Shafikov, Olena Soroka, Oleksandr Antonov and Cedric Hendrikx. And especially my good friend and comrade dr. Maksim Tryus who is ready to fight for the nice results till accomplish. Huge thanks to my good friend Johan Reinink for helping me navigate in Dutch culture and in experimental aspects of physics. For keeping warm friendship despite the distance I would like to acknowledge my good friends and colleagues from Kurchatov institute Alexander Rogachev and Grigory Prutskov as well as Ilia Subbotin, Igor Likhachev and Sergey Tihomirov. I would also like to thank my friend 
from the MIPT, dr. Sergey Soloviev, whom I consider to be a role model, as a peer theoretical physicist of modern times. I am also thankful to my colleagues from PTB dr. Victor Soltwisch, dr. Frank Scholze, dr. Philipp Hönicke and Analia Fernández Herrero for openness and readiness to participate in our research. And I am thankful to my friends who shared leisure time, hobbies and interests with me: Elizaveta Gasyuk, Dmitry Grishkin, Fedor Zorky, Vadim Zaskovets, Maksim Morozov, Zinaida Kostyuchenko and others.

Finally, I want to express my gratitude to my school physics teacher Natalia Ivanovna Komissarova to whom this book is dedicated to. She introduced me to physics, inspired me to study and helped me to find my direction in life. For which I am infinitely obliged, thank you very much! 
ISBN: 978-90-365-4798-7

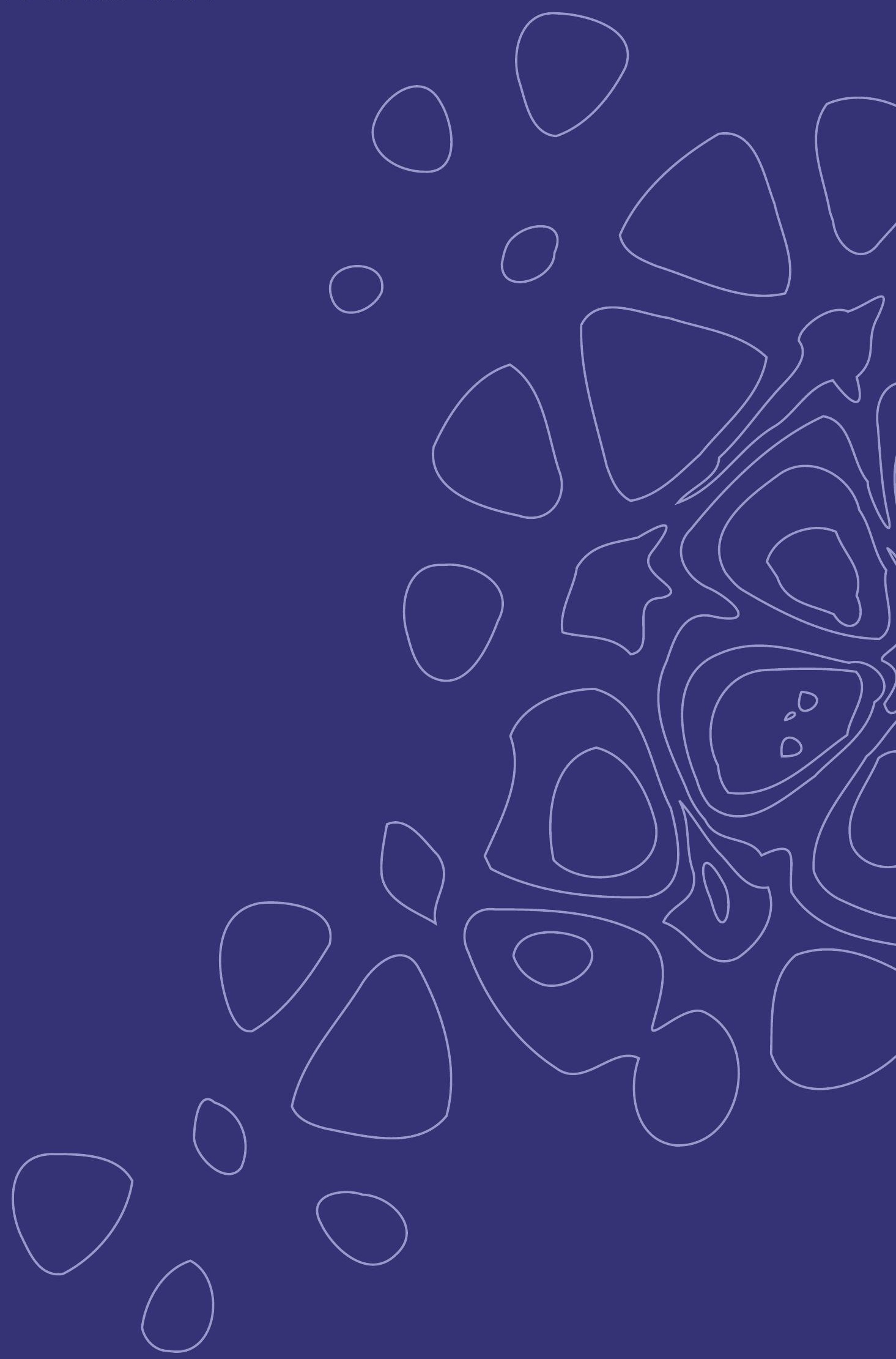

